\title{
Measurement and Interpretation of Fermion-Pair Production at LEP energies from 130 to $172 \mathrm{GeV}$
}

\author{
DELPHI Collaboration
}

\begin{abstract}
The data collected with the DELPHI detector at centre-of-mass energies between 130 and $172 \mathrm{GeV}$, during LEP operation in 1995 and 1996, have been used to determine the hadronic and leptonic cross-sections and leptonic forwardbackward asymmetries. In addition, the cross-section ratios and forwardbackward asymmetries for flavour-tagged samples of light (uds), c and b quarks have been measured.

The results are interpreted by performing S-matrix fits to these data and to the data collected previously at the energies near the $Z^{0}$ resonance peak (88$93 \mathrm{GeV})$. The results are also interpreted in terms of contact interactions, which parameterise physics beyond the Standard Model. Further interpretation of the data is made in terms of possible R-parity violating SUSY particles and of possible $Z^{\prime}$ bosons. No significant deviations from the Standard Model expectations are found and limits are given for the various interpretations which are made of physics beyond the Standard Model.
\end{abstract}


P.Abreu $^{21}$, W.Adam ${ }^{50}$, T.Adye ${ }^{36}$, P.Adzic ${ }^{11}$, Z.Albrecht ${ }^{17}$, T.Alderweireld ${ }^{2}$, G.D.Alekseev ${ }^{16}$, R.Alemany ${ }^{49}$, T.Allmendinger ${ }^{17}$, P.P.Allport ${ }^{22}$, S.Almehed ${ }^{24}$, U.Amaldi ${ }^{9}$, S.Amato ${ }^{47}$, E.G.Anassontzis ${ }^{3}$, P.Andersson ${ }^{44}$, A.Andreazza ${ }^{9}$, S.Andringa ${ }^{21}$, P.Antilogus ${ }^{25}, \quad$ W-D.Apel ${ }^{17}, \quad$ Y.Arnoud ${ }^{9}, \quad$ B.Asman ${ }^{44}$, J-E.Augustin ${ }^{25}$, A.Augustinus $^{9}$, P.Baillon ${ }^{9}$, P.Bambade ${ }^{19}$, F.Barao ${ }^{21}$, R.Barbier ${ }^{25}$, D.Y.Bardin ${ }^{16}$, G.Barker ${ }^{17}$, A.Baroncelli ${ }^{38}$, M.Battaglia $^{15}$, M.Baubillier ${ }^{23}$, K-H.Becks ${ }^{52}$, M.Begalli ${ }^{6}$, A.Behrmann ${ }^{52}$, P.Beilliere ${ }^{8}$, Yu.Belokopytov ${ }^{9,53}$, A.C.Benvenuti $^{5}$, C.Berat ${ }^{14}$, M.Berggren ${ }^{25}$, D.Bertini ${ }^{25}$, D.Bertrand ${ }^{2}$, M.Besancon ${ }^{39}$, F.Bianchi ${ }^{45}$, M.Bigi $^{45}$, M.S.Bilenky ${ }^{16}$, M-A.Bizouard ${ }^{19}$, D.Bloch ${ }^{10}$, H.M.Blom ${ }^{30}$, M.Bonesini ${ }^{27}$, W.Bonivento ${ }^{27}$, M.Boonekamp ${ }^{39}$, P.S.L.Booth ${ }^{22}$, A.W.Borgland ${ }^{4}$, G.Borisov ${ }^{19}$, C.Bosio $^{41}, \quad$ O.Botner ${ }^{48}, \quad$ E.Boudinov ${ }^{30}$, B.Bouquet $^{19}$, C.Bourdarios $^{19}$, T.J.V.Bowcock ${ }^{22}$, I.Boyko ${ }^{16}$, I.Bozovic $^{11}, \quad$ M.Bozzo ${ }^{13}, \quad$ P.Branchini ${ }^{38}$, T.Brenke ${ }^{52}$, R.A.Brenner ${ }^{48}$, P.Bruckman ${ }^{18}$, J-M.Brunet ${ }^{8}$, L.Bugge ${ }^{32}$, T.Buran ${ }^{32}$, T.Burgsmueller ${ }^{52}$, P.Buschmann ${ }^{52}$, S.Cabrera $^{49}$, M.Caccia ${ }^{27}$, M.Calvi ${ }^{27}$, T.Camporesi ${ }^{9}$, V.Canale ${ }^{37}$, F.Carena ${ }^{9}$, L.Carroll ${ }^{22}$, C.Caso $^{13}$, M.V.Castillo Gimenez ${ }^{49}$, A.Cattai ${ }^{9}$, F.R.Cavallo ${ }^{5}$, V.Chabaud ${ }^{9}$, N.Chalanda ${ }^{42}$, Ph.Charpentier ${ }^{9}$, L.Chaussard $^{25}$, P.Checchia ${ }^{35}$, G.A.Chelkov ${ }^{16}$, R.Chierici ${ }^{45}$, P.Chliapnikov ${ }^{42}$, P.Chochula ${ }^{7}$, V.Chorowicz ${ }^{25}$, J.Chudoba ${ }^{29}$, K.Cieslik ${ }^{18}$, P.Collins ${ }^{9}$, R.Contri ${ }^{13}$, E.Cortina ${ }^{49}$, G.Cosme ${ }^{19}$, F.Cossutti ${ }^{9}$, J-H.Cowell ${ }^{22}$, H.B.Crawley ${ }^{1}$, D.Crennell ${ }^{36}$, S.Crepe ${ }^{14}$, G.Crosetti ${ }^{13}$, J.Cuevas Maestro ${ }^{33}$, S.Czellar ${ }^{15}$, M.Davenport ${ }^{9}$, W.Da Silva ${ }^{23}$, A.Deghorain ${ }^{2}$, G.Della Ricca ${ }^{46}$, P.Delpierre ${ }^{26}$, N.Demaria ${ }^{9}$, A.De Angelis ${ }^{9}$, W.De Boer ${ }^{17}$, S.De Brabandere ${ }^{2}$, C.De Clercq ${ }^{2}$, B.De Lotto ${ }^{46}$, A.De Min ${ }^{35}$, L.De Paula ${ }^{47}$, H.Dijkstra ${ }^{9}$, L.Di Ciaccio ${ }^{37,9}$, J.Dolbeau $^{8}$, K.Doroba ${ }^{51}$, M.Dracos ${ }^{10}$, J.Drees ${ }^{52}$, M.Dris ${ }^{31}$, A.Duperrin ${ }^{25}$, J-D.Durand ${ }^{9}$, G.Eigen ${ }^{4}$, T.Ekelof ${ }^{48}$, G.Ekspong ${ }^{44}$, M.Ellert ${ }^{48}$, M.Elsing ${ }^{9}$, J-P.Engel ${ }^{10}$, B.Erzen ${ }^{43}$, M.Espirito Santo ${ }^{21}$, E.Falk ${ }^{24}$, G.Fanourakis ${ }^{11}$, D.Fassouliotis ${ }^{11}$, J.Fayot ${ }^{23}$, M.Feindt ${ }^{17}$, A.Fenyuk ${ }^{42}$, P.Ferrari ${ }^{27}$, A.Ferrer ${ }^{49}$, E.Ferrer-Ribas ${ }^{19}$, S.Fichet ${ }^{23}$, A.Firestone ${ }^{1}$, U.Flagmeyer ${ }^{52}$, H.Foeth $^{9}$, E.Fokitis ${ }^{31}$, F.Fontanelli ${ }^{13}$, B.Franek ${ }^{36}$, A.G.Frodesen ${ }^{4}$, R.Fruhwirth ${ }^{50}$, F.Fulda-Quenzer $^{19}$, J.Fuster ${ }^{49}$, A.Galloni ${ }^{22}$, D.Gamba ${ }^{45}$, S.Gamblin ${ }^{19}$, M.Gandelman ${ }^{47}$, C.Garcia ${ }^{49}$, C.Gaspar ${ }^{9}$, M.Gaspar ${ }^{47}$, U.Gasparini ${ }^{35}$, Ph.Gavillet ${ }^{9}$, E.N.Gazis ${ }^{31}$, D.Gele ${ }^{10}$, L.Gerdyukov ${ }^{42}$, N.Ghodbane ${ }^{25}$, I.Gil ${ }^{49}$, F.Glege ${ }^{52}$, R.Gokieli ${ }^{9,51}$, B.Golob ${ }^{43}$, G.Gomez-Ceballos ${ }^{40}$, P.Goncalves ${ }^{21}$, I.Gonzalez Caballero ${ }^{40}$, G.Gopal $^{36}$, L.Gorn ${ }^{1,54}$, M.Gorski ${ }^{51}$, Yu.Gouz ${ }^{42}$, V.Gracco ${ }^{13}$, J.Grahl ${ }^{1}$, E.Graziani ${ }^{38}$, C.Green ${ }^{22}$, H-J.Grimm ${ }^{17}$, P.Gris ${ }^{39}$, G.Grosdidier $^{19}$, K.Grzelak ${ }^{51}$, M.Gunther ${ }^{48}, \quad$ J.Guy $^{36}$, F.Hahn ${ }^{9}$, S.Hahn ${ }^{52}$, S.Haider ${ }^{9}$, A.Hallgren ${ }^{48}$, K.Hamacher $^{52}$, J.Hansen ${ }^{32}$, F.J.Harris ${ }^{34}$, V.Hedberg ${ }^{24}$, S.Heising ${ }^{17}$, J.J.Hernandez ${ }^{49}$, P.Herquet ${ }^{2}$, H.Herr ${ }^{9}$, T.L.Hessing ${ }^{34}$, J.-M.Heuser ${ }^{52}$, E.Higon ${ }^{49}$, S-O.Holmgren ${ }^{44}$, P.J.Holt ${ }^{34}$, S.Hoorelbeke ${ }^{2}$, M.Houlden ${ }^{22}$, J.Hrubec $^{50}$, K.Huet ${ }^{2}$, G.J.Hughes ${ }^{22}$, K.Hultqvist ${ }^{44}$, J.N.Jackson ${ }^{22}$, R.Jacobsson ${ }^{9}$, P.Jalocha ${ }^{9}$, R.Janik ${ }^{7}$, Ch.Jarlskog $^{24}$, G.Jarlskog ${ }^{24}$, P.Jarry ${ }^{39}$, B.Jean-Marie ${ }^{19}$, E.K.Johansson ${ }^{44}$, P.Jonsson ${ }^{25}$, C.Joram ${ }^{9}$, P.Juillot ${ }^{10}$, F.Kapusta $^{23}$, K.Karafasoulis ${ }^{11}$, S.Katsanevas ${ }^{25}$, E.C.Katsoufis ${ }^{31}$, R.Keranen ${ }^{17}$, B.P.Kersevan ${ }^{43}$, B.A.Khomenko ${ }^{16}$, N.N.Khovanski ${ }^{16}$, A.Küskinen ${ }^{15}$, B.King ${ }^{22}$, A.Kinvig ${ }^{22}$, N.J.Kjaer ${ }^{30}$, O.Klapp ${ }^{52}$, H.Klein ${ }^{9}$, P.Kluit $^{30}$, P.Kokkinias ${ }^{11}$, M.Koratzinos ${ }^{9}$, V.Kostioukhine ${ }^{42}$, C.Kourkoumelis ${ }^{3}$, O.Kouznetsov ${ }^{39}$, M.Krammer ${ }^{50}$, E.Kriznic $^{43}$, Z.Krumstein ${ }^{16}$, P.Kubinec ${ }^{7}$, J.Kurowska ${ }^{51}$, K.Kurvinen ${ }^{15}$, J.W.Lamsa ${ }^{1}$, D.W.Lane ${ }^{1}$, P.Langefeld ${ }^{52}$, V.Lapin $^{42}$, J-P.Laugier ${ }^{39}$, R.Lauhakangas ${ }^{15}$, G.Leder ${ }^{50}$, F.Ledroit ${ }^{14}$, V.Lefebure ${ }^{2}$, L.Leinonen ${ }^{44}$, A.Leisos ${ }^{11}$, R.Leitner $^{29}$, J.Lemonne ${ }^{2}$, G.Lenzen ${ }^{52}$, V.Lepeltier ${ }^{19}$, T.Lesiak ${ }^{18}$, M.Lethuillier $^{39}$, J.Libby ${ }^{34}$, D.Liko ${ }^{9}$, A.Lipniacka $^{44}$, I.Lippi ${ }^{35}$, B.Loerstad ${ }^{24}$, M.Lokajicek ${ }^{29}$, J.G.Loken ${ }^{34}$, J.H.Lopes ${ }^{47}$, J.M.Lopez ${ }^{40}$, R.Lopez-Fernandez ${ }^{14}$, D.Loukas ${ }^{11}$, P.Lutz ${ }^{39}$, L.Lyons ${ }^{34}$, J.MacNaughton ${ }^{50}$, J.R.Mahon ${ }^{6}$, A.Maio ${ }^{21}$, A.Malek ${ }^{52}$, T.G.M.Malmgren ${ }^{44}$, V.Malychev ${ }^{16}$, F.Mandl ${ }^{50}$, J.Marco ${ }^{40}$, R.Marco ${ }^{40}$, B.Marechal ${ }^{47}$, M.Margoni $^{35}$, J-C.Marin $^{9}$, C.Mariotti ${ }^{9}$, A.Markou ${ }^{11}$, C.Martinez-Rivero ${ }^{19}$, F.Martinez-Vidal ${ }^{49}$, S.Marti i Garcia ${ }^{9}$, J.Masik ${ }^{12}$, N.Mastroyiannopoulos $^{11}$, F.Matorras ${ }^{40}$, C.Matteuzzi ${ }^{27}$, G.Matthiae ${ }^{37}$, F.Mazzucato ${ }^{35}$, M.Mazzucato ${ }^{35}$, M.Mc Cubbin ${ }^{22}$, R.Mc Kay ${ }^{1}$, R.Mc Nulty ${ }^{22}$, G.Mc Pherson ${ }^{22}$, C.Meroni ${ }^{27}$, W.T.Meyer ${ }^{1}$, E.Migliore ${ }^{45}$, L.Mirabito $^{25}$, W.A.Mitaroff ${ }^{50}$, U.Mjoernmark ${ }^{24}$, T.Moa $^{44}$, M.Moch $^{17}$, R.Moeller $^{28}$, K.Moenig $^{9}$, M.R.Monge $^{13}$, X.Moreau $^{23}$, P.Morettini ${ }^{13}$, G.Morton ${ }^{34}$, U.Mueller ${ }^{52}$, K.Muenich ${ }^{52}$, M.Mulders ${ }^{30}$, C.Mulet-Marquis ${ }^{14}$, R.Muresan $^{24}$, W.J.Murray ${ }^{36}$, B.Muryn ${ }^{14,18}$, G.Myatt ${ }^{34}$, T.Myklebust ${ }^{32}$. F.Naraghi ${ }^{14}$. F.L.Navarria ${ }^{5}$. S.Navas ${ }^{49}$ ', K.Nawrocki ${ }^{51}$, P.Negri ${ }^{27}$, N.Neufeld ${ }^{9}$, N.Neumeister ${ }^{50}$, R.Nicolaidou ${ }^{39}$, B.S.Nielsen ${ }^{28}$, M.Nikolenko ${ }^{10,16}$, V.Nomokonov ${ }^{15}$, A.Normand ${ }^{22}$, A.Nygren ${ }^{24}$, V.Obraztsov ${ }^{42}$, A.G.Olshevski ${ }^{16}$, A.Onofre ${ }^{21}$, R.Orava ${ }^{15}$, G.Orazi $^{10}$, K.Osterberg ${ }^{15}$, A.Ouraou ${ }^{39}$, M.Paganoni ${ }^{27}$, S.Paiano ${ }^{5}$, R.Pain ${ }^{23}$, R.Paiva ${ }^{21}$, J.Palacios ${ }^{34}$, H.Palka ${ }^{18}$, Th.D.Papadopoulou ${ }^{31}$, K.Papageorgiou ${ }^{11}$, L.Pape ${ }^{9}$, C.Parkes ${ }^{9}$, F.Parodi ${ }^{13}$, U.Parzefall ${ }^{22}$, A.Passeri $^{38}$,

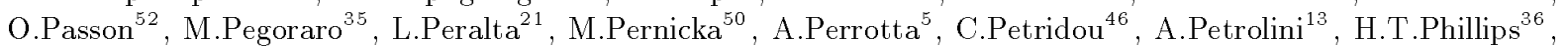
F.Pierre $^{39}$, M.Pimenta ${ }^{21}$, E.Piotto ${ }^{27}$, T.Podobnik ${ }^{43}$, M.E.Pol ${ }^{6}$, G.Polok ${ }^{18}$, P.Poropat ${ }^{46}$, V.Pozdniakov ${ }^{16}$, P.Privitera $^{37}$, N.Pukhaeva ${ }^{16}$, A.Pullia ${ }^{27}$, D.Radojicic ${ }^{34}$, S.Ragazzi $^{27}$, H.Rahmani $^{31}$, D.Rakoczy ${ }^{50}$, P.N.Ratoff ${ }^{20}$, A.L.Read ${ }^{32}$, P.Rebecchi ${ }^{9}$, N.G.Redaelli ${ }^{27}$, M.Regler $^{50}$, D.Reid ${ }^{30}$, R.Reinhardt ${ }^{52}$, P.B.Renton ${ }^{34}$, L.K.Resvanis ${ }^{3}$, F.Richard ${ }^{19}$, J.Ridky ${ }^{12}$, G.Rinaudo ${ }^{45}$, O.Rohne ${ }^{32}$, A.Romero ${ }^{45}$, P.Ronchese ${ }^{35}$, E.I.Rosenberg ${ }^{1}$, P.Rosinsky ${ }^{7}$, P.Roudeau $^{19}$, T.Rovelli ${ }^{5}$, Ch.Royon ${ }^{39}$, V.Ruhlmann-Kleider ${ }^{39}$, A.Ruiz ${ }^{40}$, H.Saarikko ${ }^{15}$, Y.Sacquin ${ }^{39}$, A.Sadovsky ${ }^{16}$, G.Sajot $^{14}$, J.Salt ${ }^{49}$, D.Sampsonidis ${ }^{11}$, M.Sannino ${ }^{13}$, H.Schneider ${ }^{17}$, Ph.Schwemling $^{23}$, U.Schwickerath $^{17}$, M.A.E.Schyns ${ }^{52}$,F.Scuri ${ }^{46}$, P.Seager $^{20}$, Y.Sedykh ${ }^{16}$, A.M.Segar ${ }^{34}$, R.Sekulin ${ }^{36}$, V.Senko $^{42}$, R.C.Shellard ${ }^{6}$, A.Sheridan ${ }^{22}$, M.Siebel $^{52}$, L.Simard ${ }^{39}$, F.Simonetto ${ }^{35}$, A.N.Sisakian ${ }^{16}$, G.Smadja $^{25}$, N.Smirnov ${ }^{42}$, O.Smirnova ${ }^{24}$, G.R.Smith ${ }^{36}$, O.Solovianov ${ }^{42}$, A.Sopczak ${ }^{17}$, R.Sosnowski ${ }^{51}$, T.Spassov ${ }^{21}$, E.Spiriti $^{38}$. P.Sponholz ${ }^{52}$, S.Squarcia ${ }^{13}$. D.Stampfer ${ }^{50}$, C.Stanescu ${ }^{38}$. S.Stanic ${ }^{43}$, K.Stevenson ${ }^{34}$. A.Stocchi ${ }^{19}$, J.Strauss $^{50}$, R.Strub ${ }^{10}$, B.Stugu ${ }^{4}$, M.Szczekowski ${ }^{51}$, M.Szeptycka ${ }^{51}$, T.Tabarelli ${ }^{27}$, O.Tchikilev ${ }^{42}$, F.Tegenfeldt ${ }^{48}$, F.Terranova ${ }^{27}$, J.Thomas ${ }^{34}$, J.Timmermans ${ }^{30}$, N.Tinti ${ }^{5}$, L.G.Tkatchev ${ }^{16}$, S.Todorova ${ }^{10}$, A.Tomaradze $^{2}$, B.Tome ${ }^{21}$, A.Tonazzo ${ }^{9}$, L.Tortora $^{38}$, G.Transtromer ${ }^{24}$, D.Treille ${ }^{9}$, G.Tristram ${ }^{8}$, 
M.Trochimczuk ${ }^{51}$, C.Troncon ${ }^{27}$, A.Tsirou ${ }^{9}$, M-L.Turluer ${ }^{39}$, I.A.Tyapkin ${ }^{16}$, S.Tzamarias ${ }^{11}$, B.Ueberschaer ${ }^{52}$, O.Ullaland $^{9}$, V.Uvarov ${ }^{42}$, G.Valenti ${ }^{5}$, E.Vallazza ${ }^{46}$, C.Vander Velde ${ }^{2}$, G.W.Van Apeldoorn ${ }^{30}$, P.Van Dam ${ }^{30}$, W.K.Van Doninck ${ }^{2}$, J.Van Eldik ${ }^{30}$, A.Van Lysebetten ${ }^{2}$, I.Van Vulpen ${ }^{30}$, N.Vassilopoulos ${ }^{34}$, G.Vegni ${ }^{27}$, L.Ventura $^{35}$, W.Venus ${ }^{36,9}$, F.Verbeure ${ }^{2}$, M.Verlato ${ }^{35}$, L.S.Vertogradov ${ }^{16}$, V.Verzi ${ }^{37}$, D.Vilanova ${ }^{39}$, N.Vishnevsky ${ }^{42}$, L.Vitale ${ }^{46}$, E.Vlasov ${ }^{42}$, A.S.Vodopyanov ${ }^{16}$, C.Vollmer ${ }^{17}$, G.Voulgaris ${ }^{3}$, V.Vrba ${ }^{12}$, H.Wahlen ${ }^{52}$, C.Walck $^{44}$, C.Weiser ${ }^{17}$, D.Wicke ${ }^{52}$, J.H.Wickens ${ }^{2}$, G.R.Wilkinson ${ }^{9}$, M.Winter $^{10}$, M.Witek ${ }^{18}$, G.Wolf ${ }^{9}, J$.Yi ${ }^{1}$, A.Zalewska $^{18}$, P.Zalewski ${ }^{51}$, D.Zavrtanik ${ }^{43}$, E.Zevgolatakos ${ }^{11}$, N.I.Zimin ${ }^{16,24}$, G.C.Zucchelli4 ${ }^{44}$, G.Zumerle ${ }^{35}$

${ }^{1}$ Department of Physics and Astronomy, Towa State University, Ames IA 50011-3160, USA

${ }^{2}$ Physics Department, Univ. Instelling Antwerpen, Universiteitsplein 1, BE-2610 Wilrijk, Belgium and IIHE, ULB-VUB, Pleinlaan 2, BE-1050 Brussels, Belgium

and Faculté des Sciences, Univ. de l'Etat Mons, Av. Maistriau 19, BE-7000 Mons, Belgium

${ }^{3}$ Physics Laboratory, University of Athens, Solonos Str. 104, GR-10680 Athens, Greece

${ }^{4}$ Department of Physics, University of Bergen, Allégaten 55, NO-5007 Bergen, Norway

${ }^{5}$ Dipartimento di Fisica, Università di Bologna and INFN, Via Irnerio 46, IT-40126 Bologna, Italy

${ }^{6}$ Centro Brasileiro de Pesquisas Físicas, rua Xavier Sigaud 150, BR-22290 Rio de Janeiro, Brazil

and Depto. de Física, Pont. Univ. Católica, C.P. 38071 BR-22453 Rio de Janeiro, Brazil

and Inst. de Física, Univ. Estadual do Rio de Janeiro, rua São Francisco Xavier 524, Rio de Janeiro, Brazil

${ }^{7}$ Comenius University, Faculty of Mathematics and Physics, Mlynska Dolina, SK-84215 Bratislava, Slovakia

${ }^{8}$ Collège de France, Lab. de Physique Corpusculaire, IN2P3-CNRS, FR-75231 Paris Cedex 05, France

${ }^{9} \mathrm{CERN}, \mathrm{CH}-1211$ Geneva 23, Switzerland

${ }^{10}$ Institut de Recherches Subatomiques, IN2P3 - CNRS/ULP - BP20, FR-67037 Strasbourg Cedex, France

${ }^{11}$ Institute of Nuclear Physics, N.C.S.R. Demokritos, P.O. Box 60228, GR-15310 Athens, Greece

${ }^{12}$ FZU, Inst. of Phys. of the C.A.S. High Energy Physics Division, Na Slovance 2, CZ-180 40, Praha 8 , Czech Republic

${ }^{13}$ Dipartimento di Fisica, Università di Genova and INFN, Via Dodecaneso 33, IT-16146 Genova, Italy

${ }^{14}$ Institut des Sciences Nucléaires, IN2P3-CNRS, Université de Grenoble 1, FR-38026 Grenoble Cedex, France

${ }^{15}$ Helsinki Institute of Physics, HIP, P.O. Box 9, FI-00014 Helsinki, Finland

${ }^{16}$ Joint Institute for Nuclear Research, Dubna, Head Post Office, P.O. Box 79, RU-101 000 Moscow, Russian Federation

${ }^{17}$ Institut für Experimentelle Kernphysik, Universität Karlsruhe, Postfach 6980, DE-76128 Karlsruhe, Germany

${ }^{18}$ Institute of Nuclear Physics and University of Mining and Metalurgy, Ul. Kawiory 26a, PL-30055 Krakow, Poland

${ }^{19}$ Université de Paris-Sud, Lab. de l'Accélérateur Linéaire, IN2P3-CNRS, Bât. 200, FR-91405 Orsay Cedex, France

${ }^{20}$ School of Physics and Chemistry, University of Lancaster, Lancaster LA1 4YB, UK

${ }^{21}$ LIP, IST, FCUL - Av. Elias Garcia, 14-1 ${ }^{\circ}$, PT-1000 Lisboa Codex, Portugal

${ }^{2}$ Department of Physics, University of Liverpool, P.O. Box 147, Liverpool L69 3BX, UK

${ }^{23}$ LPNHE, IN2P3-CNRS, Univ. Paris VI et VII, Tour 33 (RdC), 4 place Jussieu, FR-75252 Paris Cedex 05, France

${ }^{24}$ Department of Physics, University of Lund, Sölvegatan 14, SE-223 63 Lund, Sweden

${ }^{25}$ Université Claude Bernard de Lyon, IPNL, IN2P3-CNRS, FR-69622 Villeurbanne Cedex, France

${ }^{26}$ Univ. d'Aix - Marseille II - CPP, IN2P3-CNRS, FR-13288 Marseille Cedex 09, France

${ }^{27}$ Dipartimento di Fisica, Università di Milano and INFN, Via Celoria 16, IT-20133 Milan, Italy

${ }^{28}$ Niels Bohr Institute, Blegdamsvej 17, DK-2100 Copenhagen $\emptyset$, Denmark

${ }^{29} \mathrm{NC}$, Nuclear Centre of MFF, Charles University, Areal MFF, V Holesovickach 2, CZ-180 00, Praha 8, Czech Republic

${ }^{30}$ NIKHEF, Postbus 41882, NL-1009 DB Amsterdam, The Netherlands

${ }^{31}$ National Technical University, Physics Department, Zografou Campus, GR-15773 Athens, Greece

${ }^{32}$ Physics Department, University of Oslo, Blindern, NO-1000 Oslo 3, Norway

${ }^{33}$ Dpto. Fisica, Univ. Oviedo, Avda. Calvo Sotelo s/n, ES-33007 Oviedo, Spain

${ }^{34}$ Department of Physics, University of Oxford, Keble Road, Oxford OX1 3RH, UK

${ }^{35}$ Dipartimento di Fisica, Università di Padova and INFN, Via Marzolo 8, IT-35131 Padua, Italy

${ }^{36}$ Rutherford Appleton Laboratory, Chilton, Didcot OX11 OQX, UK

${ }^{37}$ Dipartimento di Fisica, Università di Roma II and INFN, Tor Vergata, IT-00173 Rome, Italy

${ }^{38}$ Dipartimento di Fisica, Università di Roma III and INFN, Via della Vasca Navale 84, IT-00146 Rome, Italy

39 DAPNIA/Service de Physique des Particules, CEA-Saclay, FR-91191 Gif-sur-Yvette Cedex, France

${ }^{40}$ Instituto de Fisica de Cantabria (CSIC-UC), Avda. los Castros s/n, ES-39006 Santander, Spain

${ }^{41}$ Dipartimento di Fisica, Università degli Studi di Roma La Sapienza, Piazzale Aldo Moro 2, IT-00185 Rome, Italy

${ }^{42}$ Inst. for High Energy Physics, Serpukov P.O. Box 35, Protvino, (Moscow Region), Russian Federation

${ }^{43}$ J. Stefan Institute, Jamova 39, SI-1000 Ljubljana, Slovenia and Laboratory for Astroparticle Physics,

Nova Gorica Polytechnic, Kostanjeviska 16a, SI-5000 Nova Gorica, Slovenia,

and Department of Physics, University of Ljubljana, SI-1000 Ljubljana, Slovenia

${ }^{44}$ Fysikum, Stockholm University, Box 6730, SE-113 85 Stockholm, Sweden

${ }^{45}$ Dipartimento di Fisica Sperimentale, Università di Torino and INFN, Via P. Giuria 1, IT-10125 Turin, Italy

${ }^{46}$ Dipartimento di Fisica, Università di Trieste and INFN, Via A. Valerio 2, IT-34127 Trieste, Italy and Istituto di Fisica, Università di Udine, IT-33100 Udine, Italy

${ }^{47}$ Univ. Federal do Rio de Janeiro, C.P. 68528 Cidade Univ., Ilha do Fundão BR-21945-970 Rio de Janeiro, Brazil

${ }^{48}$ Department of Radiation Sciences, University of Uppsala, P.O. Box 535, SE-751 21 Uppsala, Sweden

${ }^{49}$ IFIC, Valencia-CSIC, and D.F.A.M.N., U. de Valencia, Avda. Dr. Moliner 50, ES-46100 Burjassot (Valencia), Spain

${ }^{50}$ Institut für Hochenergiephysik, Österr. Akad. d. Wissensch., Nikolsdorfergasse 18, AT-1050 Vienna, Austria

${ }^{51}$ Inst. Nuclear Studies and University of Warsaw, Ul. Hoza 69, PL-00681 Warsaw, Poland

${ }^{52}$ Fachbereich Physik, University of Wuppertal, Postfach 100 127, DE-42097 Wuppertal, Germany

${ }_{53}$ On leave of absence from IHEP Serpukhov

${ }^{54}$ Now at University of Florida 


\section{Introduction}

At the end of 1995 and in 1996 LEP was run for the first time at energies well above the $Z^{0}$ resonance, the LEP-2 regime. A total luminosity of about $26 \mathrm{pb}^{-1}$ was collected at centre-of-mass energies ranging from 130 to $172 \mathrm{GeV}$, allowing the determination of the cross-sections for the inclusive production of quark-antiquark pairs and for lepton pairs of each flavour in a new kinematic domain. Lepton pairs were also used to extract forwardbackward charge asymmetries. In addition, the cross-section ratios and forward-backward asymmetries for light (uds), $c$ and b quarks have been determined, using flavour-tagging techniques.

A characteristic feature of fermion pair production at these energies is that a large part of the events undergo Initial State Radiation (ISR), which reduces the effective centre-of-mass energy, $\sqrt{s^{\prime}}$; in particular down to $Z^{0}$ energies.

The determination of the luminosity and the selection of the different final states were similar to those used at LEP-1 [1,2]. However, the selection criteria were adapted to the fast decrease of the signal cross-sections with increasing collision energy and to the emergence of new backgrounds due to four-fermion production. For each collision energy, the hadronic and leptonic cross-sections and the leptonic forward-backward asymmetries were computed for the full range of $\sqrt{s^{\prime}}$, as well as for the subsample of events where $\sqrt{s^{\prime}}$ was close to the collision energy $(\sqrt{s})$.

A description of the DELPHI apparatus is given in [3] and details on the performance of the DELPHI detector and algorithms used for reconstruction and simulation can be found in [4]. The specific event generators used for the analyses are described in the relevant sections of this paper. The details of the LEP energy measurement, luminosity determination, the computation of $s^{\prime}$ and the analyses of each final state, i.e. inclusive $e^{+} e^{-}, \mu^{+} \mu^{-}, \tau^{+} \tau^{-}$and $q \bar{q}$ pairs, are given in section 2 . In addition to the inclusive quark-antiquark cross-section data, measurements of the cross-sections for light (u,d,s), charm (c) and bottom (b) quarks, and of their forward-backward asymmetries, have been made. The analysis techniques used in extracting these flavour-tagged samples are also described in section 2 .

The results on the lepton data and inclusive $q \bar{q}$ are given in section 3 , together with results on the cross-sections and asymmetries of flavour-tagged hadronic final states. The cross-sections and asymmetries measured at high energies were combined with the published $Z^{0}$ data $[1,2]$ in order to check the predictions of the Standard Model (SM). The data were analysed in the framework of the S-matrix approach, achieving a substantial improvement in the precision of the hadronic $\gamma Z^{0}$ interference compared to the accuracy obtained from the $Z^{0}$ data alone, this is discussed in section 4 .

The data are also interpreted in terms of several models which include physics beyond the SM, the theoretical descriptions of these models are given in section 5 and the results of the interpretations are given in section 6. Many of these models predict sizeable effects in $e^{+} e^{-}$collisions at energies above the $\mathrm{Z}$ resonance. For example, several models proposed to explain the anomaly reported by the HERA experiments [5] also predict deviations from the SM for observables at LEP-2 (e.g. [6,7]). The first set of models considered here parameterise new physics, with a characteristic high energy scale, in terms of effective contact interactions between fermions. Non-conservation of R-parity in Supersymmetric extensions of the Standard Model could lead to effects in both the cross-sections and forward-backward asymmetries of $e^{+} e^{-} \rightarrow f \bar{f}$. The high-energy results presented here are used to determine limits on many of the possible R-parity violating couplings. 
Finally, in many extensions to the SM there are additional $\mathrm{Z}^{\prime}$ bosons. Such bosons would give rise to deviations from the SM predictions, both for the cross-sections and forward-backward asymmetries, largely through interference effects with the SM amplitudes. The theoretical framework of these models is discussed in section 5 .

The results of the interpretation of the data presented in this paper in terms of contact interactions and R-parity violating SUSY effects are described in section 6 . This interpretation is performed separately for the lepton cross-section and forward-backward asymmetries and for the flavour-tagged $q \bar{q}$ cross-section and asymmetry data. The high energy data, together with data taken at the Z-pole, are also interpreted in terms of possible Z' bosons.

A summary and conclusions are given in section 7 . For comparison, the results on the analyses of the high energy $e^{+} e^{-} \rightarrow f \bar{f}$ data from the other LEP experiments can be found in [8], [9] and [10].

\section{Measurements of cross-sections and asymmetries}

\subsection{LEP energy determination}

At energies well above the $Z^{0}$ resonance the LEP energy cannot be determined directly by resonant depolarisation. In 1996, resonant depolarisation was achieved at a beam energy of $50 \mathrm{GeV}$. The centre-of-mass energies for the data between 130 and $172 \mathrm{GeV}$ are determined by using a model of the LEP energy $[11,12]$. Information on the magnetic fields of the dipole magnets, the temperature of the LEP dipole magnets, the RF cavities and other quantities which influence the LEP energy are used in this model. The model is normalised to the resonant depolarisation data. For higher energies, an extrapolation must be performed and the uncertainty on the normalisation used in the extrapolation is the largest source of uncertainty. The estimated uncertainties on the LEP beam energies are 27 and $30 \mathrm{MeV}$ at beam energies of 80.5 and $86 \mathrm{GeV}$ respectively [11]. For the data taken in 1995, at beam energies between 65 and $70 \mathrm{GeV}$, the estimated uncertainty on the beam energy is $25 \mathrm{MeV}[12]$.

\subsection{Luminosity measurement}

The luminosity was derived from the rate of events due to Bhabha scattering reconstructed in the high precision Small angle TIle Calorimeter (STIC) of the experiment, which consists of two lead scintillator sampling calorimeters. Located at $\pm 220 \mathrm{~cm}$ from the interaction point, they provide full coverage of the region between 29 and $185 \mathrm{mrad}$ with respect to the beam line. A detailed description of the detector can be found in [13].

The events due to Bhabha scattering were selected by demanding a coincidence of two showers, coplanar with the beam direction and with energies larger than $65 \%$ of the beam energy, and by requiring that the reconstructed radial position of the showers were inside the geometrical acceptance.

Due to a very sharp angular dependence of the Bhabha cross-section at small angles the uncertainty on the inner edge of the acceptance represents the major experimental uncertainty in luminosity determination. In 1995 the inner radius of the acceptance on one side was defined by a precisely machined conical tungsten mask projecting to the interaction point, which absorbs incoming electrons. At the start of 1996, the mask was removed to increase the acceptance for four-fermion processes. The selection of Bhabha 
events was therefore based on the radius of the showers reconstructed in both calorimeters. In order to reduce the dependence of the visible cross-section on the longitudinal position of the interaction point the side with tighter cuts on radial position of showers was alternated at each trigger. The loss in precision after the removal of the mask resulted in an increase of the total experimental systematic uncertainty on the luminosity determination from $0.09 \%$ in 1995 to $0.5 \%$ in 1996 . This value is still small when compared to the statistical precision of the measured cross-sections for fermion pair production.

The calculation of the visible cross-section was based on the event generator BHLUMI 4.03 [14], which has a theoretical accuracy of $\pm 0.25 \%$.

Experimental and theoretical systematic uncertainties on the luminosity are not included in the errors on the individual cross-section measurements presented hereafter. They are treated as common to all cross-section measurements in the fitting procedure.

\subsection{Determination of $\mathrm{s}^{\prime}$}

Although slightly different for each final state, the computations of $\sqrt{s^{\prime}}$ were all performed using the constraint of the collision energy, the reconstructed directions of the final state fermions and the information of the electromagnetic calorimeters on isolated (ISR) energetic photons. The performance of all $\sqrt{s^{\prime}}$ computation methods and the systematic errors associated to the separation of the non-radiative part of measurements were estimated from simulated events.

In the $e^{+} e^{-} \rightarrow q \bar{q}(\gamma)$ analysis, each event was first forced into a 2-jet configuration by adjusting the value of the parameter $d_{j o i n}$ in the LUCLUS [15] clusterisation algorithm. The value of $\sqrt{s^{\prime}}$ was derived from the polar angles of the jet directions $\left(\theta_{1}, \theta_{2}\right)$, assuming that a single ISR photon was emitted along the beam line. In this topology the reduced centre-of-mass energy is given by the following expression:

$$
s^{\prime}=s-2 E_{\gamma} \sqrt{s},
$$

where $E_{\gamma}$ is the ISR photon energy:

$$
E_{\gamma}=\frac{\left|\sin \left(\theta_{1}+\theta_{2}\right)\right| \sqrt{s}}{\sin \theta_{1}+\sin \theta_{2}+\left|\sin \left(\theta_{1}+\theta_{2}\right)\right|} .
$$

When an isolated energetic photon was reconstructed in the electromagnetic calorimeters the value of $\sqrt{s^{\prime}}$ was computed from the measured photon energy. The fraction of events where such an isolated photon was observed was close to $25 \%$, in agreement with the prediction of a Monte Carlo simulation.

In the $e^{+} e^{-} \rightarrow \mu^{+} \mu^{-}(\gamma)$ analysis, $\sqrt{s^{\prime}}$ was calculated from a kinematic fit procedure, where four different topologies were investigated for each event: i) no photon radiated, ii) one photon radiated along the beam line, iii) one seen and one unseen photon in any direction, iv) a single unseen photon in any direction. The seen photon fit was performed if a neutral energy deposit greater than $5 \mathrm{GeV}$ was measured in the electromagnetic calorimeters. A probability was assigned to each of the four hypotheses on the basis of $\chi^{2}$ of the kinematic fit. The most probable hypothesis was retained, and $\sqrt{s^{\prime}}$ was set accordingly, either to the fitted invariant mass of the muons (topology ii), iii) or iv)), or to $\sqrt{s}($ topology i)).

In the $e^{+} e^{-} \rightarrow \tau^{+} \tau^{-}(\gamma)$ analysis, $\sqrt{s^{\prime}}$ was calculated from the estimated fermion directions using formulae 1 and 2. For the $e^{+} e^{-} \rightarrow e^{+} e^{-}(\gamma)$ channel the analysis is performed in terms of the acollinearity of the outgoing electron and positron, which is well determined experimentally and can be treated theoretically. The acollinearity is 
correlated to $\mathrm{s}^{\prime}$ in $s$-channel processes, so that a cut on acollinearity can be used to select events with predominantly low energy initial state radiation.

\section{$2.4 e^{+} e^{-} \rightarrow e^{+} e^{-}(\gamma)$ in the central angular region}

The analysis was similar to that used for $Z^{0}$ energies with the values of the cuts imposed on energy and momenta scaled according to the centre-of-mass energy. The details of the event selection can be found in [1,2].

The electron and positron were required to be in the polar angle range $44^{\circ}<\theta<136^{\circ}$ and the non-radiative events were selected by requiring the acollinearity angle between the final state $\mathrm{e}^{+}$and $\mathrm{e}^{-}, \theta_{\text {acol }}$, to be smaller than $20^{\circ}{ }^{1}$. In this region the statistics were sufficient to apply the method where the selection efficiency is derived from the data themselves, using two independent selections based on the information delivered by different sets of subdetectors (one using the barrel electromagnetic calorimeter and the silicon vertex detector, and the other using the main tracking detectors in the barrel region, namely, the Inner Detector, the Time Projection Chamber and the Outer Detector).

Another analysis which estimates efficiency from simulated events was performed for the acollinearity region between $20^{\circ}$ and $90^{\circ}$. It was checked that this method also gives consistent results for the region of acollinearity below $20^{\circ}$. The sum of the results of both methods was used to produce the cross-section and asymmetry for an acollinearity smaller than $90^{\circ}$. This cut defines the total cross-section and asymmetry measurements for the $e^{+} e^{-} \rightarrow e^{+} e^{-}(\gamma)$ process in analogy with the low s' cut for other channels.

The main background was due to $e^{+} e^{-} \rightarrow \tau^{+} \tau^{-}(\gamma)$ events. Its contribution was estimated from simulation. The other sources of background, as well as the feed-through from radiative events into the non-radiative sample, were found to be negligible.

Systematic errors on the cross-section measurements arise from event selection, background subtraction and acceptance definition. The efficiencies, residual backgrounds, contamination of radiative events in the non-radiative sample and total systematic errors of the cross-section analyses are given in Table 1 for each collision energy.

The forward-backward asymmetries have been determined with the same samples of events. Systematic errors on the asymmetry arise from charge confusion and forwardbackward acceptance differences. They are negligible compared to the statistical precision.

\subsection{Forward $e^{+} e^{-} \rightarrow e^{+} e^{-}(\gamma)$}

The differential $e^{+} e^{-}$cross-section was measured in the forward region using the Forward Electromagnetic Calorimeter (FEMC). Events were selected by requiring at least one electromagnetic cluster with an energy greater than $2 / 3$ of the beam energy in each of the calorimeters (polar angle range $12^{\circ}<\theta<35^{\circ}$ and $145^{\circ}<\theta<168^{\circ}$ ) and an acollinearity between the two clusters smaller than 10 degrees.

The selection efficiency was derived from the real data by studying events where only one lepton passed the energy requirements. Simulated events were used to take into account correlations between hemispheres.

The most relevant background was due to $e^{+} e^{-} \rightarrow \gamma \gamma(\gamma)$ events, and amounted to $(1.4 \pm 0.1) \%$. The efficiencies, backgrounds and total systematic errors at each energy point are summarised in table 2.

\footnotetext{
${ }^{1}$ The cut at $20^{\circ}$ corresponds approximately to selecting events where the invariant mass of the ee pair is $\sqrt{s^{\prime}}>0.85 \sqrt{s}$.
} 


\begin{tabular}{|l|c|c|c|c|}
\hline \multicolumn{4}{|c|}{$e^{+} e^{-} \rightarrow e^{+} e^{-}(\gamma), \boldsymbol{\theta}_{\text {acol }}<\mathbf{2 0}^{\circ}$} \\
\hline \multicolumn{1}{|c|}{ Energy $(\mathrm{GeV})$} & 130.2 & 136.2 & 161.3 & 172.1 \\
\hline \hline Efficiency, \% & 97.3 & 98.6 & 97.3 & 97.0 \\
Background, \% & 0.16 & 0.15 & 0.14 & 0.10 \\
Total systematic error, \% & \pm 1.9 & \pm 1.5 & \pm 1.5 & \pm 1.2 \\
\hline
\end{tabular}

\begin{tabular}{|l|c|c|c|c|}
\hline \multicolumn{4}{|c|}{$e^{+} e^{-} \rightarrow e^{+} e^{-}(\gamma), \theta_{\text {acol }}<90^{\circ}$} \\
\hline \multicolumn{1}{|c|}{ Energy $(\mathrm{GeV})$} & 130.2 & 136.2 & 161.3 & 172.1 \\
\hline \hline Efficiency, \% & 96.4 & 97.5 & 95.6 & 95.6 \\
Background, \% & 0.5 & 0.4 & 0.2 & 0.3 \\
Total systematic error, \% & \pm 1.7 & \pm 1.4 & \pm 1.4 & \pm 1.1 \\
\hline
\end{tabular}

Table 1: Efficiencies, residual backgrounds and total systematic errors of the cross-section measurements for $e^{+} e^{-} \rightarrow e^{+} e^{-}(\gamma)$ channel in the central angular region for different collision energies.

\begin{tabular}{|l|c|c|c|c|}
\hline \multicolumn{4}{|c|}{$e^{+} e^{-} \rightarrow e^{+} e^{-}(\gamma), \theta_{\text {acol }}<\mathbf{1 0}^{\circ}$} \\
\hline \multicolumn{1}{|c|}{ Energy $(\mathrm{GeV})$} & 130.2 & 136.2 & 161.3 & 172.1 \\
\hline \hline Efficiency, \% & 83.0 & 82.9 & 82.8 & 82.8 \\
Background, \% & 1.4 & 1.4 & 1.4 & 1.4 \\
Total systematic error, \% & \pm 2.6 & \pm 2.6 & \pm 2.8 & \pm 2.8 \\
\hline
\end{tabular}

Table 2: Efficiencies, residual backgrounds and total systematic errors of the cross-section measurements for $e^{+} e^{-} \rightarrow e^{+} e^{-}(\gamma)$ channel in the forward region for different collision energies. 


\begin{tabular}{|l|c|c|c|c|}
\hline \multicolumn{5}{|c|}{$e^{+} e^{-} \rightarrow \boldsymbol{\mu}^{+} \boldsymbol{\mu}^{-}(\gamma), \sqrt{\mathrm{s}^{\prime}} / \sqrt{\mathrm{s}}>\mathbf{0 . 8 5}$} \\
\hline \multicolumn{1}{|c|}{ Energy $(\mathrm{GeV})$} & 130.2 & 136.2 & 161.3 & 172.1 \\
\hline Efficiency, \% & 93.0 & 93.0 & 91.5 & 92.5 \\
Background, \% & 3.2 & 3.2 & 3.4 & 2.2 \\
Feed-through from rad.events, \% & 1.8 & 1.8 & 1.5 & 0.9 \\
Total systematic error, \% & \pm 3.7 & \pm 3.7 & \pm 3.5 & \pm 3.4 \\
\hline
\end{tabular}

\begin{tabular}{|l|c|c|c|c|}
\hline \multicolumn{5}{|c|}{$\boldsymbol{e}^{+} \boldsymbol{e}^{-} \rightarrow \boldsymbol{\mu}^{+} \boldsymbol{\mu}^{-}(\gamma), \sqrt{\boldsymbol{s}^{\prime}}>\mathbf{7 5} \mathbf{G e V}$} \\
\hline \multicolumn{1}{|c|}{ Energy $(\mathrm{GeV})$} & 130.2 & 136.2 & 161.3 & 172.1 \\
\hline \hline Efficiency, \% & 91.0 & 91.0 & 91.0 & 91.0 \\
Background, \% & 2.5 & 2.4 & 3.9 & 4.5 \\
Total systematic error, \% & \pm 3.4 & \pm 3.4 & \pm 3.4 & \pm 3.4 \\
\hline
\end{tabular}

Table 3: Efficiencies, residual backgrounds, feed-through from radiative events into the non-radiative sample and total systematic errors of the cross-section measurements for $e^{+} e^{-} \rightarrow \mu^{+} \mu^{-}(\gamma)$ channel for different collision energies.

\section{$2.6 e^{+} e^{-} \rightarrow \mu^{+} \mu^{-}(\gamma)$}

The event selection for the process $e^{+} e^{-} \rightarrow \mu^{+} \mu^{-}(\gamma)$ was based on criteria similar to those used at $Z^{0}$ energies. An event was required to have two identified muons in the polar angle range $20^{\circ} \leq \theta \leq 160^{\circ}$, originating from close to the interaction region (to reduce the cosmic ray background), with the momentum of the most energetic muon being at least $30 \mathrm{GeV} / c$. The contamination of the final sample by two-photon collision events, cosmic rays, $\tau^{+} \tau^{-}$and four-fermion final states was further reduced by requiring the $\mu^{+} \mu^{-}$invariant mass to be greater than $75 \mathrm{GeV} / c^{2}$. After these selections a small background contamination was left, with the main source from cosmic ray events. This was estimated by extrapolating the number of events which were not sufficiently close to the interaction region into the region from which signal events were selected. At $161 \mathrm{GeV}$ and $172 \mathrm{GeV}$ there was a significant contribution from two-photon interactions. At $172 \mathrm{GeV}$, above the threshold for $\mathrm{W}$ pair production, there was also a significant contribution from four-fermion final states.

The non-radiative events were selected by requiring $\sqrt{\mathrm{s}^{\prime}} / \sqrt{\mathrm{s}} \geq 0.85$. The contamination from $Z^{0}$ radiative events was found to decrease with increasing $\sqrt{s}$ and was between $1.8 \%$ and $0.9 \%$. The decrease was due to the increasing separation between the high energy and radiative return peaks with $\sqrt{s}$. The distributions of $\sqrt{\mathrm{s}^{\prime}} / \sqrt{\mathrm{s}}$ obtained for the data and simulation are shown in Figure 1.

The selection efficiency was estimated from simulation and was found to be in agreement with the results of another method based on the data themselves. The resulting cross-sections were corrected to the full angular acceptance using correction factors obtained from DYMU3 [17] and ZFITTER [18]. The efficiencies, residual backgrounds, 


\section{DELPHI}
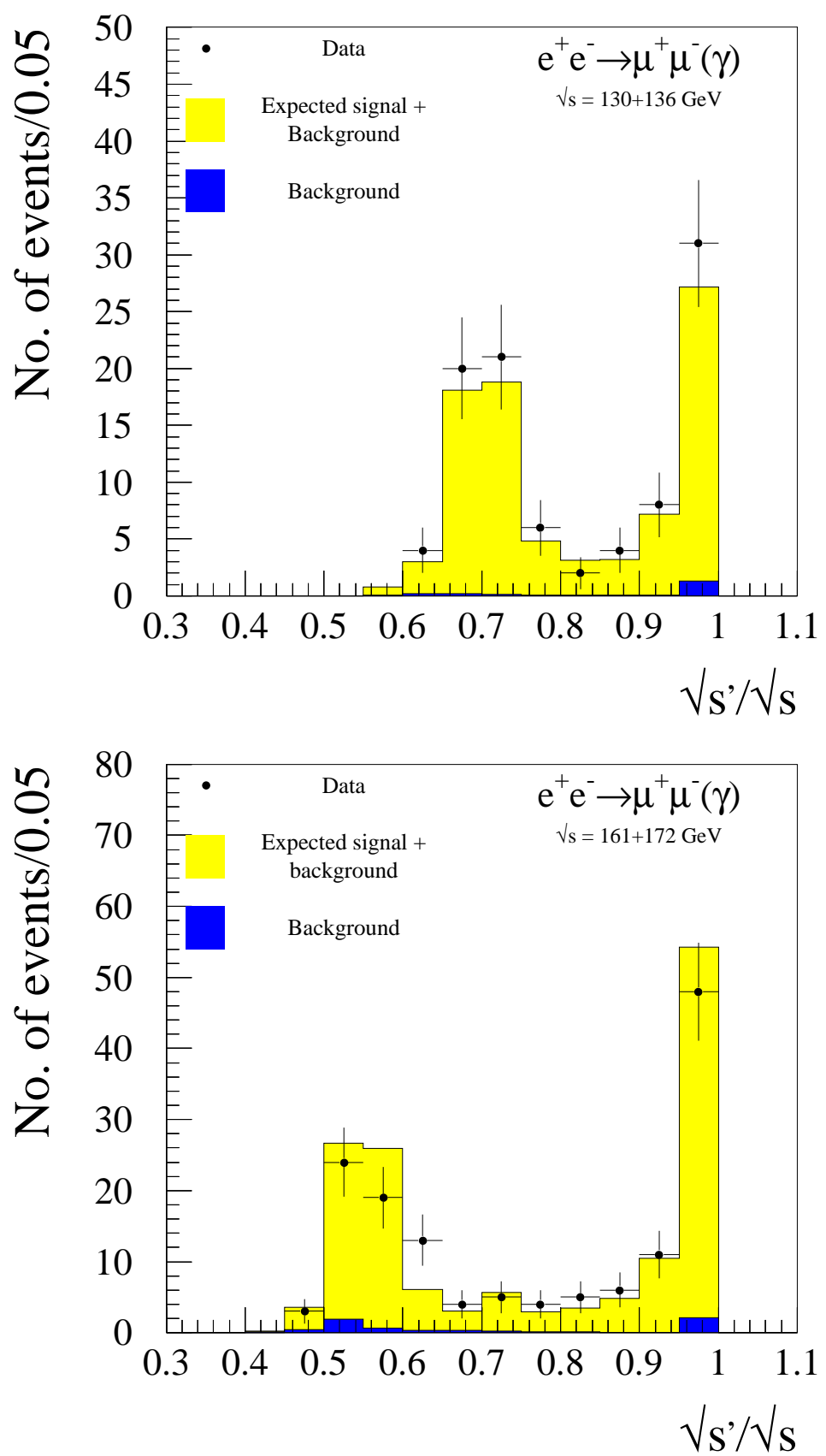

Figure 1: Distribution of the reconstructed reduced energy for the $e^{+} e^{-} \rightarrow \mu^{+} \mu^{-}(\gamma)$ process. The points are the data and the histogram shows the simulation from KORALZ normalised to ZFITTER. 
contamination of radiative events in the non-radiative sample and total systematic errors of the cross-section analyses for different collision energies are given in Table 3.

The forward-backward asymmetry was determined with a counting method: $A_{f b}=$ $\left(N_{f}-N_{b}\right) /\left(N_{f}+N_{b}\right)$, where $N_{f}$ and $N_{b}$ are the number of events with the $\mu^{-}$produced in the forward and the backward region, respectively. Measured asymmetries were corrected for the background and for the contamination of non-radiative events by events with hard ISR photons. The correction to the full angular range was performed using DYMU3.

\section{$2.7 \quad e^{+} e^{-} \rightarrow \tau^{+} \tau^{-}(\gamma)$}

For the selection of tau pair events, the thrust axis was calculated using the charged particle momenta, and the particles in each event were then assigned to the hemispheres formed by the plane perpendicular to the thrust axis and passing through the origin. The leading charged particle in each hemisphere was required to lie in the polar angle range $|\cos \theta|<0.94$, and the observed charged particle multiplicity was required to be unity in one hemisphere and no more than five in the other. The leading charged particles in both hemispheres had to be consistent with originating from the interaction region and at least one of them was required to have momentum greater than $0.025 \times \sqrt{s} / c$. Non-radiative events were selected by requiring $\sqrt{\mathrm{s}^{\prime}} / \sqrt{\mathrm{s}}>0.85$.

For the rejection of Bhabha events, $\mu$-pairs and cosmic ray backgrounds, the acollinearity was required to be greater than $0.5^{\circ}$. In addition, a radial momentum was defined as $P_{\text {rad }}=\sqrt{x_{1}^{2}+x_{2}^{2}}$, where $x_{1(2)}$ is the momentum of the most energetic charged particle in hemisphere 1(2) normalised to that expected for a dimuon event, $P_{1(2)}$, which was calculated from the formula

$$
P_{1(2)}=\sqrt{s} \sin \theta_{2(1)} /\left(\left|\sin \left(\theta_{1}+\theta_{2}\right)\right|+\sin \theta_{1}+\sin \theta_{2}\right) .
$$

The value of $P_{\text {rad }}$ was required to be less than 1.1. Similarly, a radial energy, $E_{\text {rad }}$, was defined using the total electromagnetic calorimetric energy deposited in a cone of halfangle $30^{\circ}$ around the highest momentum charged particle track in each hemisphere, and its value was required to satisfy $E_{\text {rad }}<0.8$. Dimuon events have a peak at $\sqrt{2}$ in radial momentum while Bhabha events have a peak at $\sqrt{2}$ for both radial momentum and radial energy.

To reject the remaining two-photon background, it was required that the total visible energy of the event exceeded $0.15 \sqrt{s}$, and the transverse momentum of the event be greater than $0.04 \sqrt{s} / \mathrm{c}$ for those events consistent with the reactions $e^{+} e^{-} \rightarrow e^{+} e^{-} e^{+} e^{-}$ and $e^{+} e^{-} \rightarrow e^{+} e^{-} \mu^{+} \mu^{-}$, where both channels were tagged using calorimetric signals. Further rejection of both Bhabha and $e^{+} e^{-} \rightarrow e^{+} e^{-} e^{+} e^{-}$events was obtained by rejecting events where both leading charged particles had an associated electromagnetic energy greater than $40 \%$ of the measured particle momentum.

The background from $e^{+} e^{-} \rightarrow W W, Z Z$ events was reduced by demanding that the event acoplanarity ${ }^{1}$, was less than 0.3 radians.

The distribution of $\sqrt{\mathrm{s}^{\prime}} / \sqrt{\mathrm{s}}$ is presented in Figure 2. The selection efficiency and the background estimation were performed using simulated events. The total and nonradiative cross-sections were determined after correcting for the selection efficiency and background, and for feed-through from radiative events in the case of the non-radiative cross-sections.

\footnotetext{
${ }^{1}$ The event acoplanarity is defined as $\left|\phi_{1}-\phi_{2}\right|-\pi$, where $\phi_{1(2)}$ are the azimuthal angles of the leading tracks in hemispheres 1 (2) in the DELPHI coordinate system, in which the z-axis points along the direction of incoming electrons, the $x$-axis points towards the centre of LEP and the $y$-axis points vertically upwards
} 


\section{DELPHI}
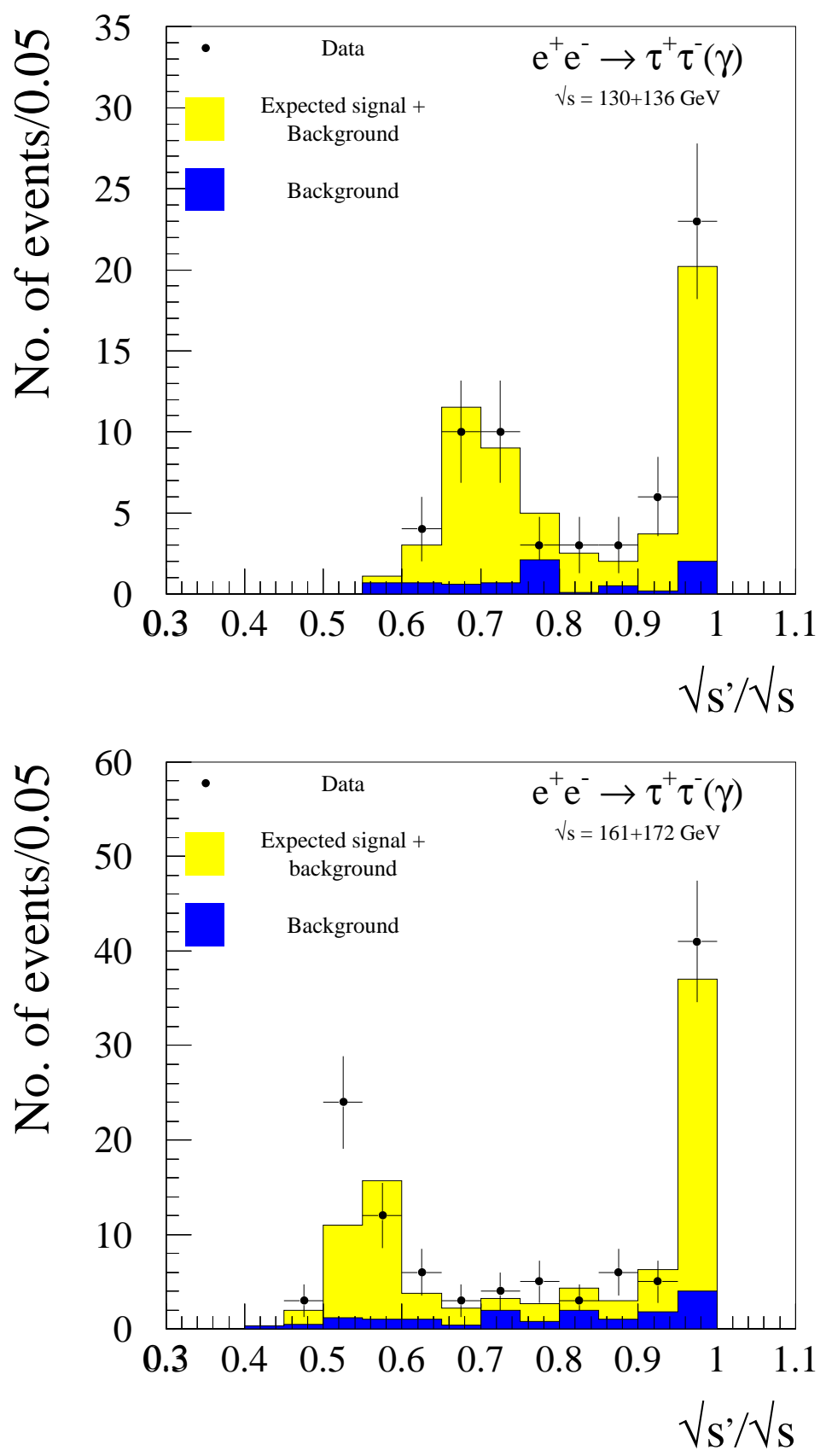

Figure 2: Distribution of the reconstructed reduced energy for the $e^{+} e^{-} \rightarrow \tau^{+} \tau^{-}(\gamma)$ process. The points are the data and the histogram shows the simulation from KORALZ normalised to ZFITTER. 
The efficiencies, residual backgrounds, contamination of radiative events in the nonradiative sample and total systematic errors of the cross-section analyses for different collision energies are given in Table 4.

The forward-backward charge asymmetry was determined using the counting method where the thrust axis defined the polar angle. The asymmetry was corrected for acceptance and background and in the case of the high-energy sample for feed-through from radiative events.

\section{$2.8 \quad$ Inclusive $e^{+} e^{-} \rightarrow q \bar{q}(\gamma)$}

The selection of inclusive hadronic final states was based on the charged particle tracks chosen with the criteria described in $[1,2]$. Events were retained if they contained at least 7 charged particles and if the energy of charged particles was greater than $15 \%$ of the collision energy. In addition, the quantity $\sqrt{E_{F}^{2}+E_{B}^{2}}$, where $E_{F}$ and $E_{B}$ are the total energy seen in the Forward and Backward electromagnetic calorimeters, was required to be less than $90 \%$ of the beam energy.

The selection efficiency was computed from simulated events produced with the PYTHIA 5.7 [15] generator, which was tuned on the data collected by DELPHI around the $Z^{0}[19]$.

The residual background contamination was estimated with simulated event samples. The TWOGAM generator [20] was used to simulate two-photon collisions, PYTHIA and BABAMC [21] were used for lepton pair production, and PYTHIA was used for fourfermion production. Below $161 \mathrm{GeV}$, the main background contributions to the total cross-section measurement came from two-photon interactions and from $Z^{0} e^{+} e^{-}$events (amounting typically to $5.5 \pm 1 \mathrm{pb}$ and $1.8 \pm 0.5 \mathrm{pb}$, respectively). Above $161 \mathrm{GeV}$, W-pair production became a substantial background. It dominates at $172 \mathrm{GeV}$, with a contribution of $10.9 \pm 0.3 \mathrm{pb}$ to the total cross-section and of $4.9 \pm 0.2 \mathrm{pb}$ to the non-radiative cross-section.

The distribution of the reconstructed reduced energy is presented in Figure 3 for the four collision energies. The total cross-section refers to $\sqrt{\mathrm{s}^{\prime}} / \sqrt{\mathrm{s}}>0.1$, whereas the non-radiative cross-section refers to $\sqrt{\mathrm{s}^{\prime}} / \sqrt{\mathrm{s}}>0.85$. The resolution on the latter cut value translates into a purity of the non-radiative event sample which increases from about $82 \%$ at $130 \mathrm{GeV}$ to about $90 \%$ at $172 \mathrm{GeV}$. The systematic uncertainty on the selection efficiency for non-radiative events, which amounts to $\pm 2.5 \%$, was dominated by the accuracy of the determination of $\sqrt{\mathrm{s}^{\prime}} / \sqrt{\mathrm{s}}$ which also includes the uncertainty in the ISR.

The efficiencies, residual backgrounds and contamination of radiative events in the non-radiative sample entering the computation of the cross-sections are given in Table 5 for each collision energy, together with the total systematic uncertainties associated to each measurement.

\subsection{Flavour-tagged hadronic final states}

The hadronic data were analysed to investigate the separate production of bottom, charm and light quarks.

The selection of hadronic events was as described for inclusive hadronic final states. In addition, the thrust axis of the event was calculated including neutral particles and its polar angle was required to be between $25^{\circ}$ and $155^{\circ}$. The charged and neutral particles were clustered into jets using the LUCLUSalgorithm with an invariant mass 


\begin{tabular}{|l|c|c|c|c|}
\hline \multicolumn{5}{|c|}{$\boldsymbol{e}^{+} \boldsymbol{e}^{-} \rightarrow \boldsymbol{\tau}^{+} \boldsymbol{\tau}^{-}(\gamma), \sqrt{\mathbf{s}^{\prime}} / \sqrt{\mathrm{s}}>\mathbf{0 . 8 5}$} \\
\hline \multicolumn{1}{|c|}{ Energy $(\mathrm{GeV})$} & 130.2 & 136.2 & 161.3 & 172.1 \\
\hline \hline Efficiency, \% & 45.8 & 48.7 & 44.2 & 46.0 \\
Background, \% & 15 & 15 & 16 & 12 \\
Feed-through from rad.events, \% & 7 & 6 & 4 & 8 \\
Total systematic error, \% & \pm 7 & \pm 7 & \pm 7 & \pm 7 \\
\hline
\end{tabular}

\begin{tabular}{|l|c|c|c|c|}
\hline \multicolumn{5}{|c|}{$\boldsymbol{e}^{+} \boldsymbol{e}^{-} \rightarrow \boldsymbol{\tau}^{+} \boldsymbol{\tau}^{-}(\boldsymbol{\gamma}), \sqrt{\boldsymbol{s}^{\prime}}>\mathbf{7 5} \mathbf{~ G e V}$} \\
\hline Energy $(\mathrm{GeV})$ & 130.2 & 136.2 & 161.3 & 172.1 \\
\hline \hline Efficiency, \% & 44.1 & 45.8 & 41.6 & 40.8 \\
Background, \% & 17 & 16 & 17 & 16 \\
Total systematic error, \% & \pm 7 & \pm 7 & \pm 7 & \pm 7 \\
\hline
\end{tabular}

Table 4: Efficiencies, residual backgrounds, feed-through from radiative events into the non-radiative sample and total systematic errors of the cross-section measurements for $e^{+} e^{-} \rightarrow \tau^{+} \tau^{-}(\gamma)$ channel for different collision energies.

\begin{tabular}{|l|c|c|c|c|}
\hline \multicolumn{4}{|c|}{$e^{+} e^{-} \rightarrow \boldsymbol{q} \overline{\boldsymbol{q}}(\gamma), \sqrt{\mathrm{s}^{\prime}} / \sqrt{\mathrm{s}}>\mathbf{0 . 8 5}$} \\
\hline \multicolumn{1}{|c|}{ Energy $(\mathrm{GeV})$} & 130.2 & 136.2 & 161.3 & 172.1 \\
\hline Efficiency, \% & 85.7 & 84.6 & 87.1 & 86.9 \\
Background, \% & 1.4 & 1.5 & 5.7 & 19.7 \\
Feed-through from rad.events, \% & 18.5 & 17.4 & 11.4 & 10.2 \\
Total systematic error, \% & \pm 3.0 & \pm 3.0 & \pm 3.0 & \pm 3.0 \\
\hline
\end{tabular}

\begin{tabular}{|l|c|c|c|c|}
\hline \multicolumn{4}{|c|}{$e^{+} e^{-} \rightarrow \boldsymbol{q} \overline{\boldsymbol{q}}(\gamma), \sqrt{\mathrm{s}^{\prime}} / \sqrt{\mathrm{s}}>\mathbf{0 . 1 0}$} \\
\hline \multicolumn{1}{|c|}{ Energy $(\mathrm{GeV})$} & 130.2 & 136.2 & 161.3 & 172.1 \\
\hline \hline Efficiency, \% & 91.4 & 90.3 & 88.7 & 85.9 \\
Background, \% & 2.9 & 3.6 & 7.8 & 18.0 \\
Total systematic error, \% & \pm 0.9 & \pm 1.0 & \pm 1.1 & \pm 1.3 \\
\hline
\end{tabular}

Table 5: Efficiencies, residual backgrounds, feed-through from radiative events into the non-radiative sample and total systematic errors of the cross-section measurements for $e^{+} e^{-} \rightarrow q \bar{q}(\gamma)$ channel for different collision energies. 


\section{DELPHI}

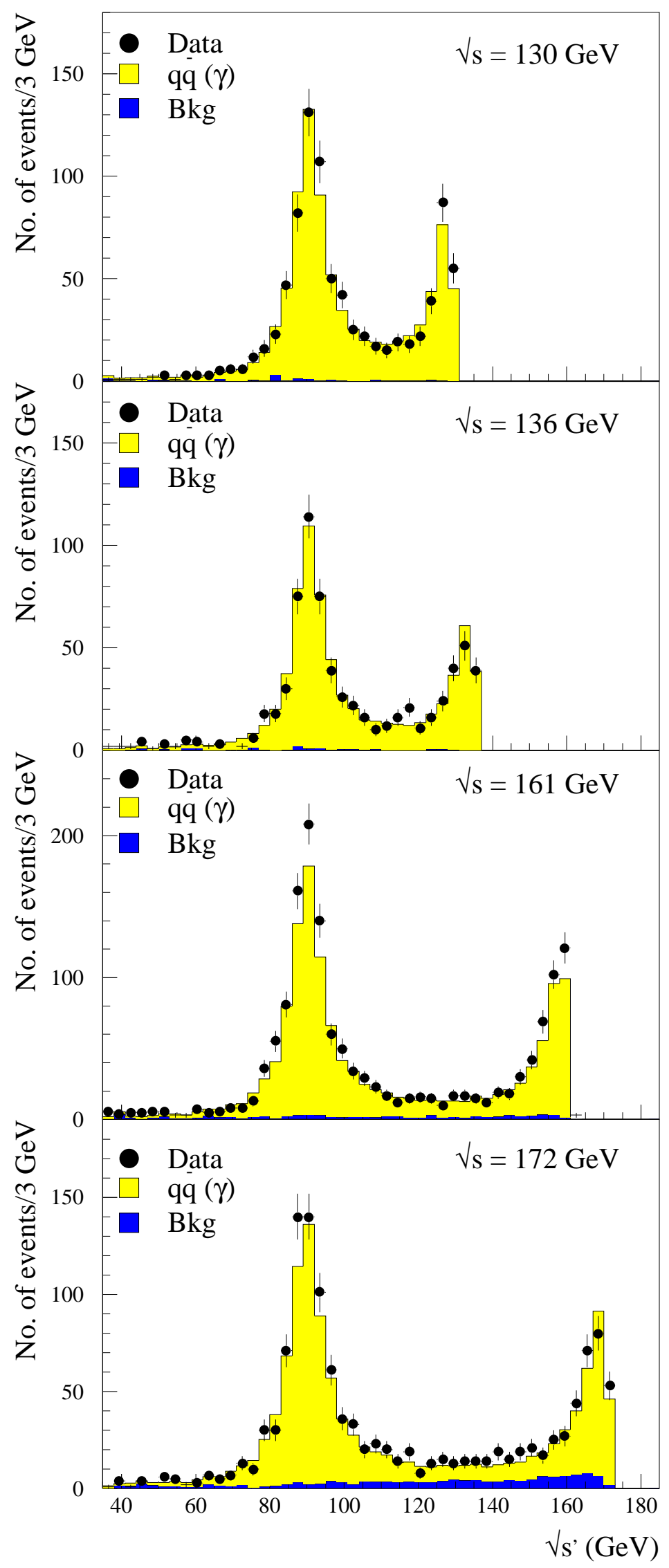

Figure 3: Distribution of the reconstructed reduced energy for the $e^{+} e^{-} \rightarrow q \bar{q}(\gamma)$ process at different collision energies. The points are the data and the histogram shows the simulation. 
cut of $5 \mathrm{GeV} / c^{2}$. To remove $W^{+} W^{-}$events in the 161 and $172 \mathrm{GeV}$ data, only events with 3 jets or less were kept. In addition, events with three jets were rejected if one of the jets contained only one charged particle and at most two neutral particles - to remove semileptonic $W$ decays. The effective centre-of-mass energy $\sqrt{s^{\prime}}$ was calculated as described in section 2.3. To remove events that return radiatively to the $Z, \sqrt{s^{\prime}} / \sqrt{s}>0.85$ was required. A total sample of 426 hadronic events at a centre-of-mass energy of 130 and $136 \mathrm{GeV}, 288$ events at $161 \mathrm{GeV}$ and 232 events at $172 \mathrm{GeV}$ were selected. The $W^{+} W^{-}$background in the inclusive hadronic event sample amounts to $0.8 \%$ at $161 \mathrm{GeV}$ and $1.7 \%$ at $172 \mathrm{GeV}$. The DYMU3 and PYTHIA $[17,15]$ generators (with DELPHI tuning [19]) were used to generate $q \bar{q}(\gamma)$ and $W^{+} W^{-}$events for the simulation.

The selected hadronic events were divided into three main classes. The first class was enriched in bottom quarks, the second in charm quarks and the third in light quarks. The flavour separation was based on the probability that all the tracks in an event came from the primary vertex [22]. For light quark events this is typically large, whereas for a large fraction of events containing a bottom quark this probability is low, due to the long lifetime of $\mathrm{B}$ hadrons. The definitions of the classes were chosen to optimise the efficiency and purity for the different categories. The efficiencies and purities after this classification for the energies of $161-172 \mathrm{GeV}$ with $\sqrt{\mathrm{s}^{\prime}} / \sqrt{\mathrm{s}}>0.85$ obtained by simulation are shown in Table 6.

Hadronic events collected to calibrate the detector at Z energies, were used to check the predicted rates observed for the 3 classes. For the 1995 data $41 \mathrm{k}$ events were selected, for the 1996 data $21 \mathrm{k}$. The fractions of tagged events in data and simulation were compared and their ratios were found to be $0.942 \pm 0.010(1.011 \pm 0.017)$ for the $b$ tag, $1.046 \pm$ $0.010(1.052 \pm 0.017)$ for the $c$ tag, and $1.006 \pm 0.005(0.983 \pm 0.010)$ for the light quark tag. The numbers in brackets correspond to the $\mathrm{Z}$ data taken in 1996, the errors are statistical only. The tagging rates agree to better than $\pm 10 \%$ with the values from the simulation. To obtain predictions for the tagging rates at $130 \mathrm{GeV}$ and above, the rates predicted by the simulation were corrected using these fractions. It was assumed that the fractions of bottom, charm and light quark events at the $\mathrm{Z}$ are the same as predicted in the Standard Model. Potential errors arising from the corrections applied to the high energy data, but calculated at the $\mathrm{Z}$ due to differences in properties of the events (for example, the flavour composition which could give different biases through the purity) are included in the systematic error.

The observed numbers of events in the flavour-tagged samples of hadronic final states were compared to the expected number of events assuming the Standard Model fractions for bottom, charm and light quark events, after applying the correction factors obtained from the $\mathrm{Z}$ data. The results for the $\mathrm{b}, \mathrm{c}$ and $\mathrm{uds}$ enriched classes are shown in Table 7 , together with the systematic and statistical errors coming from the simulation. The systematic error had two components. The first was due to the statistical uncertainty on the correction derived from the normalisation of the quark production rates to the hadronic $Z$ data. The second component was an estimate of the uncertainty in the bottom, charm and light quark efficiencies which was taken from the changes in tagging efficiencies in simulation which would be needed to obtain the tagging rates in data at the Z. These changes in efficiency were then applied at the higher energies and the differences in the number of events tagged in the simulation were assigned as the systematic error, taken to cover possible errors due to differences between events at the $Z$ and at higher energies. The error on the expected tagging rate was then evaluated by taking the worst case scenarios, e.g. where discrepancies for the light quark tag were fully attributed to presence of charm background. The quadratic sum of these two components is quoted as 


\begin{tabular}{|c|c|c|c|c|}
\hline tag & efficiency & b content & c content & uds content \\
\hline \hline b enriched & $0.78(\mathrm{~b})$ & 0.76 & 0.19 & 0.05 \\
\hline c enriched & $0.35(\mathrm{c})$ & 0.14 & 0.43 & 0.43 \\
\hline uds enriched & $0.79(\mathrm{uds})$ & 0.03 & 0.21 & 0.76 \\
\hline
\end{tabular}

Table 6: Efficiencies and purities for the different tags at energies of $161-172 \mathrm{GeV}$.

the total systematic error. The results for the observed number of events were compatible within approximately one standard deviation with the number of expected events in the Standard Model.

At the Z-pole the forward-backward asymmetries for quarks are around $10 \%$ in the Standard Model. At centre-of-mass energies of 161-172 GeV, much larger forwardbackward asymmetries of typically $50 \%$ and higher are expected. The hemisphere charge $Q_{\text {hemi }}[23]$ was used to determine the direction of the quark and that of the anti-quark,

$$
Q_{h e m i}=\sum_{i} q_{i} p_{i / /}^{\kappa} / \sum_{i} p_{i / /}^{\kappa}
$$

with $\kappa=0.6, p_{i / /}$ the momentum component along the thrust axis, and $i$ runs over the charged particles in one hemisphere, defined by the thrust axis. The forward-backward hemisphere charge $Q_{F B}$ is defined as the difference of the charges in the two hemispheres. Events with a $\left|Q_{F B}\right|>0.2$ were selected, and the direction of the thrust axis was signed, assuming the quark (not anti-quark) had positive charge, to give an estimate of the initial quark direction. The angular distribution can be described by:

$$
\frac{d \sigma}{d \cos \theta_{t}}=1+\cos ^{2} \theta_{t}+8 / 3 A_{F B} \cos \theta_{t},
$$

where $\theta_{t}$ is the signed polar angle of the thrust axis and $A_{F B}$ the forward-backward charge asymmetry. The angular distributions were fitted in the range $\left|\cos \theta_{t}\right|<0.8$. The charge asymmetry is positive for charm and up quarks, and negative for bottom, strange and down quarks. Angular dependent efficiency effects were negligible at the level of precision of this measurement.

The observed asymmetry $A_{F B}^{o b s}$ is smaller than the real asymmetry because the hemisphere charge sometimes gives the wrong sign. This can be expressed by a charge confusion factor $C$, according to the following equation:

$$
A_{F B}^{o b s}=C A_{F B} .
$$

Using the simulation, the constant $C$ was determined to be 0.54 for bottom, 0.37 for charm, 0.55 for strange, 0.62 for up, and 0.52 for down quarks at energies of 161-172 $\mathrm{GeV}$. The uncertainty on the charge confusion factors determined in this way was $15 \%$ The angular distributions at energies of $161-172 \mathrm{GeV}$ for all events and for bottom, charm and light quark enriched samples are shown together with the fitted curves in Figure 4. The shaded areas give the Standard Model predictions from the simulation.

The results for the different samples at energies of 130-136 and 161-172 GeV for the observed and expected charge asymmetry are shown in Table 8.

The systematic error listed in the last column comes from two sources: firstly, from the uncertainty on the charge confusion factors $C$ and secondly from the discrepancies 


\begin{tabular}{|c|c|c|c|c|}
\hline \multicolumn{5}{|c|}{$\sqrt{s}=130-136 \mathbf{G e V}$} \\
\hline tag & $\begin{array}{c}\text { observed } \\
\text { events }\end{array}$ & $\begin{array}{c}\text { expected } \\
\text { events }\end{array}$ & $\begin{array}{c}\text { stat.error } \\
(\mathrm{sim})\end{array}$ & $\begin{array}{c}\text { syst.error } \\
(\mathrm{sim})\end{array}$ \\
\hline \hline b enriched & 68 & 70.4 & \pm 2.7 & \pm 0.9 \\
\hline c enriched & 73 & 76.1 & \pm 2.8 & \pm 1.3 \\
\hline uds enriched & 251 & 245.5 & \pm 3.3 & \pm 2.0 \\
\hline
\end{tabular}

\begin{tabular}{|c|c|c|c|c|}
\hline \multicolumn{5}{|c|}{$\sqrt{s}=\mathbf{1 6 1}-\mathbf{1 7 2} \mathbf{G e V}$} \\
\hline tag & $\begin{array}{c}\text { observed } \\
\text { events }\end{array}$ & $\begin{array}{c}\text { expected } \\
\text { events }\end{array}$ & $\begin{array}{c}\text { stat.error } \\
(\mathrm{sim})\end{array}$ & $\begin{array}{c}\text { syst.error } \\
(\mathrm{sim})\end{array}$ \\
\hline \hline b enriched & 85 & 95.2 & \pm 1.3 & \pm 2.1 \\
\hline c enriched & 117 & 105.3 & \pm 1.4 & \pm 2.2 \\
\hline uds enriched & 294 & 295.5 & \pm 1.6 & \pm 3.3 \\
\hline
\end{tabular}

Table 7: The observed and expected numbers of events for the different tags at energies of 130-136 and $161-172 \mathrm{GeV}$ with $\sqrt{\mathrm{s}^{\prime}} / \sqrt{\mathrm{s}}>0.85$.

\begin{tabular}{|c|c|c|c|c|}
\hline \multicolumn{5}{|c|}{$\sqrt{\boldsymbol{s}}=\mathbf{1 3 0}-\mathbf{1 3 6} \mathbf{G e V}$} \\
\hline tag & $A_{F B}^{\text {obs }}$ & $A_{F B}^{\exp }$ & $\begin{array}{c}\text { stat.error } \\
(\mathrm{sim})\end{array}$ & $\begin{array}{c}\text { syst.error } \\
(\mathrm{sim})\end{array}$ \\
\hline \hline all & $-0.039 \pm 0.065$ & 0.001 & \pm 0.023 & \pm 0.002 \\
\hline b enriched & $-0.199 \pm 0.153$ & -0.113 & \pm 0.059 & \pm 0.020 \\
\hline c enriched & $0.078 \pm 0.146$ & 0.048 & \pm 0.058 & \pm 0.010 \\
\hline uds enriched & $-0.044 \pm 0.086$ & 0.021 & \pm 0.031 & \pm 0.010 \\
\hline
\end{tabular}

\begin{tabular}{|c|c|c|c|c|}
\hline \multicolumn{5}{|c|}{$\sqrt{s}=161-172 \mathrm{GeV}$} \\
\hline $\operatorname{tag}$ & $A_{F B}^{o b s}$ & $A_{F B}^{e x p}$ & $\begin{array}{l}\text { stat.error } \\
(\operatorname{sim})\end{array}$ & $\begin{array}{l}\text { syst.error } \\
(\operatorname{sim})\end{array}$ \\
\hline all & $0.025 \pm 0.058$ & 0.023 & \pm 0.009 & \pm 0.002 \\
\hline b enriched & $-0.357 \pm 0.139$ & -0.146 & \pm 0.023 & \pm 0.020 \\
\hline c enriched & $0.120 \pm 0.127$ & 0.044 & \pm 0.023 & \pm 0.010 \\
\hline uds enriched & $0.072 \pm 0.076$ & 0.064 & \pm 0.013 & \pm 0.010 \\
\hline
\end{tabular}

Table 8: The observed and expected forward-backward charge asymmetries for the different tags at energies of 130-136 and 161-172 GeV with $\sqrt{\mathrm{s}^{\prime}} / \sqrt{\mathrm{s}}>0.85$. 

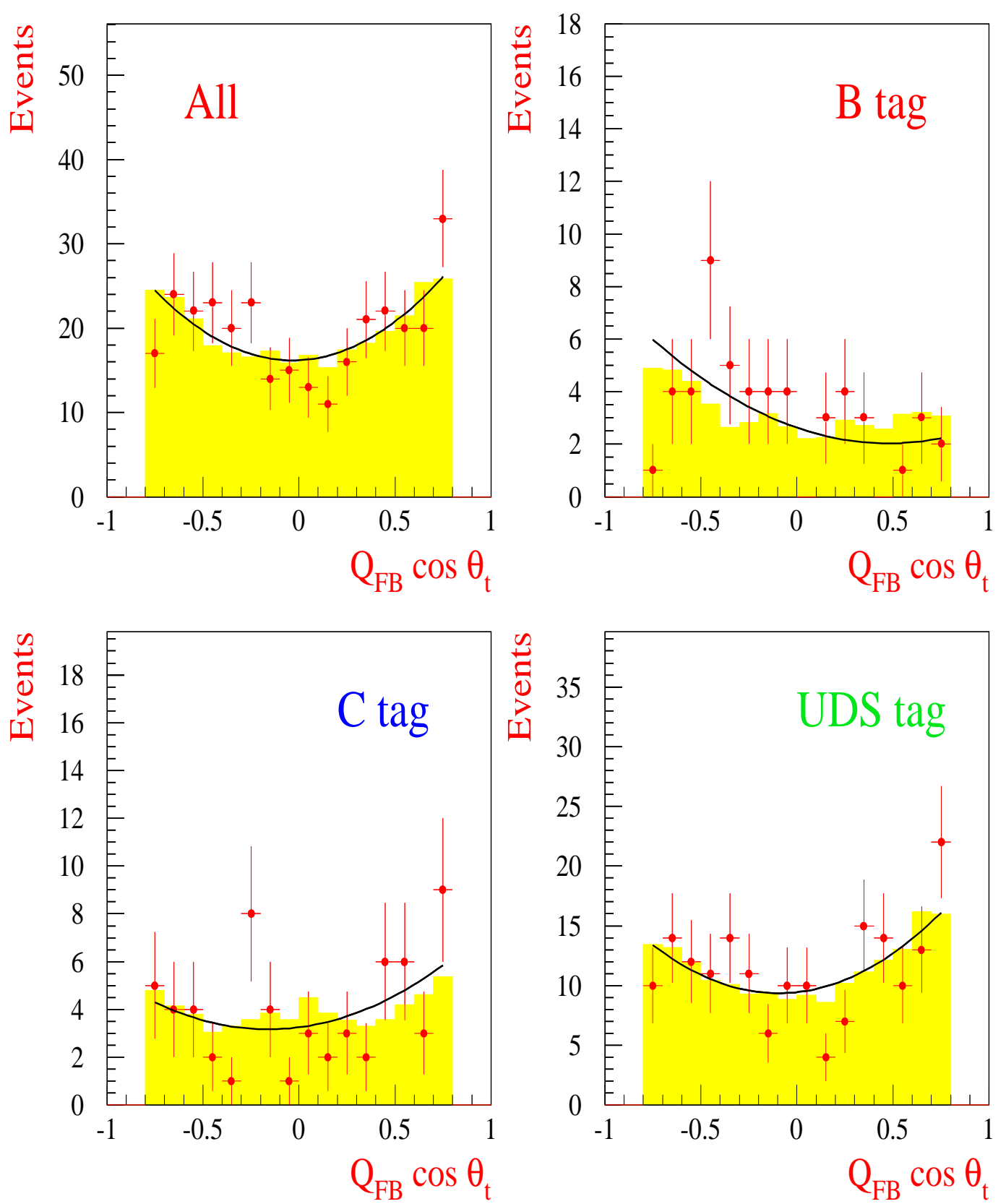

Figure 4: The charge-signed polar angle distributions for all events, b, $c$ and uds enriched events at energies of 161-172 GeV. The shaded area gives the Standard Model prediction, normalised to the data, the full curve shows the fit of equation 4 to the data. 


\begin{tabular}{|c|c|c|c|c|c|}
\hline \multicolumn{2}{|c|}{ Energy $(\mathrm{GeV})$} & 130.2 & 136.2 & 161.3 & 172.1 \\
\hline \hline \multicolumn{2}{|c|}{ Integrated Luminosity $\left(\mathrm{pb}^{-1}\right)$} & 2.87 & 2.96 & 10.09 & 10.12 \\
\hline \hline Number of events: & $e^{+} e^{-} \rightarrow q \bar{q}(\gamma)$ & 868 & 715 & 1526 & 1288 \\
& $e^{+} e^{-} \rightarrow e^{+} e^{-}(\gamma)$ & 129 & 152 & 276 & 312 \\
& $e^{+} e^{-} \rightarrow \mu^{+} \mu^{-}(\gamma)$ & 56 & 40 & 72 & 70 \\
& $e^{+} e^{-} \rightarrow \tau^{+} \tau^{-}(\gamma)$ & 33 & 29 & 58 & 54 \\
\hline
\end{tabular}

Table 9: Integrated luminosity and statistics used in the analyses of the different final states and collision energies. For the $e^{+} e^{-} \rightarrow e^{+} e^{-}(\gamma)$ channel, the values refer to the analysis in the central angular region with $\theta_{\text {acol }}<90^{\circ}$, whereas for the other channels, the numbers refer to the analyses with low s' cut.

between data and simulation observed in the tagging rates at the $\mathrm{Z}$ which were propagated according to the method described above.

The results for the observed forward-backward charge asymmetries are in agreement with the Standard Model expectation.

Possible detector effects affecting the asymmetry measurement were studied. Several distributions were checked using the higher statistics $\mathrm{Z}$ data, e.g. by comparing the hemisphere charge in the forward region and the $s^{\prime}$ distributions with the simulation. The distributions were consistent with the expectations from the simulation. Further, the observed forward-backward charge asymmetries at the $\mathrm{Z}$ were compared to the Standard Model expectations for flavour-tagged events and found to be $A_{F B}^{o b s}-A_{F B}^{e x p}=-0.015 \pm$ 0.013 (all), $-0.003 \pm 0.036$ (b enriched), $-0.013 \pm 0.034$ (c enriched) and $-0.023 \pm 0.019$ (uds enriched); consistent with expectations.

\section{Results for cross-sections and asymmetries}

\subsection{Inclusive $e^{+} e^{-} \rightarrow q \bar{q}(\gamma)$ and leptonic final states}

The luminosity and statistics accumulated at each collision energy are summarised in Table 9 for the inclusive $e^{+} e^{-} \rightarrow q \bar{q}(\gamma)$ final state and leptonic final states. The results of the cross-section and asymmetry measurements for these different final states are summarised in Table 10. The errors indicated are statistical only. The systematic errors are those presented in Table 1,3,4,5 and in the section devoted to the luminosity measurement.

For the $e^{+} e^{-}$final state the photon exchange in the $t$-channel dominates the measured cross-section. As a consequence, the $s$-channel contribution cannot be extracted reliably. These $e^{+} e^{-}$cross-sections and asymmetries were not included in the S-matrix fits described in Section 4. However, as can be seen from Table 10, they are compatible with the Standard Model predictions calculated with the TOPAZ0 program [24].

Figures 5 and 6 show the measured hadron, muon and tau cross-sections and forwardbackward asymmetries from the $Z^{0}$-peak energies up to $172 \mathrm{GeV}$. The muon data below the $Z^{0}$, shown in the same figures, are taken from [25]. The electron cross-section and forward-backward asymmetry including full $(s+t)$ contribution are presented in Figures 7 and 8 . The curves show the ZFITTER, or TOPAZ0 (in the case of electrons), predictions. 


\begin{tabular}{|c|c|c|c|c|c|}
\hline \multicolumn{2}{|c|}{ Energy $(\mathrm{GeV})$} & 130.2 & 136.2 & 161.3 & 172.1 \\
\hline \hline$\sigma_{\text {had }}(p b)$ & $\sqrt{\mathrm{s}^{\prime}} / \sqrt{\mathrm{s}}>0.85$ & $82.1 \pm 5.2$ & $65.1 \pm 4.7$ & $40.9 \pm 2.1$ & $30.3 \pm 1.9$ \\
& $\mathrm{SM}$ & 83.1 & 67.0 & 34.8 & 28.9 \\
& $\sqrt{\mathrm{s}^{\prime}} / \sqrt{\mathrm{s}}>0.10$ & $328.4 \pm 11.4$ & $259.6 \pm 10.0$ & $158.3 \pm 4.5$ & $125.5 \pm 4.2$ \\
& $\mathrm{SM}$ & 327.2 & 270.5 & 147.0 & 123.0 \\
\hline$\sigma_{\mu \mu}(p b)$ & $\sqrt{\mathrm{s}^{\prime}} / \sqrt{\mathrm{s}}>0.85$ & $9.7 \pm 1.9$ & $6.6 \pm 1.6$ & $3.6 \pm 0.7$ & $3.6 \pm 0.7$ \\
& $\mathrm{SM}$ & 8.1 & 7.0 & 4.5 & 3.8 \\
& $\sqrt{s^{\prime}}>75 \mathrm{GeV}$ & $24.3 \pm 3.2$ & $17.0 \pm 2.6$ & $9.3 \pm 1.1$ & $8.9 \pm 1.1$ \\
& $\mathrm{SM}$ & 19.9 & 17.0 & 10.2 & 8.7 \\
\hline \multirow{2}{*}{$\sigma_{\tau \tau}(p b)$} & $\sqrt{\mathrm{s}^{\prime}} / \sqrt{\mathrm{s}}>0.85$ & $10.2 \pm 3.1$ & $8.8 \pm 3.0$ & $5.1 \pm 1.2$ & $4.5 \pm 1.1$ \\
& $\mathrm{SM}$ & 8.3 & 7.2 & 4.6 & 3.9 \\
& $\sqrt{s^{\prime}}>75 \mathrm{GeV}$ & $22.2 \pm 4.6$ & $17.7 \pm 3.9$ & $11.7 \pm 1.8$ & $11.2 \pm 1.8$ \\
& $\mathrm{SM}$ & 20.2 & 17.2 & 10.3 & 8.8 \\
\hline \hline \multirow{4}{*}{$A_{\mathrm{FB}}^{\mu}$} & $\sqrt{\mathrm{s}^{\prime}} / \sqrt{\mathrm{s}}>0.85$ & $0.67 \pm 0.15$ & $0.74 \pm 0.16$ & $0.43 \pm 0.16$ & $0.94 \pm 0.14$ \\
& $\mathrm{SM}$ & 0.72 & 0.69 & 0.62 & 0.61 \\
& $\sqrt{s^{\prime}}>75 \mathrm{GeV}$ & $0.45 \pm 0.12$ & $0.56 \pm 0.13$ & $0.39 \pm 0.11$ & $0.55 \pm 0.10$ \\
& $\mathrm{SM}$ & 0.34 & 0.34 & 0.33 & 0.33 \\
\hline$A_{\mathrm{FB}}^{\tau}$ & $\sqrt{\mathrm{s}^{\prime}} / \sqrt{\mathrm{s}}>0.85$ & $0.73 \pm 0.17$ & $0.49 \pm 0.23$ & $0.92 \pm 0.08$ & $0.13 \pm 0.20$ \\
& $\mathrm{SM}$ & 0.72 & 0.70 & 0.62 & 0.61 \\
& $\sqrt{s^{\prime}}>75 \mathrm{GeV}$ & $0.31 \pm 0.17$ & $0.26 \pm 0.19$ & $0.39 \pm 0.12$ & $0.19 \pm 0.14$ \\
& $\mathrm{SM}$ & 0.33 & 0.33 & 0.32 & 0.32 \\
\hline \hline \multirow{2}{*}{$\sigma_{e e}^{e}(p b)$} & $\theta_{a c o l}<20^{\circ}$ & $42.0 \pm 4.0$ & $47.1 \pm 4.2$ & $27.1 \pm 1.8$ & $30.3 \pm 1.9$ \\
& $\mathrm{SM}$ & 48.7 & 44.6 & 31.9 & 28.0 \\
& $\theta_{a c o l}<90^{\circ}$ & $48.0 \pm 4.3$ & $54.1 \pm 4.5$ & $30.7 \pm 1.9$ & $33.7 \pm 2.0$ \\
& $\mathrm{SM}$ & 56.3 & 50.8 & 35.1 & 30.6 \\
\hline & $\theta_{a c o l}<20^{\circ}$ & $0.81 \pm 0.06$ & $0.89 \pm 0.04$ & $0.82 \pm 0.04$ & $0.81 \pm 0.04$ \\
& $\mathrm{SM}$ & 0.81 & 0.81 & 0.82 & 0.82 \\
& & $0.75 \pm 0.06$ & $0.78 \pm 0.05$ & $0.77 \pm 0.04$ & $0.76 \pm 0.04$ \\
& & 0.72 & 0.73 & 0.76 & 0.77 \\
\hline
\end{tabular}

Table 10: Results of the cross-section and asymmetry measurements for the different final states and collision energies. The errors indicated are statistical only. Systematic errors are given in Tables 1,3,4 and 5 and in the section devoted to the luminosity measurement. The Standard Model predictions of ZFITTER and for the $e^{+} e^{-}$channel TOPAZ0 are also indicated. The hadronic, muon and tau results are corrected for all cuts, apart from the $s^{\prime}$ cut. In case of the $e^{+} e^{-} \rightarrow e^{+} e^{-}(\gamma)$ channel the numbers are restricted to the barrel analysis, and are corrected for all cuts except the acollinearity and polar angle acceptance. 


\section{DELPHI}

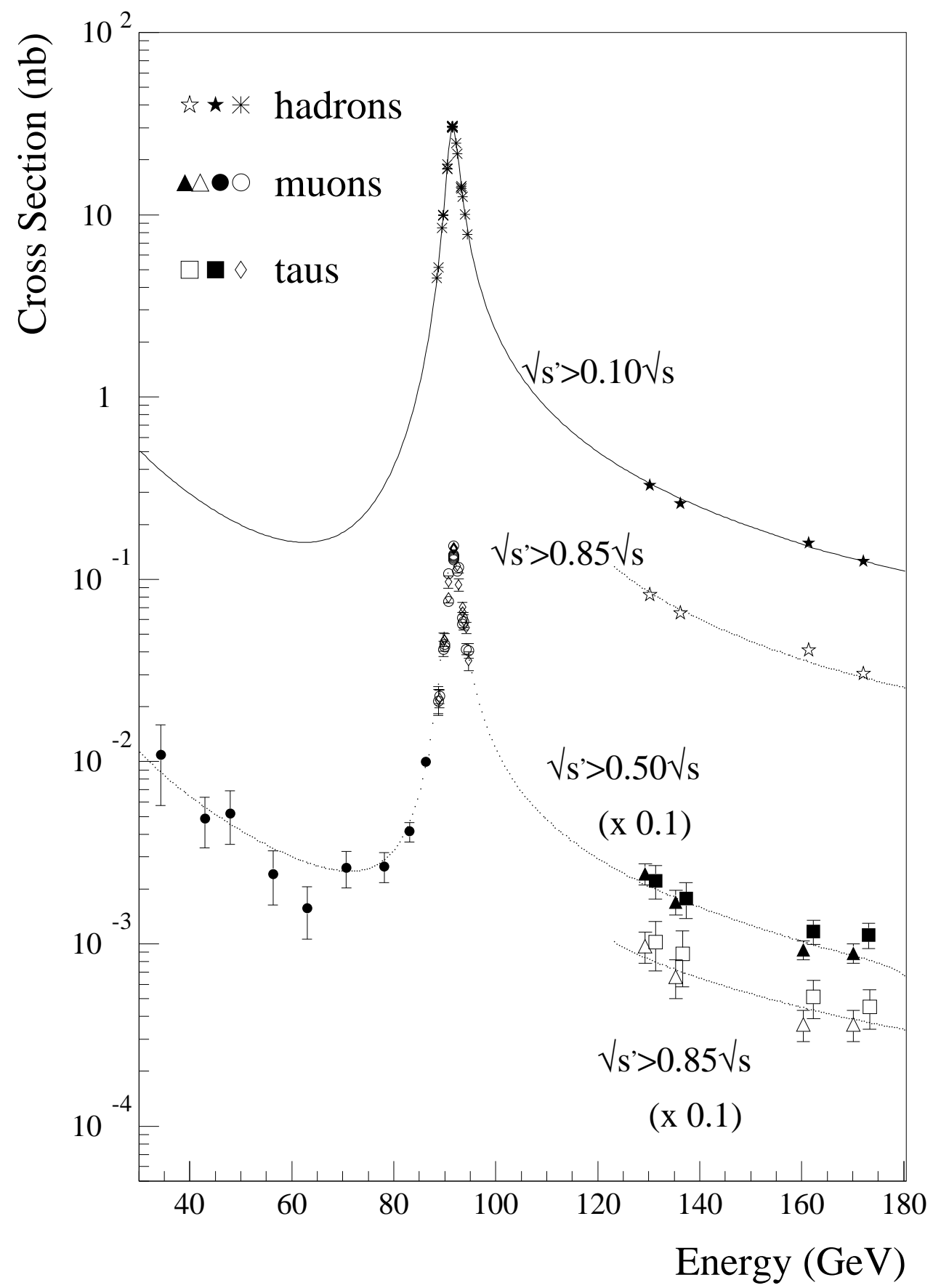

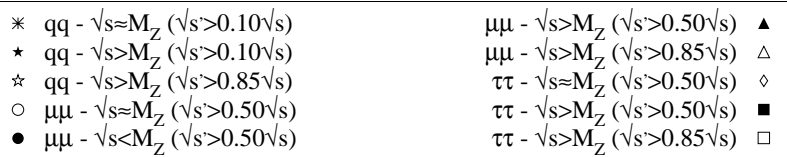

Figure 5: Cross-sections for $e^{+} e^{-} \rightarrow q \bar{q}(\gamma), \mu^{+} \mu^{-} \gamma$ and $\tau^{+} \tau^{-} \gamma$ processes measured from $Z^{0}$-resonance energies up to $172 \mathrm{GeV}$. The data at the $\mathrm{Z}$ resonance are the published results of the $\mathrm{Z}$ lineshape corrected to the acceptance $\sqrt{s^{\prime}}>0.10 \sqrt{s}$ for hadrons and $\sqrt{s^{\prime}}>0.50 \sqrt{s}$ for leptons. The data for muons below the $\mathrm{Z}$ peak are from the analysis of $e^{+} e^{-} \rightarrow \mu^{+} \mu^{-}(\gamma)$ data at LEP I, also corrected to $\sqrt{s^{\prime}}>0.50 \sqrt{s}$. The curves are the predictions of the ZFITTER program. 


\section{DELPHI}

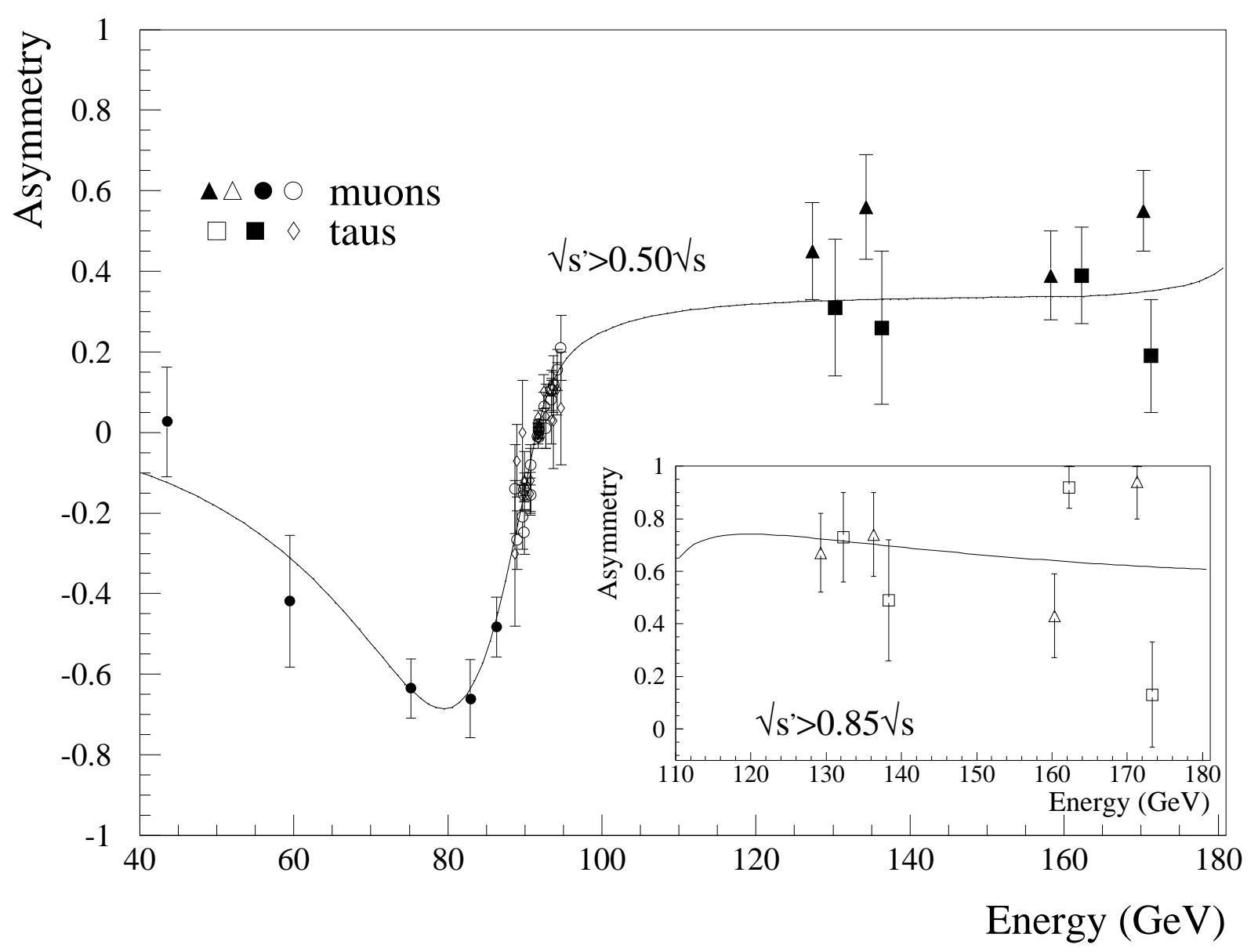

$\mu \mu-\sqrt{s}_{\mathrm{s}} \approx \mathrm{M}_{\mathrm{Z}}\left(\sqrt{ } \mathrm{s}^{\prime}>0.50 \sqrt{ } \mathrm{s}\right)$

- $\mu \mu-\sqrt{s}_{\mathrm{s}}<\mathrm{M}_{\mathrm{Z}}\left(\sqrt{ }_{\mathrm{s}}>0.50 \sqrt{ }_{\mathrm{s}}\right)$

$\tau \tau-\sqrt{s} \approx M_{Z}\left(\sqrt{ } s^{\prime}>0.50 \sqrt{ } s\right)$

$\tau \tau-\sqrt{s}>M_{Z}\left(\sqrt{ } s^{\prime}>0.50 \sqrt{ }\right)$

$\Delta \mu \mu-\sqrt{s}_{\mathrm{s}}>\mathrm{M}_{\mathrm{Z}}\left(\sqrt{ } \mathrm{s}^{\prime}>0.50 \sqrt{ }_{\mathrm{s}}\right)$

$\triangle \mu \mu-\sqrt{s}_{\mathrm{s}}>\mathrm{M}_{\mathrm{Z}}\left(\sqrt{ }^{\prime}>0.85 \sqrt{ } \mathrm{s}\right)$

Figure 6: The forward-backward charge asymmetries measured in the reactions $e^{+} e^{-} \rightarrow \mu^{+} \mu^{-}(\gamma)$ and $\tau^{+} \tau^{-} \gamma$ at energies from $Z^{0}$-peak up to $172 \mathrm{GeV}$. The data at the $\mathrm{Z}$ resonance are the published results of the $\mathrm{Z}$ lineshape corrected to the acceptance $\sqrt{s^{\prime}}>0.10 \sqrt{s}$ for hadrons and $\sqrt{s^{\prime}}>0.50 \sqrt{s}$ for leptons. The data for muons below the $\mathrm{Z}$ peak are from the analysis of $e^{+} e^{-} \rightarrow \mu^{+} \mu^{-}(\gamma)$ data at LEP I, also corrected to $\sqrt{s^{\prime}}>0.50 \sqrt{s}$. The insert shows the data with the cut $\sqrt{s^{\prime}}>0.85 \sqrt{s}$ The curves are the predictions of the ZFITTER program. 


\section{DELPHI}

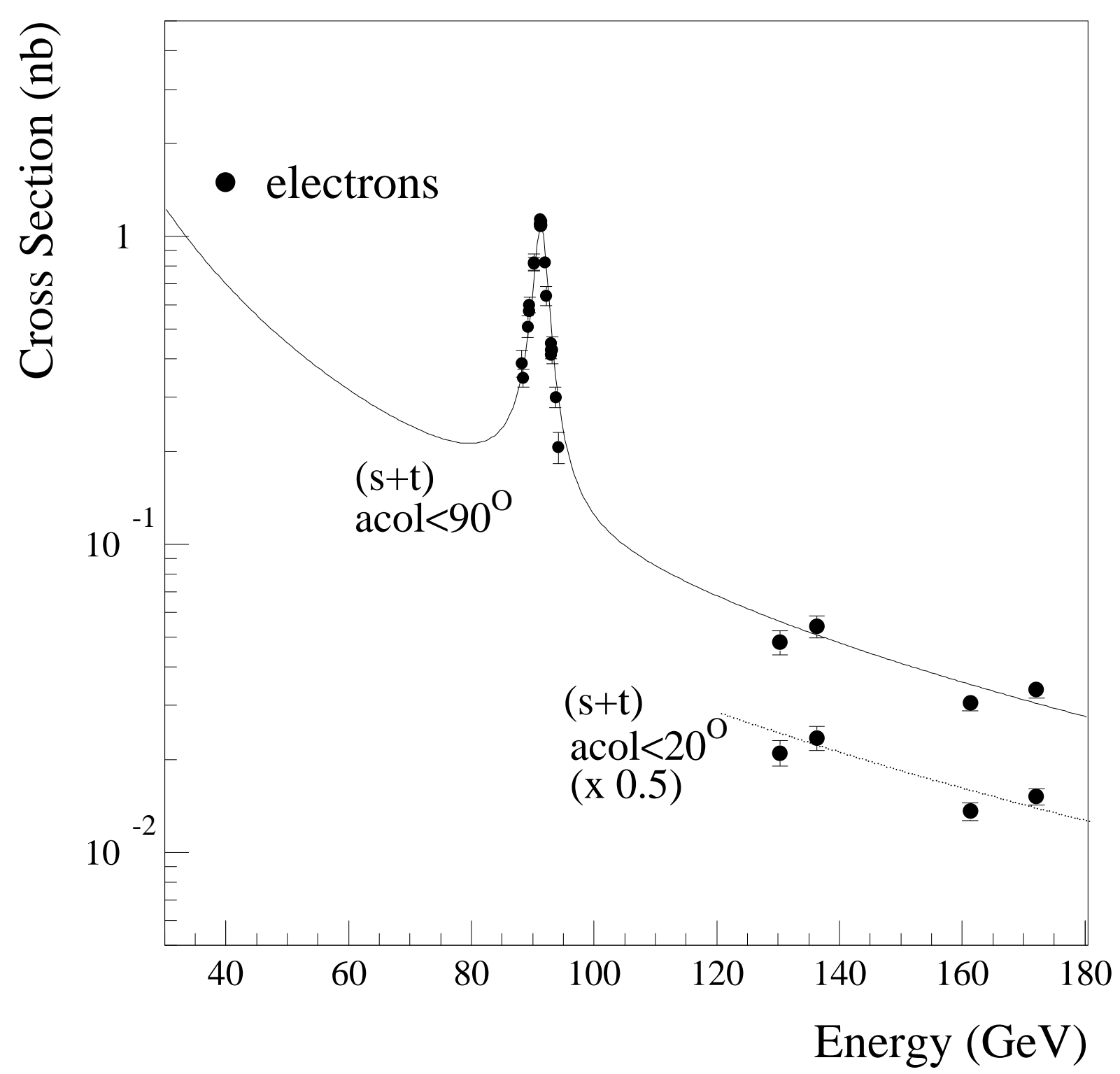

Figure 7: The measured cross-sections for $e^{+} e^{-} \rightarrow e^{+} e^{-}(\gamma)$ from the $Z^{0}$-peak energies up to $172 \mathrm{GeV}$. The curves are the predictions of the TOPAZ0 program. 


\section{DELPHI}

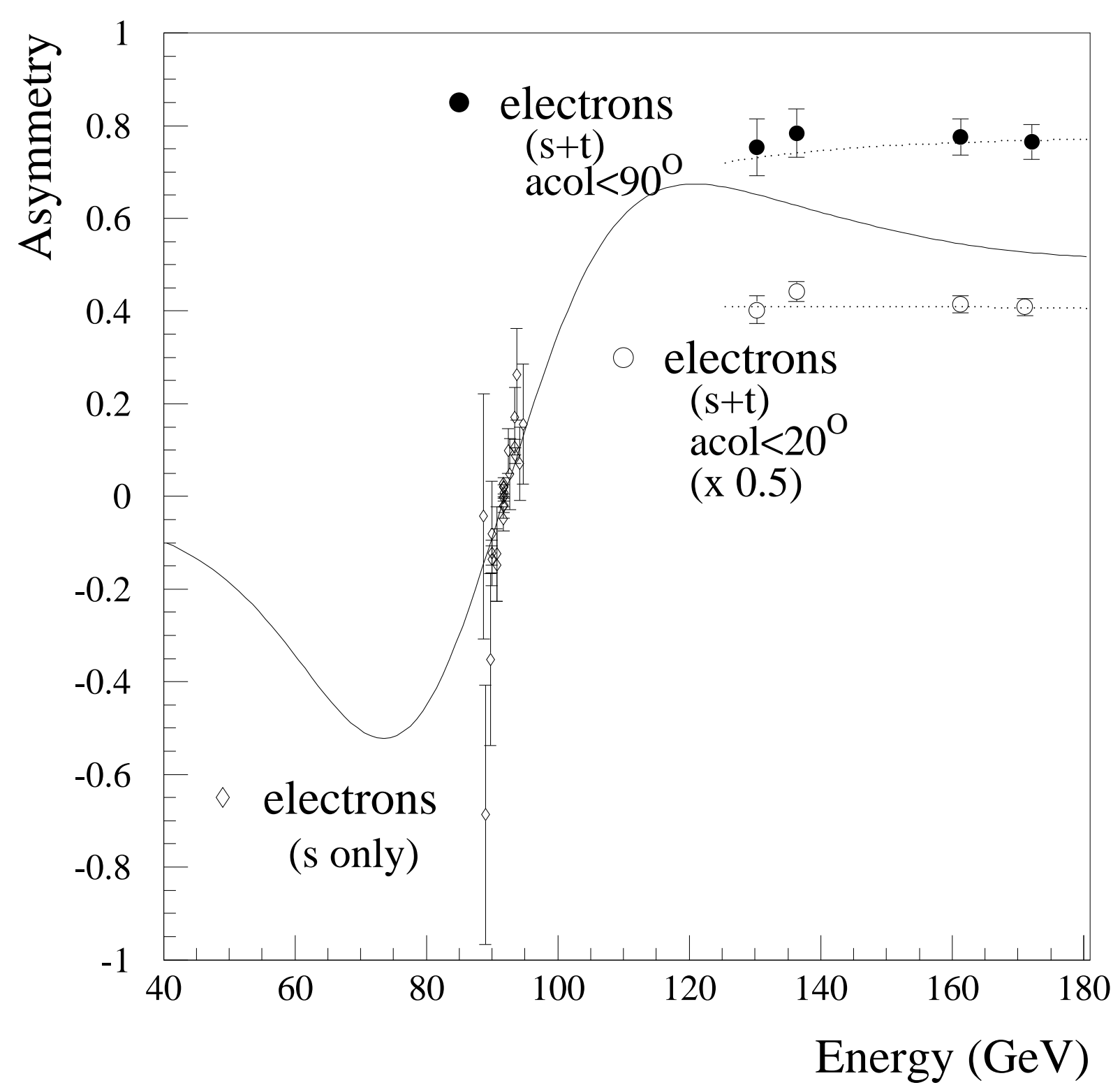

Figure 8: The measured forward-backward charge asymmetries for $e^{+} e^{-} \rightarrow e^{+} e^{-}(\gamma)$ from the $Z^{0}$-peak energies up to $172 \mathrm{GeV}$. The curves are the predictions of the TOPAZ0 program. 


\begin{tabular}{|c|c|c|}
\hline $\begin{array}{c}\text { Collision energy } \\
(\mathrm{GeV})\end{array}$ & $\begin{array}{c}\text { Total number } \\
\text { of events }\end{array}$ & $\begin{array}{c}\text { Cross-section } \\
(\mathrm{nb})\end{array}$ \\
\hline \hline 130.2 & 2697 & $1.125 \pm 0.022$ \\
136.2 & 2585 & $1.032 \pm 0.020$ \\
161.3 & 6055 & $0.776 \pm 0.010$ \\
172.1 & 5546 & $0.695 \pm 0.009$ \\
\hline
\end{tabular}

Table 11: The number of events and the integrated cross-section in the range $12^{\circ}<\theta<35^{\circ}$ for the reaction $e^{+} e^{-} \rightarrow e^{+} e^{-}(\gamma)$ as a function of collision energy. The errors given are statistical only.

\section{$3.2 \quad$ Forward $e^{+} e^{-} \rightarrow e^{+} e^{-}(\gamma)$}

The differential cross-sections for forward $e^{+} e^{-} \rightarrow e^{+} e^{-}(\gamma)$ measured at collision energies of 130 to $172 \mathrm{GeV}$ are shown in Figure 9 compared to the predictions of ALIBABA [26]. The total numbers of events selected and the cross-sections integrated over the full angular coverage are given in Table 11 . The systematic error on these measurements was estimated to be $\pm 2.6 \%$, with a dominant contribution from the knowledge of the acceptance, the precision of the absolute polar angle calibration being $\pm 0.13^{\circ}$. The data are in agreement with the theoretical predictions.

\subsection{Flavour-tagged events}

From the measured event rates and asymmetries for flavour-tagged events it is possible to extract the ratios of cross-sections and asymmetries for bottom, charm, strange, up and down quarks. The cross-section ratio $R_{q}$ is defined as the ratio of the quark cross-section $\sigma_{q}$ and the total hadronic cross-section $\sigma_{h}$.

To extract the cross-section for one quark flavour, the cross-sections for the other flavours were taken from the Standard $\mathrm{Model}^{2}$. The results are given in Table 12. The central values of the derived ratios of quark production cross-sections compared to all hadrons do not add up exactly to unity. This is because of the various correction factors, taken from data and simulation, applied to the observed numbers in the three tagged samples to obtain the quark production rates. In Figure 10, the extracted values of $R_{q}$ for bottom, charm and light uds quarks are shown as a function of the centre-of-mass energy, together with the predictions from ZFITTER.

To extract the forward-backward asymmetry for one quark flavour, the cross-sections and asymmetries for the other flavours were taken from the Standard Model. The observed asymmetry $A_{F B}^{o b s}$ is related to the quark asymmetries $A_{F B}^{i}$ in the following way:

$$
A_{F B}^{o b s}=\sum_{i} \frac{q_{i}}{\left|q_{i}\right|} C_{i} P_{i} A_{F B}^{i},
$$

where $i$ runs over the quarks, $C$ denotes the charge confusion factor, and $P$ the purity, and $q_{i}$ the charge on the quark. This definition results in a minus sign to convert the observed charge asymmetry into the forward-backward quark asymmetry for bottom, strange and

\footnotetext{
${ }^{2}$ According to the formula $R_{q}=\frac{\sigma_{q}}{\sigma_{h}}=R_{q}^{S M}\left(1+\frac{N_{q}^{o b s}-N^{e x p}}{N^{e x p} P_{q}}\right)$, where $q$ denotes b, c or uds quarks, $R_{q}$ refers to the extracted cross-section ratio, $R_{q}^{S M}$ to the Standard Model expectation, $N_{q}^{o b s}$ ( $N_{q}^{e x p}$ ) to the observed (expected) number of events for a given $q$ enriched tag (see Table 7 ) and $P_{q}$ to the $q$ purity for a $q$ enriched tag. For the $161-172$ GeV data the purities can be found in Table 6 .
} 


\section{DELPHI}
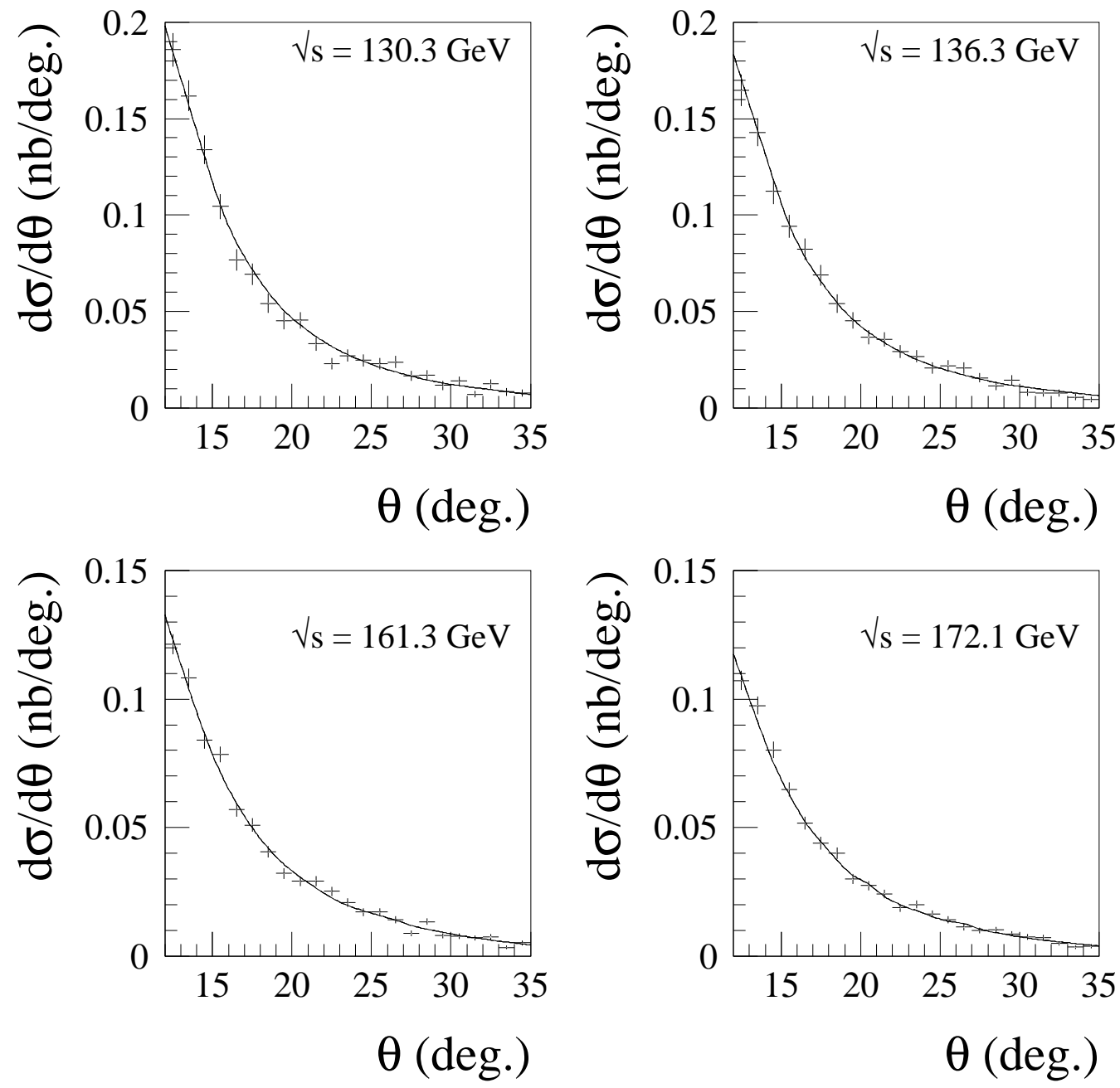

Figure 9: Differential cross-sections for the process $e^{+} e^{-} \rightarrow e^{+} e^{-}(\gamma)$ in the forward region for collision energies of 161 and $172 \mathrm{GeV}$. The data are shown by the points and the curves are theoretical predictions computed using the ALIBABA program. 
down quarks, which corrects the implicit assumption that the charge of the quark was positive, which was introduced when signing the thrust axis by the forward-backward hemisphere-charge. The results of this procedure and the correlations ${ }^{3}$ are given in Table 12. The forward-backward quark asymmetries should by definition lie between -1 and 1 , the measured quark asymmetry can go outside this range because the charge confusion factor and the purity are both smaller than 1. The derived up, down and strange quark asymmetries are fully correlated because they are derived from the charge asymmetry measured in the uds enriched sample. The DELPHI data confirm the expected rise of the forward-backward asymmetry for quarks as a function of the centre-of-mass energy above the Z-pole.

The measurements for flavour-tagged cross-section ratios and asymmetries are in agreement with the Standard Model.

\section{Interpretation of results using S-matrix formalism}

In this section the results of the inclusive $q \bar{q}$ cross-sections and the leptonic crosssections and forward-backward asymmetries are discussed in the context of the Standard Model. As can be seen from Table 10 the results are in reasonable agreement with the expectations of the Standard Model.

The underlying physics can be parameterised in a quasi-model independent way using the S-matrix approach $[27,28]$. Fits to the measured inclusive hadronic, muon and tau cross-sections and muon and tau forward-backward asymmetries were carried out in this framework using the corresponding branch of the ZFITTER program. The fits included also hadronic, electron, muon and tau data collected by DELPHI near the $Z^{0}$ resonance $[1,2]$. The usual definitions of the mass $\left(M_{\mathrm{Z}}\right)$ and width $\left(\Gamma_{\mathrm{Z}}\right)$ of a Breit-Wigner resonance were used, the width being $s$-dependent.

The S-matrix parameters $r$ and $\mathrm{j}$ scale the $Z^{0}$ exchange and the $\gamma Z^{0}$ interference contributions to the total cross-section and forward-backward lepton asymmetries. The contribution of the pure $\gamma$ exchange was fixed to its value predicted by QED in all fits.

The results of the fits are presented in Table 13. The $\chi^{2}$ amounted to 227.9 in the case of the 16-parameter fit (i.e. without assuming lepton universality) and to 237.1 for the 8 -parameter fit (where lepton universality was assumed). The number of points fitted was 217 in both cases. The correlation coefficients between the free parameters of the 8-parameter fit are shown in Table 14.

The data support the hypothesis of lepton universality. Overall, the measurements are well reproduced by the Standard Model predictions. At $161 \mathrm{GeV}$, however, the measured total hadronic cross-section is 3.1 statistical standard deviations larger than the theoretical prediction. The difference is not concentrated at high values of $\sqrt{\mathrm{s}^{\prime}} / \sqrt{\mathrm{s}}$ and the cross-section measured at higher collision energy (i.e. $172 \mathrm{GeV}$ ) agrees well with the theory. The difference is therefore likely to originate from a fluctuation of the event rate.

Figures 5 and 6 show the measured hadron, muon and tau cross-sections and forwardbackward asymmetries from the $Z^{0}$-peak energies up to $172 \mathrm{GeV}$. The electron crosssection and forward-backward asymmetry including full $(s+t)$ contribution are presented in Figures 7 and 8. The curves show the ZFITTER, or TOPAZ0 (in the case of electrons), predictions.

\footnotetext{
${ }^{3}$ The correlations $\delta R_{q} / \delta R_{x}, \delta A_{F B}^{q} / \delta A_{F B}^{x}$ and $\delta A_{F B}^{q} / \delta R_{x}$, are given for the working point where the ratios and asymmetries are equal to the SM predictions and are only valid for small deviations from these expectations.
} 


\begin{tabular}{|c|c|c|c|c|c|}
\hline \multicolumn{6}{|c|}{$R_{q}(\sqrt{s}=130-136 \mathrm{GeV})$} \\
\hline quark flavour & $R_{q}$ & $R_{q}^{S M}$ & $\delta R_{q} / \delta R_{b}$ & $\delta R_{q} / \delta R_{c}$ & $\delta R_{q} / \delta R_{u d s}$ \\
\hline bottom & $0.174 \pm 0.028$ & 0.182 & 1 & 0.20 & 0.02 \\
\hline charm & $0.199 \pm 0.073$ & 0.225 & 0.40 & 1 & 0.38 \\
\hline light uds & $0.610 \pm 0.050$ & 0.593 & 0.13 & 0.73 & 1 \\
\hline \multicolumn{6}{|c|}{$R_{q}(\sqrt{s}=161-172 \mathrm{GeV})$} \\
\hline quark flavour & $R_{q}$ & $R_{q}^{S M}$ & $\delta R_{q} / \delta R_{b}$ & $\delta R_{q} / \delta R_{c}$ & $\delta R_{q} / \delta R_{u d s}$ \\
\hline bottom & $0.142 \pm 0.024$ & 0.165 & 1 & 0.17 & 0.02 \\
\hline charm & $0.314 \pm 0.055$ & 0.250 & 0.49 & 1 & 0.43 \\
\hline light uds & $0.581 \pm 0.047$ & 0.585 & 0.14 & 0.64 & 1 \\
\hline
\end{tabular}

\begin{tabular}{|c|c|c|c|c|cc|c|}
\hline \multicolumn{8}{|c|}{$\boldsymbol{A}_{\boldsymbol{F B}}^{q}(\sqrt{\boldsymbol{s}}=\mathbf{1 3 0} \mathbf{1 3 6} \mathbf{G e V})$} \\
\hline quark flavour & $A_{F B}^{q}$ & $A_{F B}^{S M}$ & $\delta A_{F B}^{q} / \delta A_{F B}^{b}$ & $\delta A_{F B}^{q} / \delta A_{F B}^{c}$ & $\delta A_{F B}^{q} / \delta A_{F B}^{s(d)}$ & $\delta A_{F B}^{q} / \delta A_{F B}^{u}$ \\
\hline \hline bottom & $0.67 \pm 0.39$ & 0.475 & 1 & -0.17 & 0.02 & $(0.02)$ & -0.03 \\
\hline charm & $0.90 \pm 1.17$ & 0.679 & -0.48 & 1 & 0.46 & $(0.46)$ & -0.63 \\
\hline strange & $0.95 \pm 0.68$ & 0.473 & 0.12 & -0.60 & 1 & $(0.95)$ & -1.37 \\
\hline up & $0.30 \pm 0.54$ & 0.679 & -0.09 & 0.44 & -0.70 & $(-0.70)$ & 1 \\
\hline down & $0.95 \pm 0.67$ & 0.473 & 0.13 & -0.63 & 1.05 & $(1)$ & -1.45 \\
\hline \hline \multicolumn{7}{|c|}{$A_{F B}^{q}(\sqrt{\boldsymbol{s}}=\mathbf{1 6 1}-\mathbf{1 7 2} \mathbf{G e V})$} \\
\hline quark flavour & $A_{F B}^{q}$ & $A_{F B}^{S M}$ & $\delta A_{F B}^{q} / \delta A_{F B}^{b}$ & $\delta A_{F B}^{q} / \delta A_{F B}^{c}$ & $\delta A_{F B}^{q} / \delta A_{F B}^{s(d)}$ & $\delta A_{F B}^{q} / \delta A_{F B}^{u}$ \\
\hline \hline bottom & $1.05 \pm 0.35$ & 0.545 & 1 & -0.17 & 0.02 & $(0.02)$ & -0.03 \\
\hline charm & $1.14 \pm 0.81$ & 0.663 & -0.48 & 1 & 0.43 & $(0.46)$ & -0.71 \\
\hline strange & $0.48 \pm 0.65$ & 0.543 & 0.13 & -0.65 & 1 & $(0.95)$ & -1.67 \\
\hline up & $0.70 \pm 0.39$ & 0.663 & -0.08 & 0.39 & $-0.59(-0.59)$ & 1 \\
\hline down & $0.48 \pm 0.64$ & 0.543 & 0.14 & -0.69 & 1.06 & $(1)$ & -1.78 \\
\hline
\end{tabular}

\begin{tabular}{|c|c|c|cc|c|}
\hline \multicolumn{6}{|c|}{$\boldsymbol{\delta} \boldsymbol{A}_{\boldsymbol{F B}}^{\boldsymbol{q}} / \boldsymbol{\delta} \boldsymbol{R}_{\boldsymbol{q}}(\sqrt{\boldsymbol{s}}=\mathbf{1 3 0}-\mathbf{1 3 6} \mathbf{G e V})$} \\
\hline quark flavour & $\delta A_{F B}^{q} / \delta R_{b}$ & $\delta A_{F B}^{q} / \delta R_{c}$ & $\delta A_{F B}^{q} / \delta R_{s(d)}$ & $\delta A_{F B}^{q} / \delta R_{u}$ \\
\hline \hline bottom & 1.0 & -0.80 & 0.02 & $(0.02)$ & -0.12 \\
\hline charm & -1.6 & 2.1 & -1.5 & $(-1.5)$ & 1.6 \\
\hline strange & 0.38 & -1.4 & 2.9 & $(2.9)$ & -3.6 \\
\hline up & -0.27 & 1.1 & -2.1 & $(-2.1)$ & 2.6 \\
\hline down & 0.41 & -1.5 & 3.1 & $(3.1)$ & -3.8 \\
\hline \hline \multicolumn{6}{|c|}{$\boldsymbol{\delta} \boldsymbol{A}_{\boldsymbol{F B}}^{q} / \boldsymbol{\delta} \boldsymbol{R}_{\boldsymbol{q}}(\sqrt{\boldsymbol{s}}=\mathbf{1 6 1}-\mathbf{1 7 2} \mathbf{G e V})$} \\
\hline quark flavour & $\delta A_{F B}^{q} / \delta R_{b}$ & $\delta A_{F B}^{q} / \delta R_{c}$ & $\delta A_{F B}^{q} / \delta R_{s(d)}$ & $\delta A_{F B}^{q} / \delta R_{u}$ \\
\hline \hline bottom & 1.2 & -0.77 & 0.03 & $(0.03)$ & -0.12 \\
\hline charm & -1.9 & 1.8 & -1.6 & $(-1.6)$ & 1.6 \\
\hline strange & 0.52 & -1.4 & 3.7 & $(3.7)$ & -3.8 \\
\hline up & -0.31 & 0.8 & -2.2 & $(-2.2)$ & 2.3 \\
\hline down & 0.55 & -1.4 & 3.9 & $(3.9)$ & -4.1 \\
\hline
\end{tabular}

Table 12: Results for flavour tagged samples at energies of 130-136 and 161-172 GeV with $\sqrt{\mathrm{s}^{\prime}} / \sqrt{\mathrm{s}}>0.85$ : the derived quark cross-section ratios, $R_{q}$, and the observed forward-backward quark asymmetry, $A_{F B}^{q}$, together with the Standard Model expectations $R_{q}^{S M}$ and $A_{F B}^{S M}$ from ZFITTER. The correlations between the cross-section ratios, $R_{q}$, and the correlations between the forward-backward asymmetries are given, normalised so that the correlation is 1 for quarks of the same flavour. Also given are the correlations between the forward-backward asymmetries and the values of $R_{q}$. The correlations are calculated at the working point where all values agree with the Standard Model. 


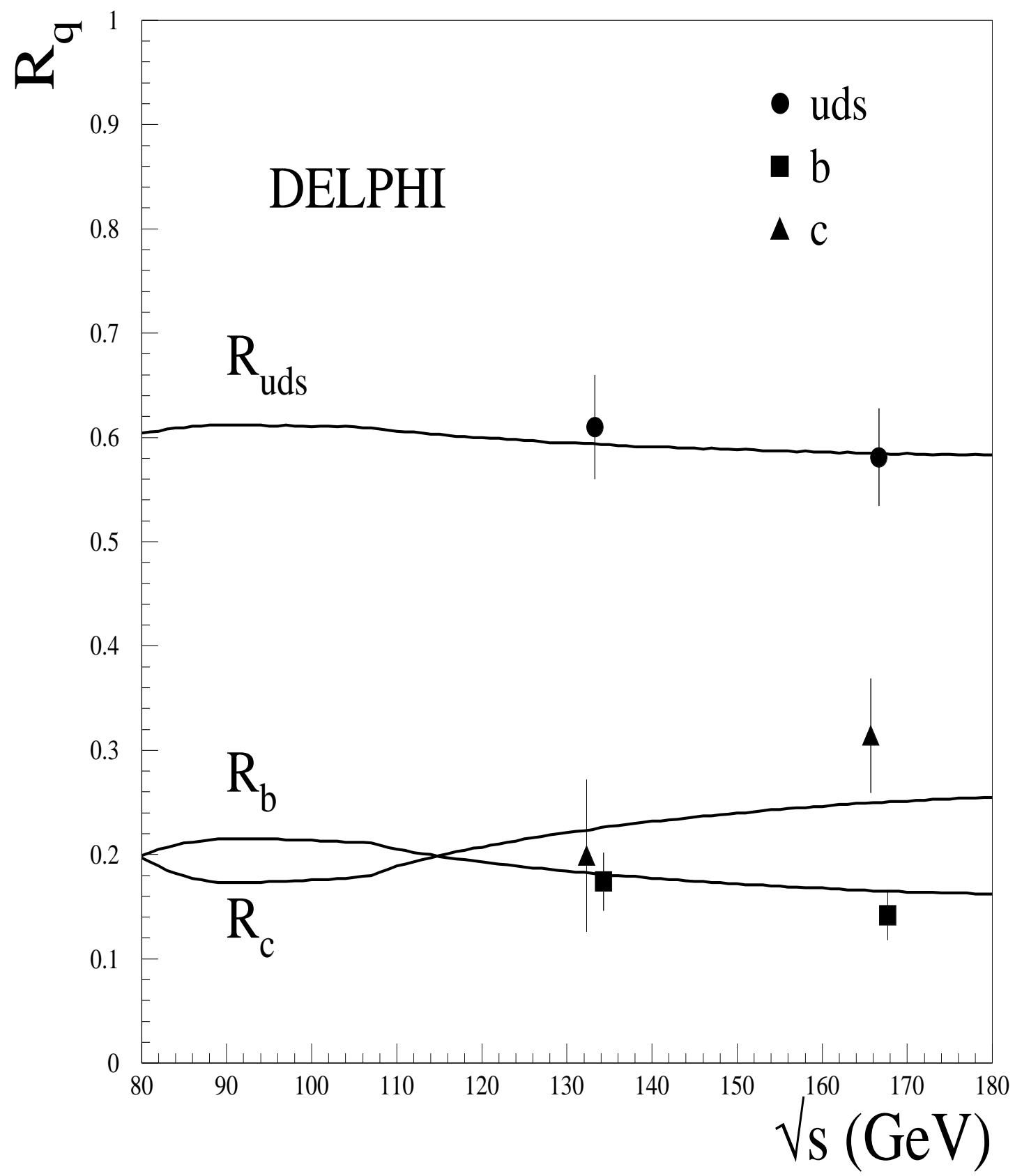

Figure 10: The measured ratios $R_{q}$ of cross-sections for bottom, charm and light uds quarks production compared to the total hadronic cross-section as a function of the centre-of-mass energy, together with the predictions from ZFITTER. Note that in the extraction of the individual cross-section ratios $R_{q}$, the other cross-section ratios are taken from the Standard Model. 


\begin{tabular}{|c|c|c|}
\hline Parameter & Value & SM prediction \\
\hline$M_{\mathrm{Z}}[\mathrm{GeV}]$ & $91.185 \pm 0.006$ & - \\
\hline$\Gamma_{\mathrm{z}}[\mathrm{GeV}]$ & $2.487 \pm 0.004$ & 2.493 \\
\hline $\mathrm{r}_{\text {had }}^{\text {tot }}$ & $2.951 \pm 0.010$ & 2.959 \\
\hline$r_{e}^{\text {tot }}$ & $0.1411 \pm 0.0009$ & \\
\hline $\mathrm{r}_{\mu}^{\mathrm{tot}}$ & $0.1426 \pm 0.0007$ & \\
\hline $\mathrm{r}_{\tau}^{\mathrm{tot}}$ & $0.1418 \pm 0.0010$ & \\
\hline$r_{\ell}^{\text {tot }}$ & $0.1421 \pm 0.0006$ & 0.1425 \\
\hline $\mathrm{j}_{\text {had }}^{\text {tot }}$ & $0.32 \pm 0.29$ & 0.22 \\
\hline$j_{\mathrm{e}}^{\text {tot }}$ & $-0.039 \pm 0.046$ & \\
\hline $\mathrm{j}_{\mu}^{\text {tot }}$ & $0.050 \pm 0.030$ & \\
\hline $\mathrm{j}_{\tau}^{\text {tot }}$ & $0.014 \pm 0.037$ & \\
\hline$j_{\ell}^{\text {tot }}$ & $0.022 \pm 0.023$ & 0.004 \\
\hline $\mathrm{r}_{\mathrm{e}}^{\mathrm{fb}}$ & $0.0033 \pm 0.0009$ & \\
\hline $\mathrm{r}_{\mu}^{\mathrm{fb}}$ & $0.0028 \pm 0.0005$ & \\
\hline $\mathrm{r}_{\tau}^{\mathrm{fb}}$ & $0.0042 \pm 0.0007$ & \\
\hline $\mathrm{r}_{\ell}^{\mathrm{fb}}$ & $0.00324 \pm 0.00038$ & 0.00265 \\
\hline $\mathrm{j}_{\mathrm{e}}^{\mathrm{fb}}$ & $0.82 \pm 0.07$ & \\
\hline $\mathrm{j}_{\mu}^{\mathrm{fb}}$ & $0.763 \pm 0.034$ & \\
\hline $\mathrm{j}_{\tau}^{\mathrm{fb}}$ & $0.746 \pm 0.044$ & \\
\hline$\dot{j}_{\ell}^{\mathrm{fb}}$ & $0.763 \pm 0.025$ & 0.799 \\
\hline
\end{tabular}

Table 13: Results of the 16- and 8-parameter fits to the combined line-shape and high energy data. Also shown are the Standard Model predictions for the fit parameters evaluated for $M_{\mathrm{Z}}$ as given in the table, $M_{H}=300 \mathrm{GeV} / c^{2}, m_{t}=175 \mathrm{GeV} / c^{2}$ and $\alpha_{s}\left(M_{\mathrm{Z}}\right)=0.118$ 
The correlation between the parameters $M_{\mathrm{Z}}$ and $\mathrm{j}_{\text {had }}^{\text {tot }}$ is shown in Figure 11 . It can be seen that a significant improvement in the precision on the hadronic interference parameter, $\mathrm{j}_{\text {had }}^{\text {tot }}$, is obtained when the high energy data are included in the fits.

\section{Physics Beyond the Standard Model}

Data from $e^{+} e^{-}$collisions at LEP-2 energies can be used to put severe constraints on physics beyond the Standard Model. Such measurements could have an impact on the interpretation of measurements from other experiments, for example [5-7].

Interactions not described by the Standard Model can influence the differential crosssections for the fermion pair production, $e^{+} e^{-} \rightarrow f \bar{f}$, leading to deviations from the Standard Model predictions. For example, the existence of a new particle with a mass of around $200 \mathrm{GeV} / \mathrm{c}^{2}$, would produce virtual effects at lower energies, such that the crosssections and asymmetries for different quark flavours and lepton species at LEP2 would be different from the SM predictions. The values of the cross-sections and forward-backward asymmetries for fermion pair production measured by the DELPHI collaboration at $\sqrt{s}=$ $130-172 \mathrm{GeV}$ were used to search for such effects in the models discussed below. The results of this interpretation of the data are given in section 6 .

\subsection{Contact interactions}

The first set of models considered here involve contact interactions between the initial and final state fermionic currents. Such models provide a general description of the low energy behaviour of new physics with a high characteristic energy scale. Following reference [29] these interactions are parameterised by an effective Lagrangian, added to the Standard Model Lagrangian $\mathcal{L}_{\text {eff }}$, of the form:

$$
\mathcal{L}_{e f f}=\frac{g^{2}}{(1+\delta) \Lambda^{2}} \sum_{i, j=L, R} \eta_{i j} \bar{e}_{i} \gamma_{\mu} e_{i} \bar{f}_{j} \gamma^{\mu} f_{j},
$$

where $g^{2} / 4 \pi$ is taken to be 1 by convention, $\delta=1(0)$ for $f=e(f \neq e), \eta_{i j}= \pm 1$ or 0 , $\Lambda$ is the scale of the contact interactions ${ }^{4}, e_{i}$ and $f_{j}$ are are left or right-handed spinors. By assuming different helicity coupling between the initial state and final state currents and either constructive or destructive interference with the Standard Model (according to the choice of each $\eta_{i j}$ ) a basic set of 12 different models can be defined from this Lagrangian [30]. The differential cross-section for scattering the outgoing fermion at an angle $\theta$ with respect to the incident $e^{-}$direction is given by $[31,32]$

$$
\frac{d \sigma}{d \cos \theta}=\frac{\pi \alpha^{2}}{2 s} N_{c}^{f}\left\{\begin{array}{c}
{\left[\left|\bar{A}_{L R}^{e e}\right|^{2}+\left|\bar{A}_{R L}^{e e}\right|^{2}\right]\left(\frac{s}{t}\right)^{2} \delta+} \\
{\left[\left|A_{L R}^{e f}\right|^{2}+\left|A_{R L}^{e f}\right|^{2}\right]\left(\frac{t}{s}\right)^{2}+} \\
{\left[\left|A_{L L}^{e f}\right|^{2}+\left|A_{R R}^{e f}\right|^{2}\right]\left(\frac{u}{s}\right)^{2}}
\end{array}\right\},
$$

where $s, t$ and $u$ are the Mandelstam variables and $N_{c}^{f}$ is the number of colours for fermion $f$. The $A_{i j}$ and $\bar{A}_{i j}$ are helicity amplitudes for the scattering process. When the helicity amplitudes are squared, 3 sets of terms arise: the first set contains purely Standard Model terms; the second set of terms derive from the interference between

\footnotetext{
${ }^{4}$ The choice of $g^{2}$ is somewhat arbitrary; if the coupling constant was taken to be $\alpha_{s}$ much lower limits on $\Lambda$ would be obtained.
} 


\section{DELPHI}

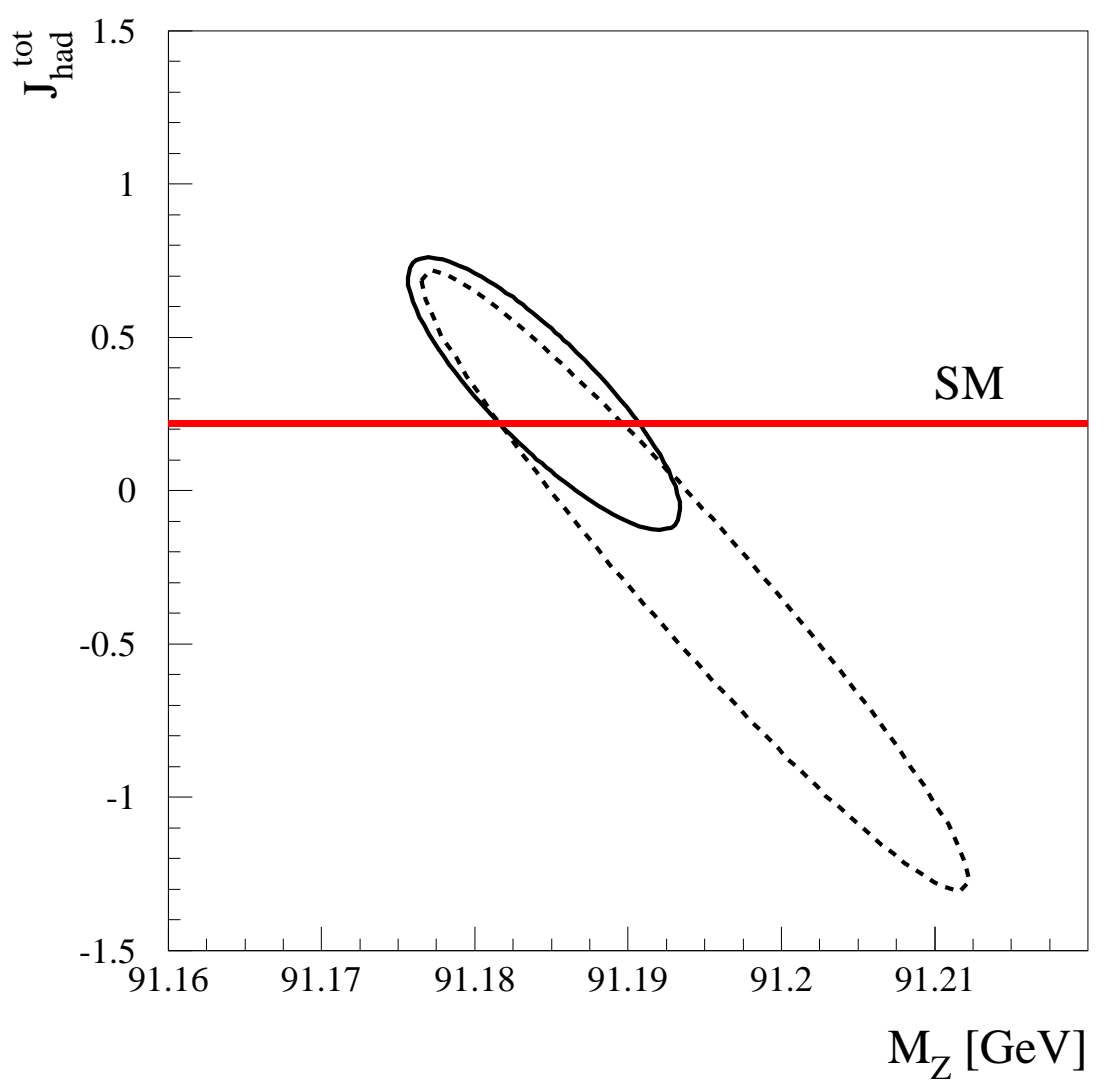

Figure 11: Probability contour plot in the $M_{\mathrm{Z}} \mathrm{j}_{\text {had }}^{\text {tot }}$ plane. The dotted curve shows the region accepted at the $68 \%$ confidence level from a fit to data taken at the energies around $Z^{0}$; the solid curve show the region accepted at the same confidence level when the high energy data are also included in the fit.

\begin{tabular}{|c|c|c|c|c|c|c|c|}
\hline & $\Gamma_{Z}$ & $\mathrm{r}_{\text {had }}^{\text {tot }}$ & $\mathrm{r}_{\ell}^{\mathrm{tot}}$ & $\mathrm{j}_{\mathrm{had}}^{\text {tot }}$ & $j_{\ell}^{\text {tot }}$ & $\mathrm{r}_{\ell}^{\mathrm{fb}}$ & $\mathrm{j}_{\ell}^{\mathrm{fb}}$ \\
\hline$M_{\mathrm{Z}}$ & -.15 & -.11 & -.09 & -.85 & -.54 & .17 & -.04 \\
\hline$\Gamma_{\mathrm{Z}}$ & & .84 & .69 & .21 & .12 & .00 & .08 \\
\hline $\mathrm{r}_{\text {had }}^{\text {tot }}$ & & & .73 & .17 & .09 & .01 & .08 \\
\hline $\mathrm{r}_{\ell}^{\text {tot }}$ & & & & .13 & .14 & .03 & .12 \\
\hline $\mathrm{j}_{\text {had }}^{\text {tot }}$ & & & & & .52 & -.15 & .04 \\
\hline $\mathrm{j}_{\ell}^{\text {tot }}$ & & & & & & -.06 & .03 \\
\hline $\mathrm{r}_{\ell}^{\mathrm{fb}}$ & & & & & & & .15 \\
\hline
\end{tabular}

Table 14: Correlation matrix of the 8-parameter fit. 


\begin{tabular}{|c|c|c|l|}
\hline$i j k$ & limit & C.L. (\%) & Derived from \\
\hline \hline 121 & 0.10 & 95 & Charged Current Universality \\
122 & 0.10 & 95 & Charged Current Universality \\
123 & 0.10 & 95 & Charged Current Universality \\
\hline 131 & 0.16 & 95 & Tau Decay \\
132 & 0.16 & 95 & Tau Decay \\
\hline 133 & 0.006 & - & Mass of $\nu_{e}$ limit $<5 \mathrm{eV} / \mathrm{c}^{2}$ \\
\hline 231 & 0.16 & 95 & Tau Decay \\
232 & 0.16 & 95 & Tau Decay \\
233 & 0.16 & 95 & Tau Decay \\
\hline
\end{tabular}

Table 15: Existing upper limits on $\lambda_{i j k}$ for assumed sneutrino masses of $200 \mathrm{GeV} / c^{2}$.

contact interactions and the Standard Model, these terms are proportional to $1 / \Lambda^{2}$; the final set of terms are due to contact interactions alone and are proportional to $1 / \Lambda^{4}$. For the purpose of fitting contact interaction models to the data, a new parameter $\epsilon=1 / \Lambda^{2}$ is defined; with $\epsilon=0$ in the limit that there are no contact interactions. This parameter is allowed to take both positive and negative values in the fits. It is worth noting that there is a symmetry between models with $\eta_{i j}=+1$ and those with $\eta_{i j}=-1$. The predicted differential cross-section in the constructive $(+)$ models is the same as the destructive $(-)$ models for $\epsilon^{-}=-\epsilon^{+}$.

\subsection{Sneutrino exchange models}

The second set of models consider possible $s$ or $t$ channel sneutrino $\tilde{\nu}_{\ell}$ exchange in R-parity violating supersymmetry [33], which can affect the channel $e^{+} e^{-} \rightarrow l^{+} l^{-}$. The purely leptonic part of the R-parity violating superpotential has the form

$$
\lambda_{i j k} L_{L}^{i} L_{L}^{j} \bar{E}_{R}^{k}
$$

where $i j k$ are generation indices, $L_{L}$ represents a left-handed leptonic superfield doublet and $\bar{E}_{R}$ corresponds to the right-handed singlet superfield of charged leptons. The coupling $\lambda_{i j k}$ is only non-zero for combinations involving at least two generations and for $i<j$.

For the channel $e^{+} e^{-} \rightarrow e^{+} e^{-}$there are possible contributions from the $s$-channel production and $t$-channel exchange of either $\tilde{\nu_{\mu}}\left(\lambda_{121} \neq 0\right)$ or $\tilde{\nu_{\tau}}\left(\lambda_{131} \neq 0\right)$. For the channels $e^{+} e^{-} \rightarrow \mu^{+} \mu^{-}$and $e^{+} e^{-} \rightarrow \tau^{+} \tau^{-}$there is no $s$-channel contribution if only one of the $\lambda_{i j k}$ is non-zero. For $e^{+} e^{-} \rightarrow \mu^{+} \mu^{-}$there are $t$-channel contributions from either $\tilde{\nu_{e}}\left(\lambda_{121} \neq 0\right), \tilde{\nu_{\mu}}\left(\lambda_{122} \neq 0\right)$ or from $\tilde{\nu_{\tau}}\left(\lambda_{132}\right.$ or $\left.\lambda_{231} \neq 0\right)$. If both $\lambda_{131} \neq 0$ and $\lambda_{232} \neq 0$ then the $s$-channel production of $\tilde{\nu}_{\tau}$ is possible. For $e^{+} e^{-} \rightarrow \tau^{+} \tau^{-}$there are $t$-channel contributions from either $\tilde{\nu_{e}}\left(\lambda_{131} \neq 0\right), \tilde{\nu_{\mu}}\left(\lambda_{123}\right.$ or $\left.\lambda_{232} \neq 0\right)$ or from $\tilde{\nu_{\tau}}\left(\lambda_{133} \neq 0\right)$. If both $\lambda_{121} \neq 0$ and $\lambda_{233} \neq 0$ then there the $s$-channel production of $\tilde{\nu_{\mu}}$ is possible.

In this paper all these possibilities are considered. For a given scenario the $s$ or t-channel sneutrino exchange amplitude contribution is added to the Standard Model contribution as appropriate. If there is no sneutrino exchange for a specific channel then the prediction for that channel is just the SM value.

In the case of $s$-channel sneutrino graphs, if the sneutrino mass, $m_{\tau}$, is equal, or close, to the centre-of-mass energy of the $e^{+} e^{-}$beams, resonant sneutrino production occurs, 


\begin{tabular}{|c|c|c|l|}
\hline$i j k$ & limit & C.L. (\%) & Derived from \\
\hline \hline 111 & 0.001 & 68 & Neutrinoless double beta decay \\
\hline 112 & 0.028 & 95 & Charged current universality \\
113 & 0.028 & 95 & Charged current universality \\
\hline 121 & 0.034 & 95 & Atomic parity violation \\
131 & 0.034 & 95 & Atomic parity violation \\
\hline 122 & 0.06 & - & Mass of $\nu_{e}$ limit $<5 \mathrm{eV} / \mathrm{c}^{2}$ \\
133 & 0.002 & - & Mass of $\nu_{e}$ limit $<5 \mathrm{eV} / \mathrm{c}^{2}$ \\
\hline 123 & 0.30 & 95 & $D^{0}-\overline{D^{0}}$ mixing \\
132 & 0.48 & 95 & $\Gamma($ Hadron $) / \Gamma($ Lepton $)$ of $\mathrm{Z}$ \\
\hline
\end{tabular}

Table 16: Existing upper limit on $\lambda_{i j k}^{\prime}$ for assumed squark masses of $200 \mathrm{GeV} / c^{2}$.

which can lead to a large change in the cross-section. A lesser change in the cross-section will occur for $m_{\tilde{\nu}}<\sqrt{s}$ due to the process of radiative return. There is some sensitivity to $m_{\sim}$ just above $\sqrt{s}$ due to the finite width of the particle. It is assumed here that the sneutrino width is $1 \mathrm{GeV}$.

Existing upper limits on $\lambda_{i j k}$ are summarised in table 15; for further details see [34] and references therein.

\subsection{Squark exchange}

In supersymmetric extensions to the Standard Model which include R-parity violation, as well as a purely leptonic term in the superpotential there is a further term which links the lepton and quark sectors:

$$
\lambda_{i j k}^{\prime} L_{L}^{i} Q_{L}^{j} \bar{D}_{R}^{k}
$$

where $L_{L}$ and $Q_{L}$ represent left-handed superfield doublets of leptons and quarks and $\bar{D}_{R}$ corresponds to the right-handed singlet superfield of down type quarks, as above ijk are generation indices.

This term in the superpotential could also generate R-parity violating SUSY terms in the channel $e^{+} e^{-} \rightarrow q \bar{q}$. At LEP $e^{+} e^{-}$pairs might annihilate through the $t$-channel exchange of a squark of mass $m$ and produce a quark-antiquark pair in the final state. If only one of the $\lambda_{i j k}^{\prime}$ is non zero, and only one squark is light, then the production of only one quark species will be affected, for example if $\lambda_{121}^{\prime} \neq 0$ and the $\tilde{d}$ is light then charm production will be affected. The same term, with suitable $\lambda_{i j k}^{\prime} \neq 0$, could give rise to the following interactions: (i) $e^{+} d \rightarrow \tilde{u}_{L}$, (ii) $e^{+} s \rightarrow \tilde{u}_{L}$ (iii) $e^{+} \bar{u} \rightarrow \tilde{d}_{R}$, which have been suggested as possible explanations for the anomaly presented in [5].

In the analysis presented here, each $q \bar{q}$ final state was considered separately assuming that the production of all other channels was fixed by the Standard Model. The predictions used in this paper were based on the formalism of [6], which makes use of the property that squark exchange is equivalent to certain models of leptoquark exchange ${ }^{5}$. The coupling constant is denoted by $\lambda_{\text {susy }}^{\prime}$ for each channel, this can be explicitly converted to the appropriate $\lambda_{i j k}^{\prime}$ as desired. For final states with down type quarks, the

\footnotetext{
${ }^{5}$ The limits presented here can therefore be treated as limits on leptoquark exchange for models of the form $\tilde{S}_{1 / 2}$ or $S_{0}$ with coupling constant $g=\lambda_{s u s y}^{\prime}$ see equation 5 and table 2 of [6]. Note also that in the large mass limit the effective Lagrangian corresponds to a contact interaction of the form $L R$ with $\eta=-1 / 2$ for up type quarks or $L L$ with $\eta=+1 / 2$ for down type quarks.
} 
cross-section increases as a function of $\lambda_{\text {susy }}^{\prime}$; for up type quarks, the cross-section first decreases and then increases due to the interference, this is shown later in Figure 14, where a squark mass of $200 \mathrm{GeV} / c^{2}$ is assumed for $\sqrt{s}=166 \mathrm{GeV}$. Existing upper limits on $\lambda_{i j k}^{\prime}$ are given in table 16, for further details see [34] and references therein.

\subsection{Fermion production in presence of a $\mathrm{Z}^{\prime}$-boson}

Many theories which are more general than the Standard Model predict the existence of additional heavy gauge bosons. The consequences of several of these models were investigated, complemented by a model independent fit to the leptonic data.

\subsubsection{Specific $Z^{\prime}$ Models}

The existence of an additional heavy gauge boson $Z^{\prime}$ can be parameterised by the mass of the boson $M_{Z^{\prime}}$ and by its couplings to fermions. In addition, a possible mixing between the $Z^{\prime}$ and the standard $Z$, represented by a mixing angle $\theta_{Z Z^{\prime}}$, has to be taken into account $[35,36]$. In order to deal with a restricted number of free parameters, it is useful to consider specific $Z^{\prime}$-models with well defined couplings. Popular models are:

- The $E_{6}$ model [37]. It is based on a symmetry breaking of the $E_{6}$ GUT. The free parameter of this model is the mixing of the $Z^{\prime}$ to fermions, $\Theta_{6}$. Usual choices of $\Theta_{6}$ are $\Theta_{6}=0$ ( $\chi$-model $), \pi / 2(\psi$-model $)$ and $\Theta_{6}=-\arctan \sqrt{5 / 3}(\eta$-model $)$.

- The L-R model [38]. It includes a right-handed $S U(2)_{R}$ extension to the Standard Model gauge group $S U(2)_{L} \otimes U(1)$. The free parameter $\alpha_{L R}$ describes the coupling of the heavy bosons to fermions. $\alpha_{L R}$ varies between $\sqrt{2 / 3} \leq \alpha_{L R} \leq \sqrt{\cot ^{2} \theta_{W}-1}$.

\subsubsection{Model independent approach}

In a more general approach, the $\mathrm{Z}^{\prime}$-boson is directly described in terms of its couplings $a_{f}^{\prime}$ and $v_{f}^{\prime}$. The amplitude for fermion pair production in $e^{+} e^{-}$annihilations via a $Z^{\prime}$ exchange has the following expression at the Born level [39]:

$$
\begin{aligned}
\mathcal{M}\left(\mathrm{Z}^{\prime}\right) & =\frac{g_{2}^{2}}{s-m_{\mathrm{Z}^{\prime}}^{2}} \bar{u}_{e} \gamma_{\beta}\left(\gamma_{5} a_{e}^{\prime}+v_{e}^{\prime}\right) u_{e} \bar{u}_{f} \gamma^{\beta}\left(\gamma_{5} a_{f}^{\prime}+v_{f}^{\prime}\right) u_{f} \\
& =-\frac{4 \pi}{s} \bar{u}_{e} \gamma_{\beta}\left(\gamma_{5} a_{e}^{N}+v_{e}^{N}\right) u_{e} \bar{u}_{f} \gamma^{\beta}\left(\gamma_{5} a_{f}^{N}+v_{f}^{N}\right) u_{f}
\end{aligned}
$$

with

$$
a_{f}^{N}=a_{f}^{\prime} \sqrt{\frac{g_{2}^{2}}{4 \pi} \frac{s}{m_{\mathrm{Z}^{\prime}}^{2}-s}}, \quad v_{f}^{N}=v_{f}^{\prime} \sqrt{\frac{g_{2}^{2}}{4 \pi} \frac{s}{m_{\mathrm{Z}^{\prime}}^{2}-s}}, \quad m_{\mathrm{Z}^{\prime}}^{2}=M_{\mathrm{Z}^{\prime}}^{2}-i \Gamma_{\mathrm{Z}^{\prime}} M_{\mathrm{Z}^{\prime}}
$$

Off the $Z^{\prime}$ resonance, pair production is only sensitive to the normalised couplings $a_{f}^{N}$ and $v_{f}^{N}$. As a consequence, the couplings and the mass of the $\mathrm{Z}^{\prime}$ boson cannot be measured independently. Furthermore, the coupling constant $g_{2}$ is unknown outside of any specific model. In the following the convention is taken that $\frac{g_{2}^{2}}{4 \pi}=1$. The normalised couplings then become

$$
a_{f}^{N}=a_{f}^{\prime} \sqrt{\frac{s}{m_{\mathrm{Z}^{\prime}}^{2}-s}}, \quad v_{f}^{N}=v_{f}^{\prime} \sqrt{\frac{s}{m_{\mathrm{Z}^{\prime}}^{2}-s}} .
$$




\section{$6 \quad$ Fits to physics beyond the Standard Model}

\subsection{Leptonic channels}

The input to the fits consisted of the cross-sections for scattering of the negatively charged lepton into the forward and backward hemispheres, defined with respect to the incident electron direction (as seen in the laboratory frame) for the non-radiative class of $e^{+} e^{-}, \mu^{+} \mu^{-}$and $\tau^{+} \tau^{-}$final states, at $\sqrt{s}=130-172 \mathrm{GeV}$.

Theoretical predictions were based on the appropriate Born level expressions convoluted with QED corrections, the Standard Model contributions being treated within the Improved Born approximation [40], with the following parameters:

$$
\begin{array}{rll}
m_{t}=175 \pm 6 \mathrm{GeV} / c^{2} & , & M_{H}=300_{-240}^{+700} \mathrm{GeV} / c^{2} \\
\alpha_{s}=0.118 \pm 0.005 & , & M_{\mathrm{Z}}=91.187 \pm 0.007 \mathrm{GeV} / c^{2}
\end{array}
$$

Which were evaluated from data at the $\mathrm{Z}$ resonance, using hadronic final states only. The ranges given are the values over which the parameters were varied to estimate the bias from uncertainties in the Standard Model inputs. The changes in the fits, presented below, were found to be negligible. The systematic errors from knowledge of the Standard Model parameters were therefore neglected. For the $\mu^{+} \mu^{-}$and $\tau^{+} \tau^{-}$final states the Standard Model contributions were computed using ZFITTER, with full QED radiative corrections, including the effects of box diagrams and initial-final state interference. The treatment of interference between initial state and final state radiation was a potential source of uncertainty in the radiative corrections to the Standard Model and the new physics as this correction was calculated at lower order than other QED corrections [41]. Including or excluding the interference led to changes in the corrected forward and backward crosssections of approximately $2.5 \%$. The resulting uncertainties in the fitted values were negligible when added in quadrature with the statistical errors. For the $e^{+} e^{-}$final state the SM computations were made using TOPAZ0.

QED radiative corrections for the new physics contributions to the cross-sections, were calculated using the MIBA package [42]. These corrections were checked against ZFITTER, DYMU3 and TOPAZ0. The radiative corrections for new physics differ from the corrections for the Standard Model. This difference arises from initial state radiation. The probability of radiating photons to arrive at a given centre-of-mass energy depends on the energy dependence of the Born level cross-section. Models which contain new physics have a different $s$ dependence to the Standard Model. For the range of new physics parameters extracted in this paper the difference in radiative corrections are rather negligible (less than 1\%) compared to the sizeable statistical errors, but were nevertheless taken into account in the fit.

\subsubsection{Contact interaction models}

The data were compared to each of the 12 Contact Interaction models mentioned above ${ }^{6}$ considering separately the $e^{+} e^{-}, \mu^{+} \mu^{-}$and $\tau^{+} \tau^{-}$final states, and all three final states combined, assuming lepton universality in the contact interactions.

The values of $\epsilon$ extracted for each model were all compatible with the Standard Model expectation $\epsilon=0$, at the two standard deviation level. The fitted values of $\epsilon$ were converted into $95 \%$ confidence level lower limits on $\Lambda$, and are shown in Table 17 . In the

\footnotetext{
${ }^{6}$ For leptonic final states, models with only $\eta_{L R}= \pm 1$ are equivalent to models with only $\eta_{R L}= \pm 1$.
} 
cases where $\epsilon$ was unphysical, $\epsilon<0$, the following definition was used, $\Lambda=1 / \sqrt{1.64 \sigma}$, where $\sigma$ was the 1 standard deviation parabolic statistical error on $\epsilon$. Otherwise the value was taken to be, $\Lambda^{( \pm)}=1 / \sqrt{ \pm \epsilon^{(+)}+1.64 \sigma_{+}^{(+)}}$where $\sigma_{+}$is the upper 1 standard deviation statistical error on $\epsilon$, and the \pm refer to models with $\eta= \pm 1^{7}$. Figure 12 shows the expected variation with $\sqrt{s}$ of the total cross-section in the $\mathrm{LL}^{ \pm}$models for an energy scale $\Lambda=3 \mathrm{TeV}$ compared to the data for $e^{+} e^{-} \rightarrow \mu^{+} \mu^{-}$and $e^{+} e^{-} \rightarrow \tau^{+} \tau^{-}$as an indication of the constraints the data put on contact interaction models.

\subsubsection{Sneutrino exchange}

The total cross-section and forward-backward asymmetry values for the channels $e^{+} e^{-} \rightarrow e^{+} e^{-}, e^{+} e^{-} \rightarrow \mu^{+} \mu^{-}$and $e^{+} e^{-} \rightarrow \tau^{+} \tau^{-}$, at each centre-of-mass energy, were again used in the fits. The theoretical prediction consisted of Improved Born Approximation Standard Model terms, plus sneutrino exchange, plus interference terms.

All the fits considered result in values of $\lambda$ which are compatible with zero; so results are expressed as $95 \%$ confidence limits. The first fits considered are to those terms which modify the $e^{+} e^{-} \rightarrow e^{+} e^{-}$channel. These involve the $s$ and $t$-channel exchange of a $\tilde{\nu}_{\mu}$ $\left(\lambda_{121} \neq 0\right)$ or $\tilde{\nu_{\tau}}\left(\lambda_{131} \neq 0\right)$. The resulting $95 \%$ limits on $\lambda$, as a function of $m_{\tilde{\nu}}$, are given in Figure 13a. It can be seen that the best limits on $\lambda$ are obtained for the case where $m_{\tilde{\nu}}$ is close to the actual centre-of-mass energy of the LEP collisions, but that the radiative return process gives some sensitivity between these points. It can be seen that $\lambda$ greater than approximately 0.08 can be excluded for $m_{\tilde{\nu}}$ in the present LEP 2 range of energies at the $95 \%$ confidence level.

For the case that only one $\lambda$ value is non-zero there are only $t$-channel sneutrino effects for $e^{+} e^{-} \rightarrow \mu^{+} \mu^{-}$and $e^{+} e^{-} \rightarrow \tau^{+} \tau^{-}$. The values of $\lambda$ obtained for the $e^{+} e^{-} \rightarrow \mu^{+} \mu^{-}$channel and for the $e^{+} e^{-} \rightarrow \tau^{+} \tau^{-}$channel are all consistent with zero, so results are expressed as $95 \%$ confidence exclusion limits in Table 18 .

For the fits assuming that $\lambda_{131}=\lambda_{232}=\lambda$, the resulting $95 \%$ limits on $\lambda$, as a function of $m_{\tilde{\nu}}$, are given in Figure 13b. A similar exclusion pattern to that obtained from the $e^{+} e^{-} \rightarrow e^{+} e^{-}$channel is obtained, $\lambda$ greater than approximately 0.1 can be excluded for $m_{\tilde{\nu}}$ in the present LEP 2 range of energies at the $95 \%$ confidence level. The exclusion contour for $\lambda_{121}=\lambda_{233}=\lambda$ is shown in figure $13 \mathrm{c}$, from which it can be seen that again a similar exclusion pattern is obtained.

The sneutrino width is not constrained within R-parity violating supersymmetry. A value of $1 \mathrm{GeV}$ has been used [6]. If a value of $2 \mathrm{GeV}$ were used then the sensitivity above $172 \mathrm{GeV}$ would be marginally improved.

\subsection{Flavour-tagged events}

Radiative corrections for hadronic final states were considered in a manner similar to the leptonic final states and were found to be negligible to the accuracy of the analysis presented here.

\subsubsection{Squark exchange}

Limits on the coupling constant $\lambda_{\text {susy }}^{\prime}$ were obtained from the data by comparing the measured cross-section ratios with the expected cross-section ratios as a function of $\lambda_{s u s y}^{\prime}$. For this purpose the measured quark cross-section ratios $\left(\sigma_{q}-\sigma^{S M}\right) / \sigma^{S M}$ are extracted,

\footnotetext{
${ }^{7}$ The fitted values of $\epsilon$ for models with $\eta=-1$ are the negative of those with $\eta=+1$
} 


\begin{tabular}{|c|r|c|c|}
\hline \multicolumn{4}{|c|}{$e^{+} e^{-} \rightarrow e^{+} e^{-}$} \\
\hline Model & $\epsilon_{-\sigma_{-}}^{+\sigma+}\left(\mathrm{TeV}^{-2}\right)$ & $\Lambda^{+}(\mathrm{TeV})$ & $\Lambda^{-}(\mathrm{TeV})$ \\
\hline \hline LL & $0.076_{-0.067}^{+0.084}$ & 2.2 & 2.8 \\
\hline RR & $0.080_{-0.070}^{+0.083}$ & 2.2 & 2.8 \\
\hline VV & $0.020_{-0.014}^{+0.014}$ & 4.8 & 6.6 \\
\hline AA & $-0.042_{-0.062}^{+0.040}$ & 3.5 & 2.6 \\
\hline RL & $0.078_{-0.054}^{+0.065}$ & 2.3 & 3.2 \\
\hline LR & $0.078_{-0.054}^{+0.065}$ & 2.3 & 3.2 \\
\hline
\end{tabular}

\begin{tabular}{|c|c|c|c|}
\hline \multicolumn{4}{|c|}{$e^{+} e^{-} \rightarrow \boldsymbol{\mu}^{+} \boldsymbol{\mu}^{-}$} \\
\hline Model & $\epsilon_{-\sigma_{-}}^{+\sigma_{+}}\left(\mathrm{TeV}^{-2}\right)$ & $\Lambda^{+}(\mathrm{TeV})$ & $\Lambda^{-}(\mathrm{TeV})$ \\
\hline \hline LL & $-0.050_{-0.055}^{+0.051}$ & 3.4 & 2.7 \\
\hline RR & $-0.056_{-0.064}^{+0.056}$ & 3.2 & 2.5 \\
\hline VV & $-0.029_{-0.019}^{+0.022}$ & 5.5 & 4.1 \\
\hline AA & $-0.001_{-0.025}^{+0.021}$ & 5.1 & 4.9 \\
\hline RL & $-0.132_{-0.116}^{+0.109}$ & 2.3 & 1.8 \\
\hline LR & $-0.132_{-0.116}^{+0.109}$ & 2.3 & 1.8 \\
\hline
\end{tabular}

\begin{tabular}{|c|r|c|c|}
\hline \multicolumn{4}{|c|}{$e^{+} e^{-} \rightarrow \boldsymbol{\tau}^{+} \boldsymbol{\tau}^{-}$} \\
\hline Model & $\epsilon_{-\sigma_{-}}^{+\sigma_{+}}\left(\mathrm{TeV}^{-2}\right)$ & $\Lambda^{+}(\mathrm{TeV})$ & $\Lambda^{-}(\mathrm{TeV})$ \\
\hline \hline LL & $-0.016_{-0.082}^{+0.069}$ & 2.8 & 2.6 \\
\hline RR & $-0.018_{-0.094}^{+0.076}$ & 2.7 & 2.4 \\
\hline VV & $-0.023_{-0.029}^{+0.029}$ & 4.6 & 3.8 \\
\hline AA & $0.019_{-0.034}^{+0.035}$ & 3.6 & 4.2 \\
\hline RL & $-0.157_{-0.151}^{+0.141}$ & 2.0 & 1.6 \\
\hline LR & $-0.157_{-0.151}^{+0.141}$ & 2.0 & 1.6 \\
\hline
\end{tabular}

\begin{tabular}{|c|r|c|c|}
\hline \multicolumn{4}{|c|}{$\boldsymbol{e}^{+} \boldsymbol{e}^{-} \rightarrow \boldsymbol{l}^{+} \boldsymbol{l}^{-}$} \\
\hline Model & $\epsilon_{-\sigma_{-}}^{+\sigma+}\left(\mathrm{TeV}^{-2}\right)$ & $\Lambda^{+}(\mathrm{TeV})$ & $\Lambda^{-}(\mathrm{TeV})$ \\
\hline LL & $-0.007_{-0.031}^{+0.032}$ & 4.4 & 4.2 \\
\hline RR & $-0.004_{-0.034}^{+0.034}$ & 4.2 & 4.1 \\
\hline VV & $0.000_{-0.009}^{+0.010}$ & 7.7 & 7.9 \\
\hline AA & $-0.006_{-0.016}^{+0.017}$ & 6.1 & 5.5 \\
\hline RL & $0.007_{-0.032}^{+0.030}$ & 4.2 & 4.4 \\
\hline LR & $0.007_{-0.032}^{+0.030}$ & 4.2 & 4.4 \\
\hline
\end{tabular}

Table 17: Fitted values of $\epsilon$ and $95 \%$ confidence limits on the scale, $\Lambda$, of contact interactions in the models discussed in the text, for $e^{+} e^{-} \rightarrow e^{+} e^{-}, e^{+} e^{-} \rightarrow \mu^{+} \mu^{-}, e^{+} e^{-} \rightarrow \tau^{+} \tau^{-}$and $e^{+} e^{-} \rightarrow l^{+} l^{-}$, a combination of the above assuming lepton universality in the contact interactions. The errors on $\epsilon$ are statistical only. 


\section{DELPHI}

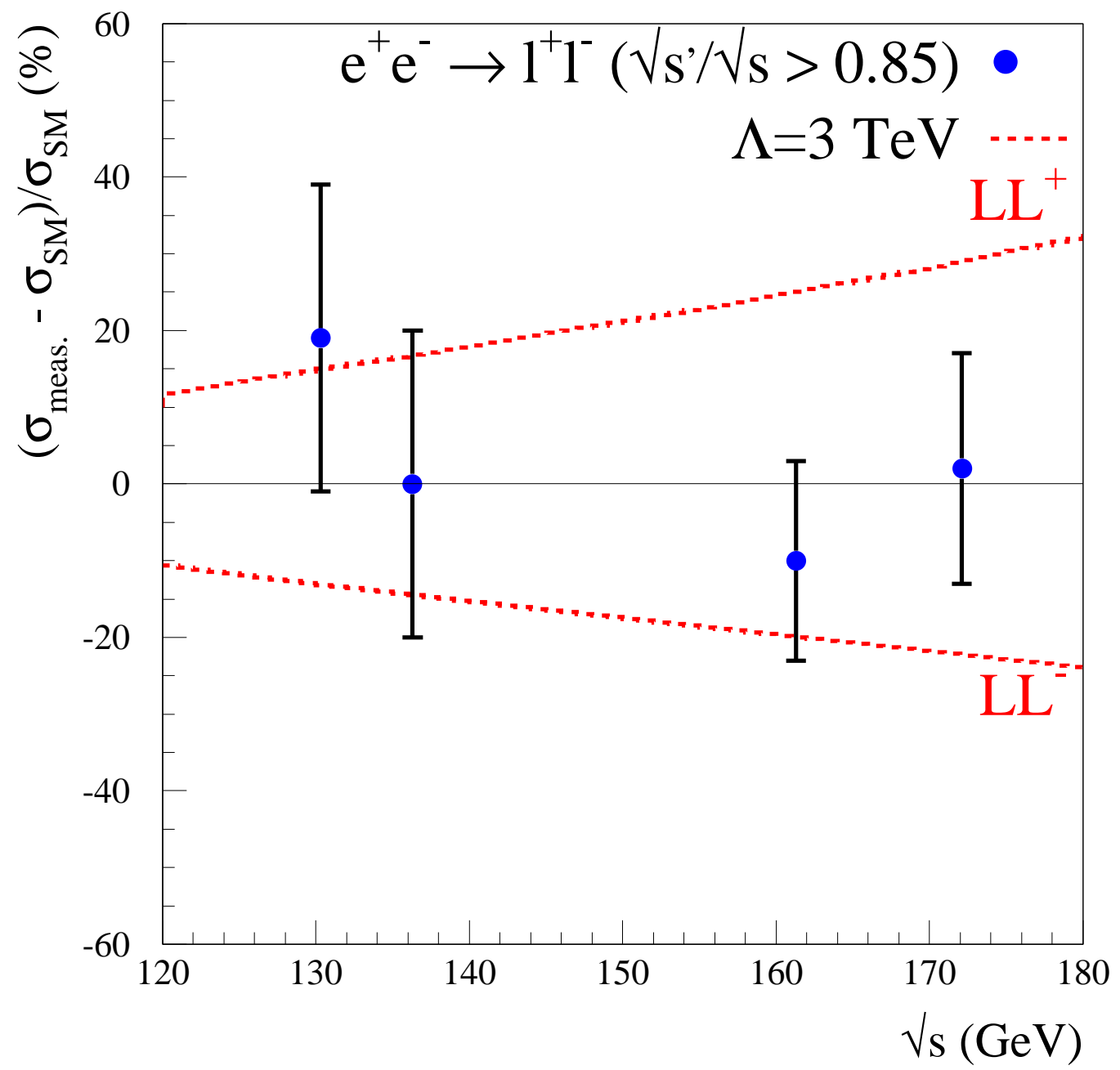

Figure 12: The expected deviations from the Standard Model for the LL \pm models compared to the weighted average of the deviations found in the combined data for $e^{+} e^{-} \rightarrow \mu^{+} \mu^{-}$and $e^{+} e^{-} \rightarrow \tau^{+} \tau^{-}$.

\begin{tabular}{|c|c|c|}
\hline Coupling & $\begin{array}{c}m_{\tilde{\nu}}=100 \mathrm{GeV} / c^{2} \\
(95 \% \text { c.1. })\end{array}$ & $\begin{array}{c}m_{\tilde{\nu}}=200 \mathrm{GeV} / c^{2} \\
(95 \% \text { c. } 1 .)\end{array}$ \\
\hline \hline$\lambda\left(t\right.$-chann. $\tilde{\nu}_{\ell}$ in $\left.e^{+} e^{-} \rightarrow \mu^{+} \mu^{-}\right)$ & 0.52 & 0.76 \\
$\lambda\left(t\right.$-chann. $\tilde{\nu}_{\ell}$ in $\left.e^{+} e^{-} \rightarrow \tau^{+} \tau^{-}\right)$ & 0.55 & 0.79 \\
\hline
\end{tabular}

Table 18: Upper limits on the couplings $\lambda$ in $t$ channel sneutrino exchange in $e^{+} e^{-} \rightarrow \mu^{+} \mu^{-}$and $e^{+} e^{-} \rightarrow \tau^{+} \tau^{-}$. The couplings involved are given in the text. 


\section{DELPHI}
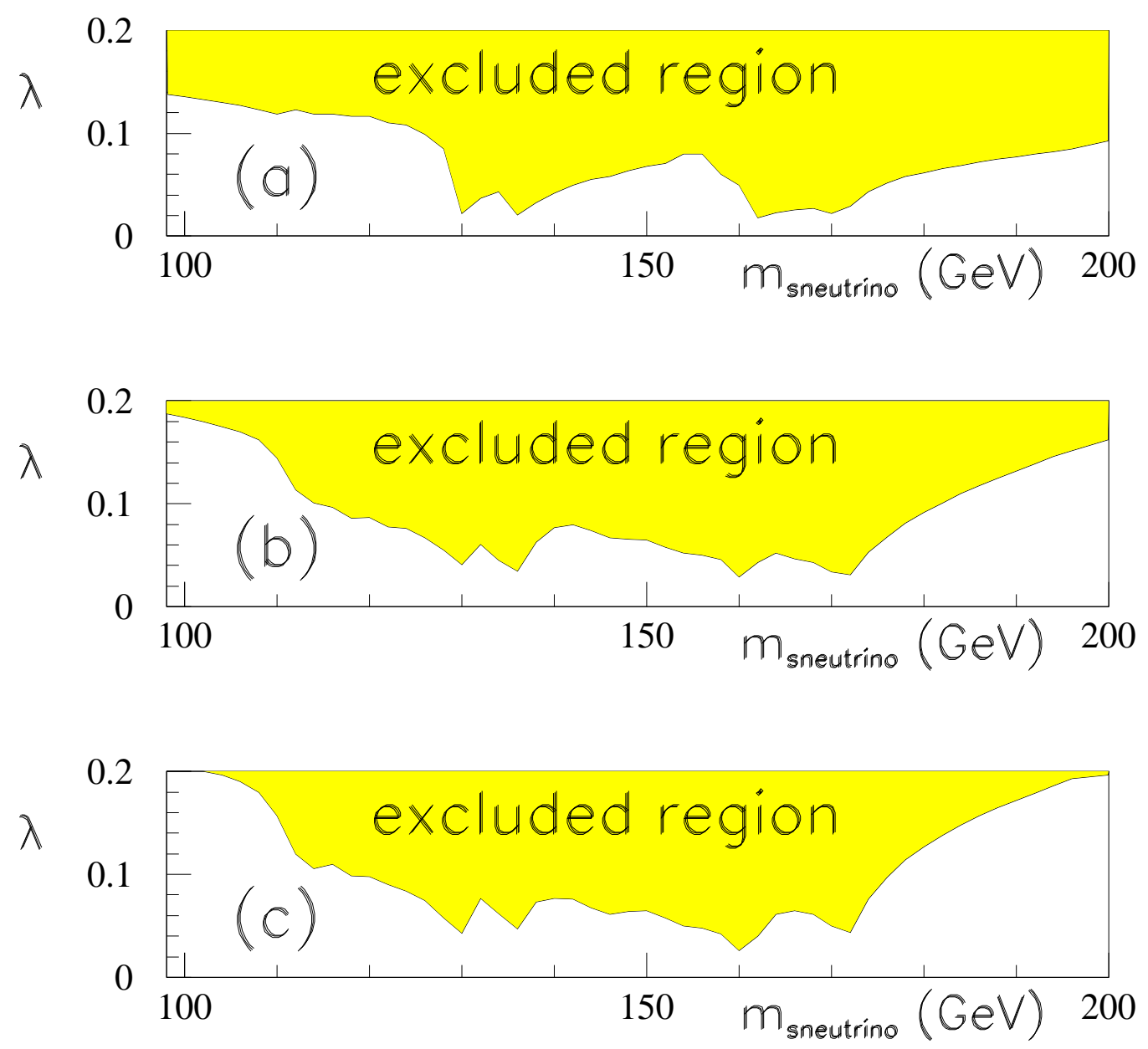

Figure 13: The $95 \%$ exclusion limits for (a) $\lambda_{121}$ ( or $\lambda_{131}$ ), as a function of $m_{\tilde{\nu}}$ obtained from the $e^{+} e^{-} \rightarrow e^{+} e^{-}$channel; (b) $\lambda_{131}=\lambda_{232}=\lambda$, as a function of $m_{\tilde{\nu}}$ obtained from the $e^{+} e^{-} \rightarrow \mu^{+} \mu^{-}$ channel; (c) $\lambda_{121}=\lambda_{233}=\lambda$, as a function of $m_{\tilde{\nu}}$ obtained from the $e^{+} e^{-} \rightarrow \tau^{+} \tau^{-}$channel. The sneutrino width is taken to be $1 \mathrm{GeV}$. It can be seen that the limits are better where the sneutrino mass is equal to the centre of mass energy of the data. 
where $\sigma_{q}$ denotes the measured quark cross-section and $\sigma^{S M}$ the expected cross-section in the Standard Model. The measured quark cross-section is defined as $\sigma_{q}=R_{q} \sigma_{h}$, where $R_{q}$ are the measured cross-section ratios given in Table 12 , and $\sigma_{h}$, given in Table 10 , is the average of the measured hadronic cross-sections for $\sqrt{\mathrm{s}^{\prime}} / \sqrt{\mathrm{s}}>0.85$ at centre-of-mass energies of 130-136 GeV and 161-172 GeV.

The results were compared with the model predictions for the cross-section ratio and asymmetry difference, for different values of the coupling constant, $\lambda_{\text {susy }}^{\prime}$. Upper and lower limits were evaluated from the values of $\lambda_{\text {susy }}^{\prime}$ consistent with the values of the measured quantities being within \pm 1.64 times the 1 standard deviation uncertainty from the measurements and from the Standard Model expectation, taking which ever gave the most conservative limit. Figure 14 shows the predictions and limits for down (up) type quarks in the final state taking a squark mass of $200 \mathrm{GeV} / c^{2}$, at a centre-of-mass energy of $166 \mathrm{GeV}$. The upper limits on $\lambda_{\text {susy }}^{\prime}$, derived from all the data analysed in this paper are given in table 19 for each quark flavour at $95 \%$ confidence, assuming a squark mass of $200 \mathrm{GeV} / \mathrm{c}^{2}$.

\subsubsection{Contact Interactions}

The cross-sections and asymmetries were predicted for final states with up and down type quarks, assuming contact interactions only couple one flavour of quark to electrons at a time.

The parameter $\Lambda$ of equation 6 was varied for the $L L, L R, R R$ and $R L$ models taking both $\eta=-1 / 2$ and $\eta=+1 / 2$ values, giving eight sets of predictions for the crosssections. From these predictions the cross-section ratios $\left(\sigma-\sigma^{S M}\right) / \sigma^{S M}$ were evaluated and compared to the upper limits on the measured cross-section ratios, as shown in Figure 15. The corresponding limits at $95 \%$ CL are also given in Table 20. The limits on $\Lambda$ are in the range from 1 to $4.4 \mathrm{TeV}$; the limits for bottom quarks are better than the limits for the down or strange quarks since the efficiency and purity of the bottom quark tag used is highest, leading to a smaller error on the bottom quark production cross-section.

\section{3 $\quad \mathrm{Z}^{\prime}$-bosons}

Fits were applied to data collected by DELPHI at LEP $1[1,2]$ as well as at LEP 2, i.e. at centre-of-mass energies of 88-94, 130, 136, 161 and $172 \mathrm{GeV}$. The observables used for the fits were the hadronic and leptonic cross-sections and the leptonic forward-backward asymmetries. Predictions for these observables within the $Z^{\prime}$ models $E_{6}$ and L-R and within a model independent approach were obtained using the program ZEFIT (5.0) [43] together with ZFITTER (5.0). Correlations between the data and errors of the LEP beam energy were taken into account as described in $[1,2]$.

\subsubsection{Model dependent fits}

The program ZEFIT provides predictions for the cross-sections and forward-backward asymmetries for each model as a function of the masses $M_{Z}, M_{H}$ and $M_{t}$, of the strong coupling constant $\left(\alpha_{s}\right)$, the mass of the $Z^{\prime}$-boson $\left(M_{\mathrm{Z}^{\prime}}\right)$, its mixing with the Z-boson $\left(\theta_{Z Z^{\prime}}\right)$, and of the $Z^{\prime}$-model parameters $\Theta_{6}$ (in case of $E_{6}$-models) or $\alpha_{L R}$ (in case of the L-R-model). In order to reduce the number of free parameters, the top mass was fixed to $M_{t}=175 \mathrm{GeV} / c^{2}$ and the coupling $\alpha_{s}$ to the value 0.123 . The mass of the Higgs was set to $M_{H}=300 \mathrm{GeV} / c^{2}$. Varying the values of these parameters has a negligible influence on the fit results. As the standard Z-boson mass would change in the presence 


\section{DELPHI limits on new Interactions}
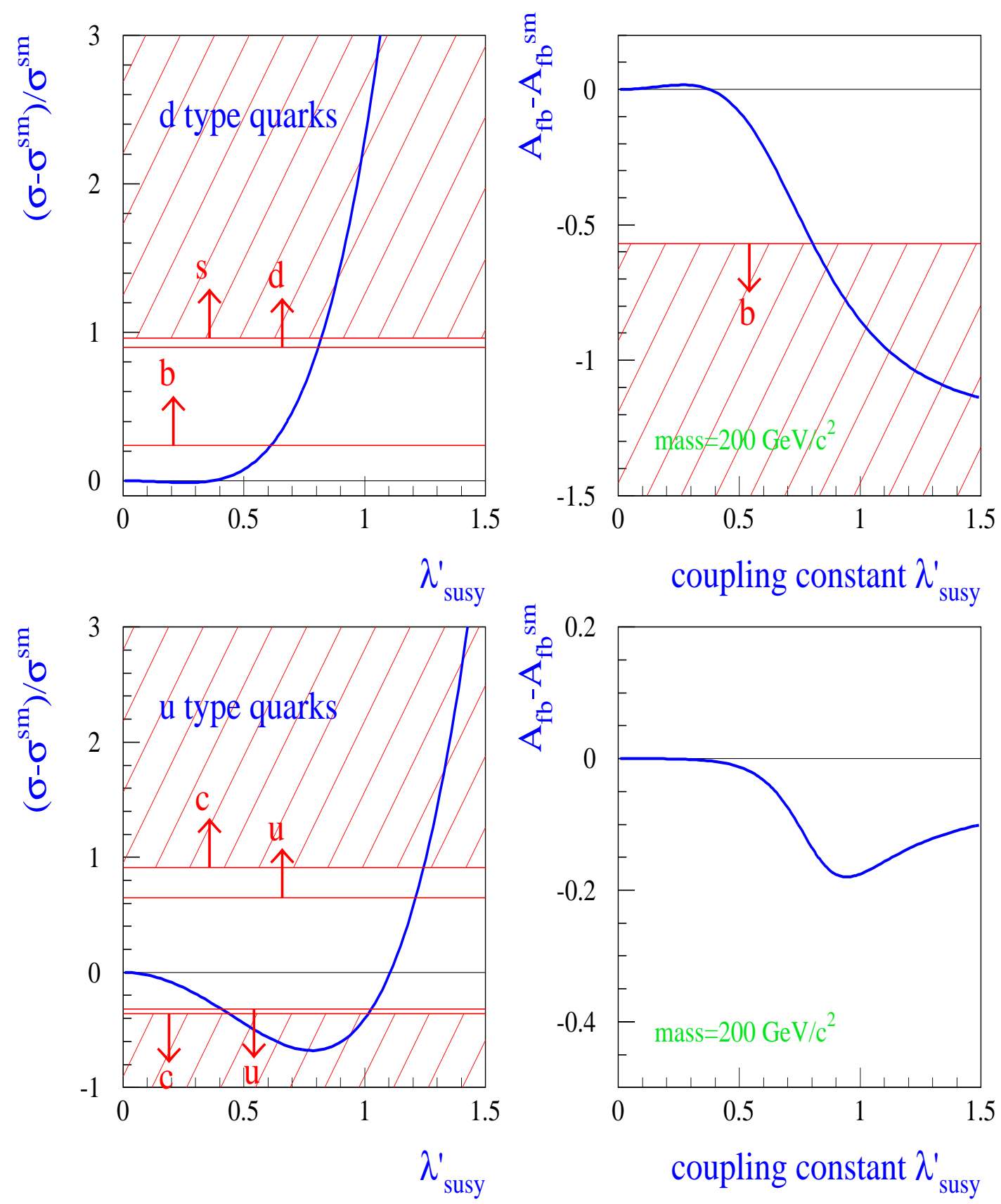

Figure 14: The curves show the expected cross-section ratio and asymmetry difference as a function of the coupling constant $\lambda_{\text {susy }}^{\prime}$ for down (upper plot) and up (lower plot) type quarks, assuming a squark with a mass of $200 \mathrm{GeV} / \mathrm{c}^{2}$ for processes (i) to (iii) as defined in section 5.3. The horizontal lines and the hatched area correspond to the different exclusion limits at $95 \%$ C.L. The arrow points to the excluded zone. The precision on $A_{\mathrm{FB}}$ for up type quarks does not allow a $95 \%$ C.L. limit to be placed on $\lambda_{s u s y}^{\prime}$ 


\begin{tabular}{|c|c|c|c|c|c|}
\hline Flavour & $\mathrm{d}$ & $\mathrm{s}$ & $\mathrm{b}$ & $\mathrm{u}$ & $\mathrm{c}$ \\
\hline \hline$\lambda_{\text {susy }}^{\prime}$ & 0.81 & 0.82 & 0.61 & 0.42 & 0.43 \\
\hline
\end{tabular}

Table 19: Lower limits at 95\% CL on the coupling constant $\lambda_{\text {susy }}^{\prime}$, in a supersymmetric scenario (see text) with a squark mass of $200 \mathrm{GeV} / c^{2}$ for down or strange quarks, bottom quarks, and up and charm quarks.

\begin{tabular}{|c|c|c|c|c|c|c|c|c|}
\hline Model & $\begin{array}{c}\mathrm{d} \text { or s } \\
\Lambda^{+}(\mathrm{TeV})\end{array}$ & $\begin{array}{c}\mathrm{b} \\
\Lambda^{+}\end{array}$ & $\begin{array}{c}\mathrm{u} \\
\Lambda^{+}\end{array}$ & $\begin{array}{c}\mathrm{c} \\
\Lambda^{+}\end{array}$ & $\begin{array}{c}\mathrm{d} \text { or s } \\
\Lambda^{-}(\mathrm{TeV})\end{array}$ & $\begin{array}{c}\mathrm{b} \\
\Lambda^{-}\end{array}$ & $\begin{array}{c}\mathrm{c} \\
\Lambda^{-}\end{array}$ \\
\hline \hline$L L$ & 2.4 & 4.4 & 1.0 & 1.0 & 2.8 & 3.9 & 2.4 & 2.1 \\
\hline$L R$ & 1.7 & 2.5 & 1.5 & 1.3 & 1.5 & 2.0 & 1.8 & 1.6 \\
\hline$R R$ & 1.9 & 3.1 & 1.2 & 1.1 & 1.4 & 1.7 & 2.1 & 1.9 \\
\hline$R L$ & 1.4 & 1.7 & 1.6 & 1.5 & 1.9 & 3.0 & 1.6 & 1.5 \\
\hline
\end{tabular}

Table 20: Lower limits at 95\% CL on the energy scale $\Lambda$ for different forms of the interaction for down or strange quarks, bottom, up and charm quarks. 


\section{DELPHI limits on contact Interactions}
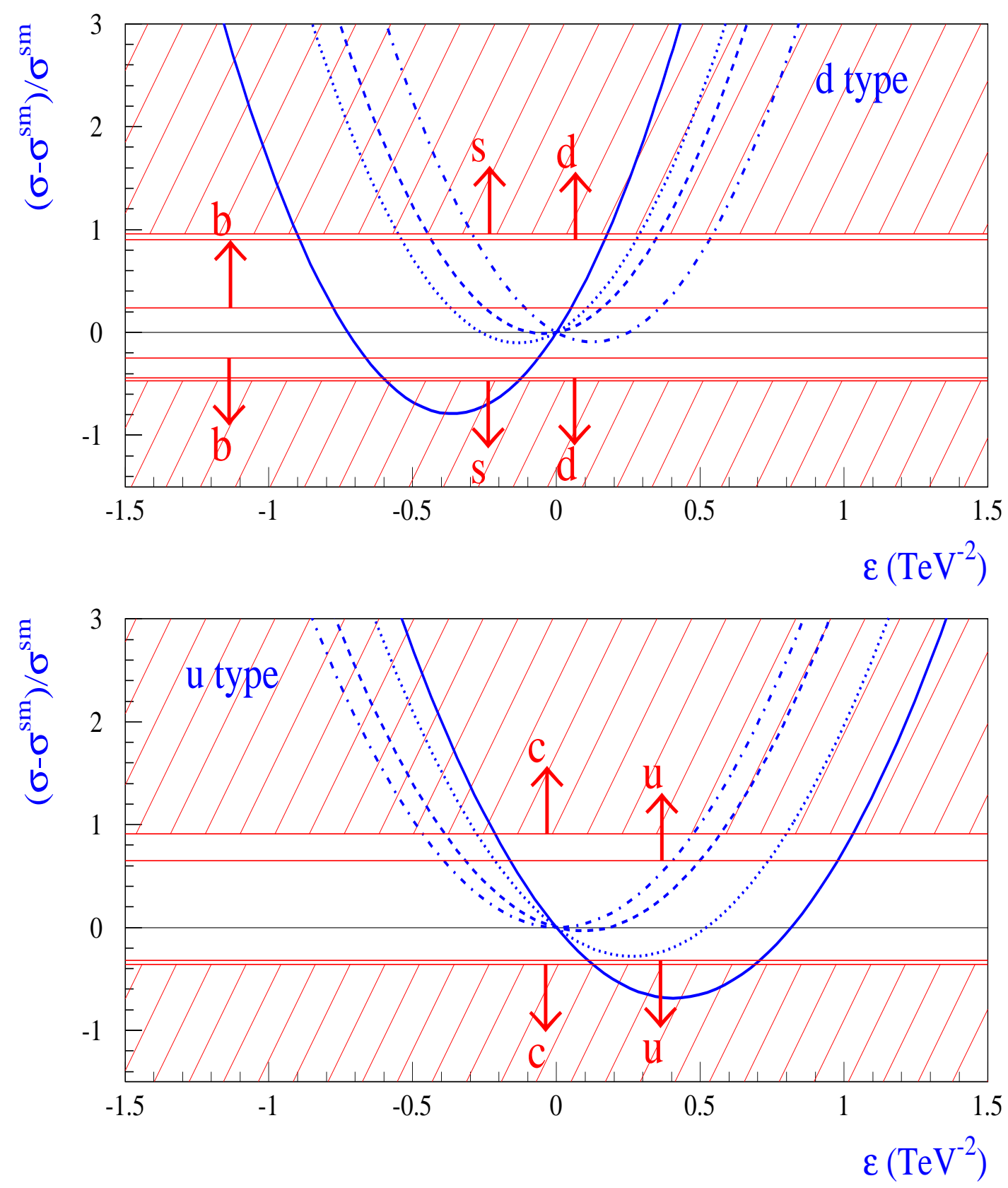

Figure 15: The curves show the expected cross-section ratio as a function of $\epsilon\left(=1 / \Lambda^{2}\right)$ for down (upper plot) and up (lower plot) type quarks, assuming a new interaction of the form Eq. 6 . The solid curve corresponds to a $L L+(-)$, the dotted to a $L R+(-)$, the dashed to a $R R+(-)$ and the dotted-dashed to a $R L+(-)$ interaction. Negative values for $\epsilon$ correspond to negative $\eta$ values. The horizontal lines and the hatched area correspond to the different exclusion limits at $95 \% \mathrm{CL}$. The arrow points to the excluded zone. 
of a non-zero mixing between $\mathrm{Z}$ and $\mathrm{Z}^{\prime}, M_{Z}$ was left free to vary together with $M_{\mathrm{Z}^{\prime}}$ and $\theta_{Z Z^{\prime}}$. The $E_{6}$ inspired models $\chi, \psi$ and $\eta$ were considered. In case of the L-R model, $\alpha_{L R}$ was set to 1.1 .

A $\chi^{2}$ was formed by comparing the measured observables to their predicted values. The fitted Z-mass was found to be compatible with its standard value. No evidence was found for the existence of a $Z^{\prime}$-boson in any of the models. The $95 \%$ confidence level limits on $M_{Z^{\prime}}$ and $\theta_{Z Z^{\prime}}$ were computed for each model by determining the contours of the domain in the $M_{Z^{\prime}}-\theta_{Z Z^{\prime}}$ plane where $\chi^{2}<\chi_{\min }^{2}+5.99$. The allowed regions for $M_{Z^{\prime}}$ and $\theta_{Z Z^{\prime}}$ are shown in figure 16 . The lower limit of the $Z^{\prime}$ mass varies between $200 \mathrm{GeV} / c^{2}$ and $280 \mathrm{GeV} / \mathrm{c}^{2}$, depending on the model considered. The limits obtained are given in Table 21.

These results improve substantially the limits from a previous publication of the DELPHI collaboration, based on measurements performed on and below the $Z$ peak [44]. The limits on the $Z^{\prime}$ mass are weaker than those from direct searches at the TEVATRON [45], where values of the order of $600 \mathrm{GeV} / c^{2}$ were obtained. A substantial improvement of the limits provided by LEP is expected from data taking at energies exceeding $180 \mathrm{GeV}$.

\subsubsection{Model independent fits}

The program ZEFIT was used to predict cross-sections and forward-backward asymmetries as a function of the $Z^{\prime}$-mass and of the couplings $a_{f}^{\prime}$ and $v_{f}^{\prime}$. In the most general case, all couplings $a_{f}^{\prime}$ and $v_{f}^{\prime}$ should be treated as free parameters. The number of parameters can be reduced if lepton universality is assumed. Bounds on the couplings $a_{l}^{\prime}$ and $v_{l}^{\prime}$ can then be obtained from fits to observables describing leptonic final states. Measurements from an $e^{+} e^{-}$collider are the most direct input for such an analysis, as no assumptions about the couplings of the $Z^{\prime}$ to quarks have to be made.

Fits were performed to the leptonic cross-sections and forward-backward asymmetries. Several values of the mass of the $Z^{\prime}$ were considered (i.e. 300,500 and $1000 \mathrm{GeV} / \mathrm{c}^{2}$ ), and the ZZ'-mixing was neglected. Figure 17 shows the values of the couplings $a_{f}^{\prime}$ and $v_{f}^{\prime}$ which are compatible with the DELPHI data with a confidence level of $95 \%$. The limits on the normalised couplings are $\left|a_{l^{\prime}}^{N}\right|<0.19$ and $\left|v_{l^{\prime}}^{N}\right|<0.44$.

\section{Summary and conclusions}

The data collected with the DELPHI detector at high energies (130-172 GeV) during LEP operation in 1995 and 1996, have been used to determine the hadronic and leptonic cross-sections and leptonic forward-backward asymmetries. In addition, measurements of the cross-section ratios and forward-backward asymmetries for flavour-tagged samples of light (uds), $c$ and b quarks have been made.

The results of the hadronic and leptonic cross-sections and leptonic forward-backward asymmetries, together with data collected previously at the energies near the $Z^{0}$ resonance peak $(88-93 \mathrm{GeV})$, have been interpreted by performing fits using the S-matrix formalism. The precision of the determination of the parameters of this ansatz has been considerably improved.

The DELPHI data on $e^{+} e^{-} \rightarrow f \bar{f}$ collected above the $Z$ resonance have been analysed in terms of models beyond the Standard Model. The first set included possible contact interactions between leptons. No evidence was found for such interactions and $95 \%$ confidence lower limits were placed on the energy scale, $\Lambda$ in such models, with values of the order of a few $\mathrm{TeV}$. In the second set of models, the exchange of a sneutrino in 


\section{DELPHI}
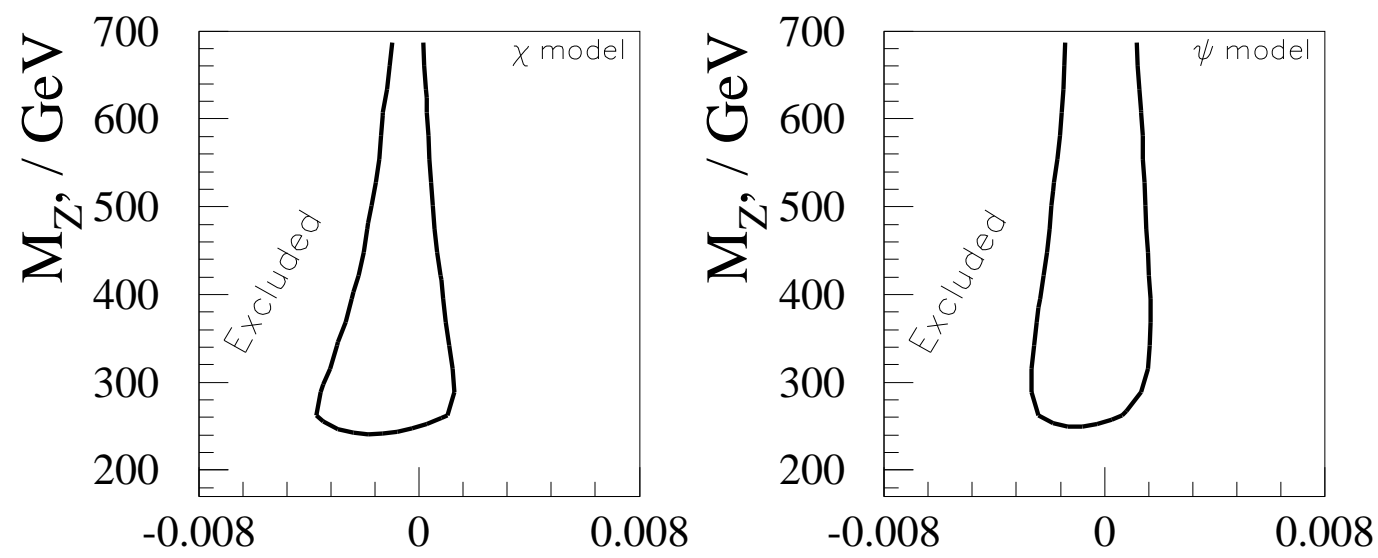

$\Theta_{\mathrm{ZZ}}$
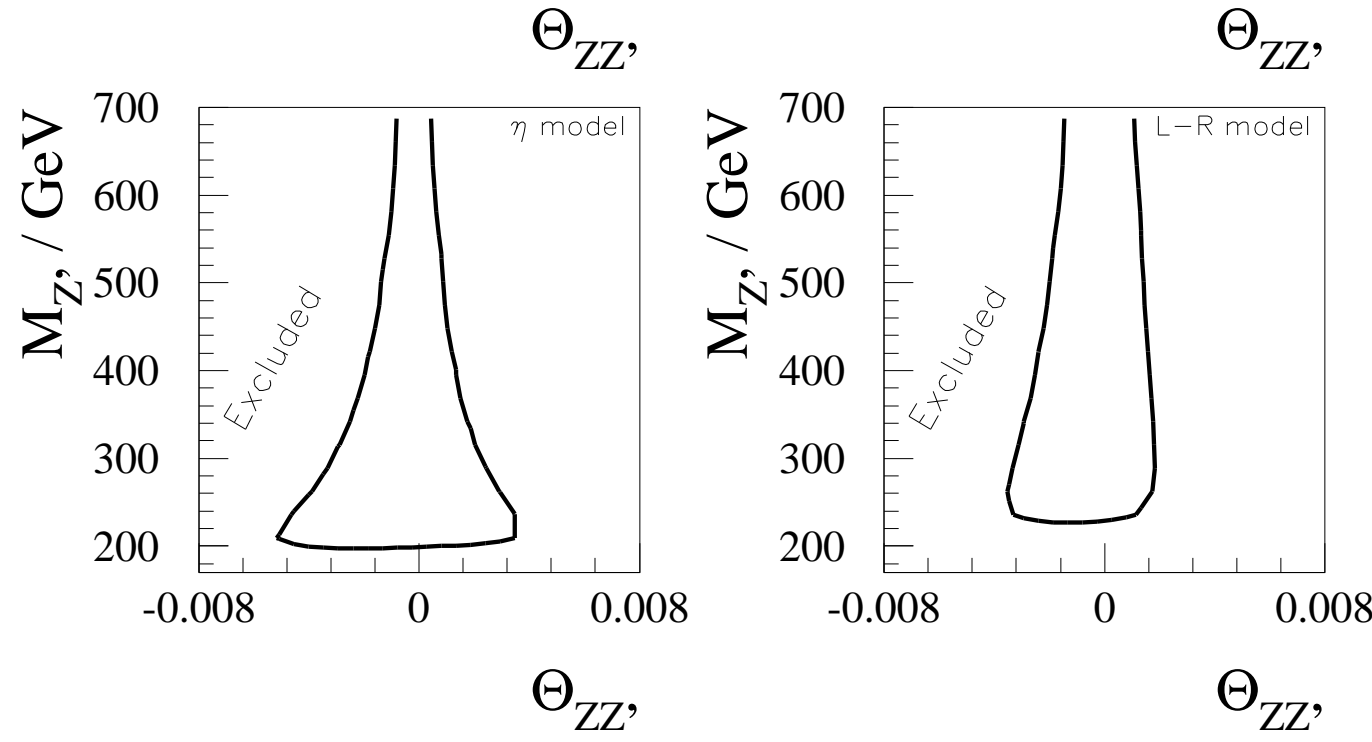

Figure 16: The allowed domain in the $M_{\mathrm{Z}^{\prime}}-\theta_{Z \mathrm{Z}^{\prime}}$ plane for the $\chi, \psi, \eta$ and $\mathrm{L}-\mathrm{R}$ models. The contours show the $95 \%$ confidence level limits.

\begin{tabular}{|c|c|c|c|c|}
\hline Model & $\chi$ & $\psi$ & $\eta$ & L-R \\
\hline \hline$M_{\mathrm{Z}^{\prime}}^{\text {limit }}\left(\mathrm{GeV} / c^{2}\right)$ & 250 & 280 & 200 & 230 \\
\hline$\left|\theta_{Z \mathrm{Z}^{\prime}}^{\text {limit }}\right|$ & 0.0033 & 0.0021 & 0.0046 & 0.0031 \\
\hline
\end{tabular}

Table 21: $95 \%$ confidence level lower limits on the $\mathrm{Z}^{\prime}$ mass and upper limits on the $\mathrm{ZZ}^{\prime}$ mixing angle within the $\chi, \psi, \eta$ and L-R models. 


\section{DELPHI}
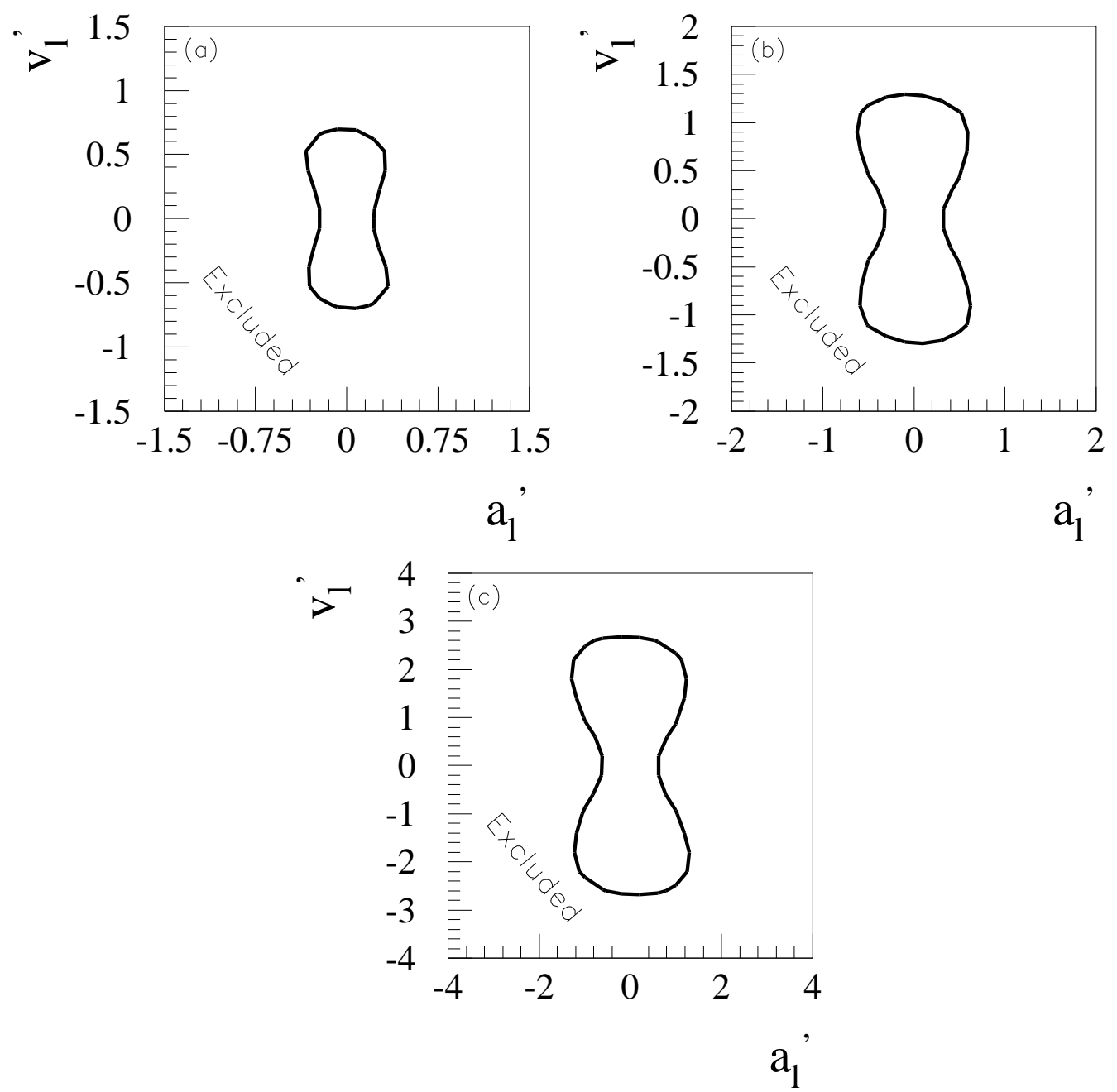

Figure 17: $95 \%$ confidence level limits on the couplings $a_{l}^{\prime}$ and $v_{l}^{\prime}$ of the $\mathrm{Z}^{\prime}$ to leptons. The values of the $\mathrm{Z}^{\prime}$ mass considered are (a) 300 , (b) 500 and (c) $1000 \mathrm{GeV} / c^{2}$. 
either the $s$ or $t$ channel, as a manifestation of R-parity violating supersymmetry, was considered. Again, no evidence for such effects was found and limits were placed on the coupling constants between the sneutrino and charged leptons, the values being sensitive to the model assumed.

The results of the cross-section ratios and forward-backward asymmetries for tagged samples of different quark flavours are in agreement with the expectations of the Standard Model. The data were interpreted in terms of possible new interactions. First, interactions with R-Parity violating supersymmetry were considered and exclusion limits were derived. Secondly, more general contact interactions were considered and limits on the mass scales in the range from 1.7 to $7.9 \mathrm{TeV}$ were obtained for different quark flavours.

Finally, the existence of an additional neutral massive gauge boson, $\mathrm{Z}^{\prime}$ was investigated. No evidence for a $Z^{\prime}$ was found within the framework of $E_{6}$ and L-R models. Limits on the $Z^{\prime}$-mass and on its mixing angle with the $Z$ were derived, which improve substantially former limits obtained by the DELPHI collaboration. Overall, $M_{\mathrm{Z}^{\prime}}$ was found to be greater than $200 \mathrm{GeV}$ for all the models considered. A model independent fit was also performed in order to derive limits on the couplings of a possible $Z^{\prime}$ to leptons. The $95 \%$ confidence level upper bounds obtained for the normalised couplings of a $Z^{\prime}$ are $\left|a_{l^{\prime}}^{N}\right|<0.19$ and $\left|v_{l^{\prime}}^{N}\right|<0.44$.

\section{Acknowledgements}

We would like to thank H. Dreiner, G. Bhattacharyya, H. Spiesberger and P.M. Zerwas for very helpful discussions on R-Parity violating SUSY effects. We would also like to thank Sabine Riemann for providing us with the program ZEFIT and for many helpful discussions concerning its use.

We are greatly indebted to our technical collaborators, to the members of the CERNSL Division for the excellent performance of the LEP collider, and to the funding agencies for their support in building and operating the DELPHI detector.

We acknowledge in particular the support of:

Austrian Federal Ministry of Science and Traffics, GZ 616.364/2-III/2a/98, FNRS-FWO, Belgium,

FINEP, CNPq, CAPES, FUJB and FAPERJ, Brazil,

Czech Ministry of Industry and Trade, GA CR 202/96/0450 and GA AVCR A1010521,

Danish Natural Research Council,

Commission of the European Communities (DG XII),

Direction des Sciences de la Matière, CEA, France,

Bundesministerium für Bildung, Wissenschaft, Forschung und Technologie, Germany,

General Secretariat for Research and Technology, Greece,

National Science Foundation (NWO) and Foundation for Research on Matter (FOM), The Netherlands,

Norwegian Research Council,

State Committee for Scientific Research, Poland, 2P03B06015, 2P03B03311 and $\mathrm{SPUB} / \mathrm{P} 03 / 178 / 98$,

JNICT-Junta Nacional de Investigação Científica e Tecnológica, Portugal,

Vedecka grantova agentura MS SR, Slovakia, Nr. 95/5195/134,

Ministry of Science and Technology of the Republic of Slovenia,

CICYT, Spain, AEN96-1661 and AEN96-1681,

The Swedish Natural Science Research Council,

Particle Physics and Astronomy Research Council, UK,

Department of Energy, USA, DE-FG02-94ER40817. 


\section{References}

[1] DELPHI Collaboration, P. Abreu et al. , Nucl. Phys. B417 (1994) 3.

[2] DELPHI Collaboration, P. Abreu et al. , Nucl. Phys. B418 (1994) 403.

DELPHI Collaboration, in preparation.

[3] DELPHI Collaboration, P. Aarnio et al. , Nucl. Instr. \& Meth. A303 (1991) 233.

[4] DELPHI Collaboration, P. Abreu et al. , Nucl. Instr. \& Meth. A378 (1996) 57.

[5] C. Adloff et al. (H1 Collaboration), Z. Phys. C74 (1997) 191;

J. Breitweg et al. (Zeus Collaboration), Z. Phys. C74 (1997) 207.

[6] J. Kalinowski et al. , Z. Phys. C74 (1997) 595.

[7] G. Altarelli, Nucl. Instr. \& Meth. B506 (1997) 3;

D. Choudhury, Phys Lett. B376 (1996) 201.

[8] ALEPH Collaboration, D. Buskulic et al. , Phys. Lett. B378 (1996) 373

ALEPH Collaboration, R. Barate et al. , Phys. Lett. B399 (1997) 329.

[9] L3 Collaboration, M. Acciarri et al., Phys. Lett. B407 (1997) 361.

L3 Collaboration, M. Acciarri et al. , Phys. Lett. B370 (1996) 195.

[10] OPAL Collaboration; K. Ackerstaff et al., Euro. Phys. J. C2 (1998) 441.

[11] "The LEP Energy Calibration in 1996", LEP Energy Group/97-01.

[12] "Energies for LEP 1.5 run", LEP Energy Group/97-01.

[13] A. C. Benvenuti et al. , "The DELPHI Small Angle Tile Calorimeter", contribution to IEEE NSS 1994

[14] S. Jadach, O Nicrosini et al. , "Event Generators for Bhabha Scattering", CERN Yellow Report 96-01, vol 2, p229.

[15] T.Sjöstrand, CERN-TH/92-6488,

T. Sjöstrand, PYTHIA 5.7 and JETSET 7.4, CERN-TH 7112/93,

T. Sjöstrand and M. Bengtsson, Comput. Phys. Commun. 43(1987)367,

T. Sjöstrand, Comput. Phys. Commun. 39(1986)347.

[16] S. Jadach, B.F.L. Ward, Z. Was, Comp. Phys. Comm. 79 (1994) 503.

[17] J.E. Campagne and R. Zitoun, Zeit. Phys. C43 (1989) 469.

[18] D. Bardin et al., "ZFITTER: An Analytical Program for Fermion Pair Production in $e^{+} e^{-}$Annihilation", preprint CERN-TH 6443 (1992) and references therein.

[19] DELPHI Collaboration, P. Abreu et al. , Zeit. Phys. C73 (1996) 11.

[20] S. Nova, A. Olchevski and T. Todorov, DELPHI Note 90-35 PROG/152, unpublished.

[21] F.A. Berends, W. Hollik and R. Kleiss, Nucl. Phys. B304 (1988) 712.

[22] DELPHI Collaboration, P. Abreu et al. , Zeit. Phys. C70 (1996) 531;

G. Borisov and C. Mariotti, "Performance of b-tagging at LEP2", DAPNIA/SPP

Report 97-06, INFN/ISS Report 97-03.

G. Borisov and C. Mariotti, NIM A372 (1996) 181.

[23] DELPHI Collaboration, P. Abreu et al. , Phys. Lett. B322 (1994) 459.

[24] G. Montagna et al. , Nucl. Phys. B401 (1993) 3, and Comput. Phys. Commun. 76 (1993) 328.

[25] DELPHI Collaboration, P. Abreu et al. , Z. Phys. C75 (1997) 581.

[26] W. Beenakker et al. , Nucl. Phys. B349 (1991) 323.

[27] A. Leike and S. Riemann, Phys. Lett. B273 (1991) 513.

[28] S. Riemann, Phys. Lett. B293 (1992) 451.

[29] E. Eichten, K. Lane, M. Peskin, Phys. Rev. Lett. 50 (1983) 811.

[30] H. Kroha, Phys. Rev. D46 (1992) 58.

[31] ALEPH Collaboration, D. Buskulic et al. , Zeit. Phys. C59 (1993) 215. 
[32] OPAL Collaboration, G. Alexander et al., Phys. Lett. B 387 (1996) 432.

[33] J. Kalinowski et al. , DESY 97-044 [hep-hp/9703436].

[34] H. Dreiner, HEP/PH-9707435 (1997) and private communication.

[35] A. Leike, S. Riemann and T. Riemann, Munich University preprint LMU-91/6.

[36] A. Leike, S. Riemann and T. Riemann, preprint CERN-TH 6545/92.

[37] P. Langacker, R.W. Robinett and J.L. Rosner, Phys. Rev. D30 (1984) 1470;

D. London and J.L. Rosner, Phys. Rev. D34 (1986) 1530.

[38] J.C. Pati and A. Salam, Phys. Rev. D10 (1974) 275;

R.N. Mohapatra and J.C. Pati, Phys. Rev. D11 (1975) 566.

[39] A. Leike, Zeit. Phys. C62 (1994) 265.

[40] G. Altarelli et al. (eds), "Z Physics at LEP I", CERN 89-08;

G. Altarelli et al. (eds), "Physics at LEP II", CERN 96-01.

[41] DELPHI Collaboration, P. Abreu et al. , Zeit. Phys. C72 (1996) 31;

ALEPH Collaboration, R. Barate et al. , Phys. Lett. B399 (1997) 329.

[42] M. Martinez and R. Miquel, Zeit. Phys. C53 (1992) 115.

[43] S. Riemann, FORTRAN program ZEFIT, version 5.0.

[44] DELPHI Collaboration, Zeit. Phys. C65 (1995) 603.

[45] CDF Collaboration, F. Abe at al, Phys. Rev. Lett.79 (1997) 2192. 